Supporting U.S. Industry, Government, and the Scientific Community

by providing measurement services and research for electronic,

optical, and radiation technology.
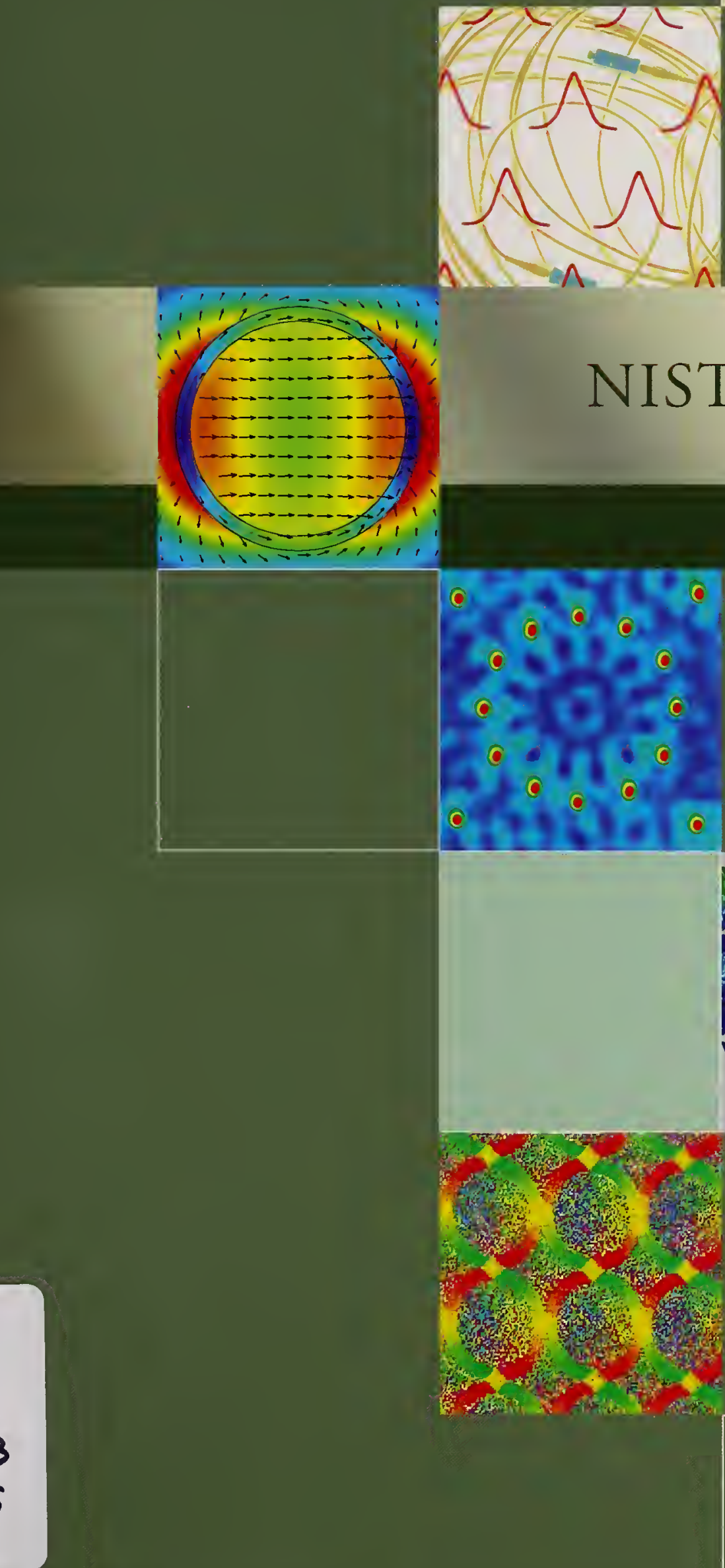

\title{
NIST Physics Laboratory
}

\section{QC 100 .457 $\pm 1033$ 2005 C. 2}
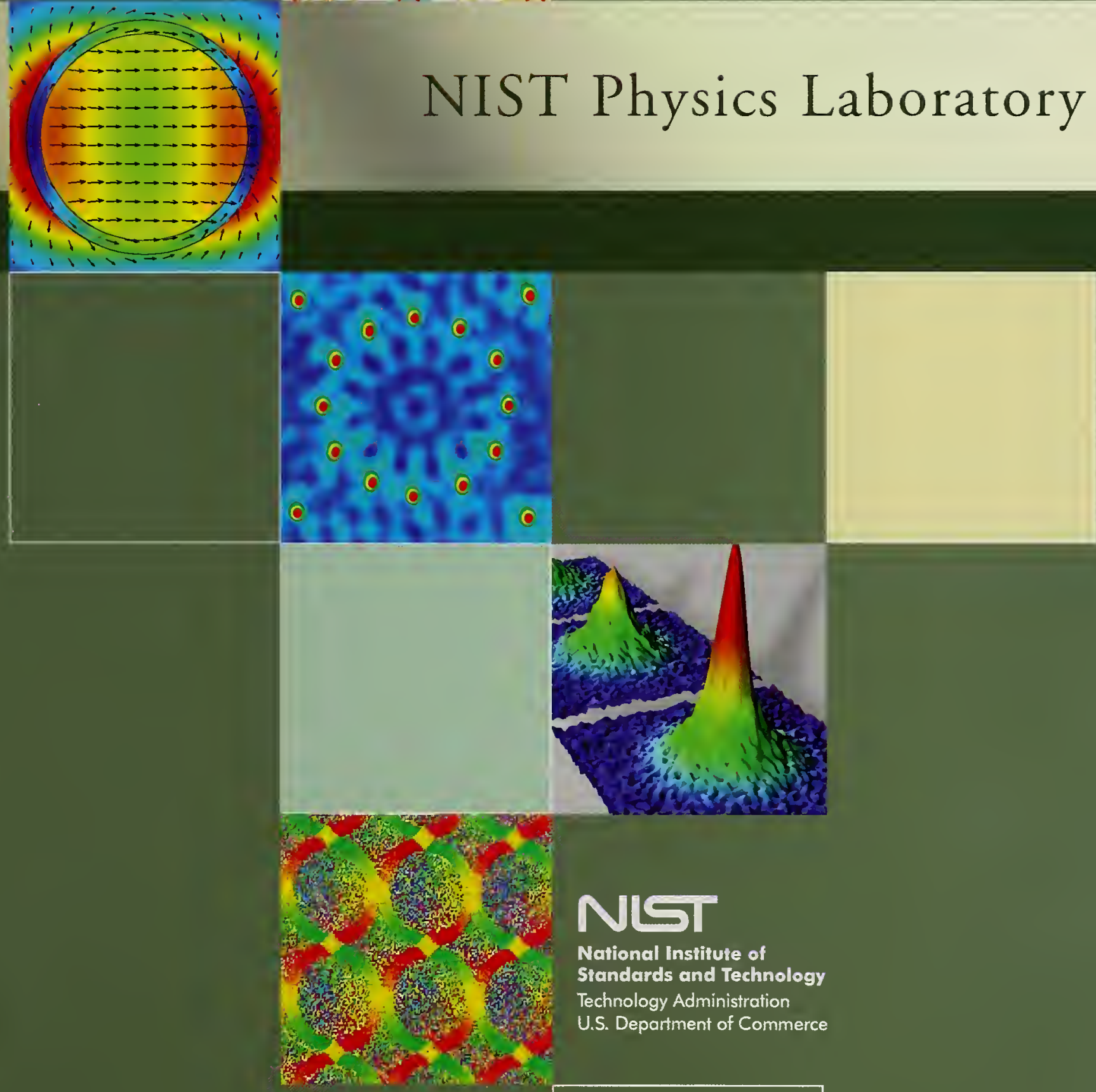

\section{NGT}

\section{National Institute of}

Standards and Technology

Technology Administration

U.S. Department of Commerce

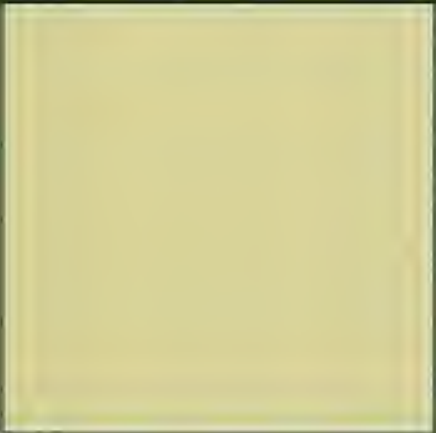


Physics Laboratory at a Glance

Director's Message

Optical Technology Division

Office of Electronic Commerce in

Scientific and Engineering Data
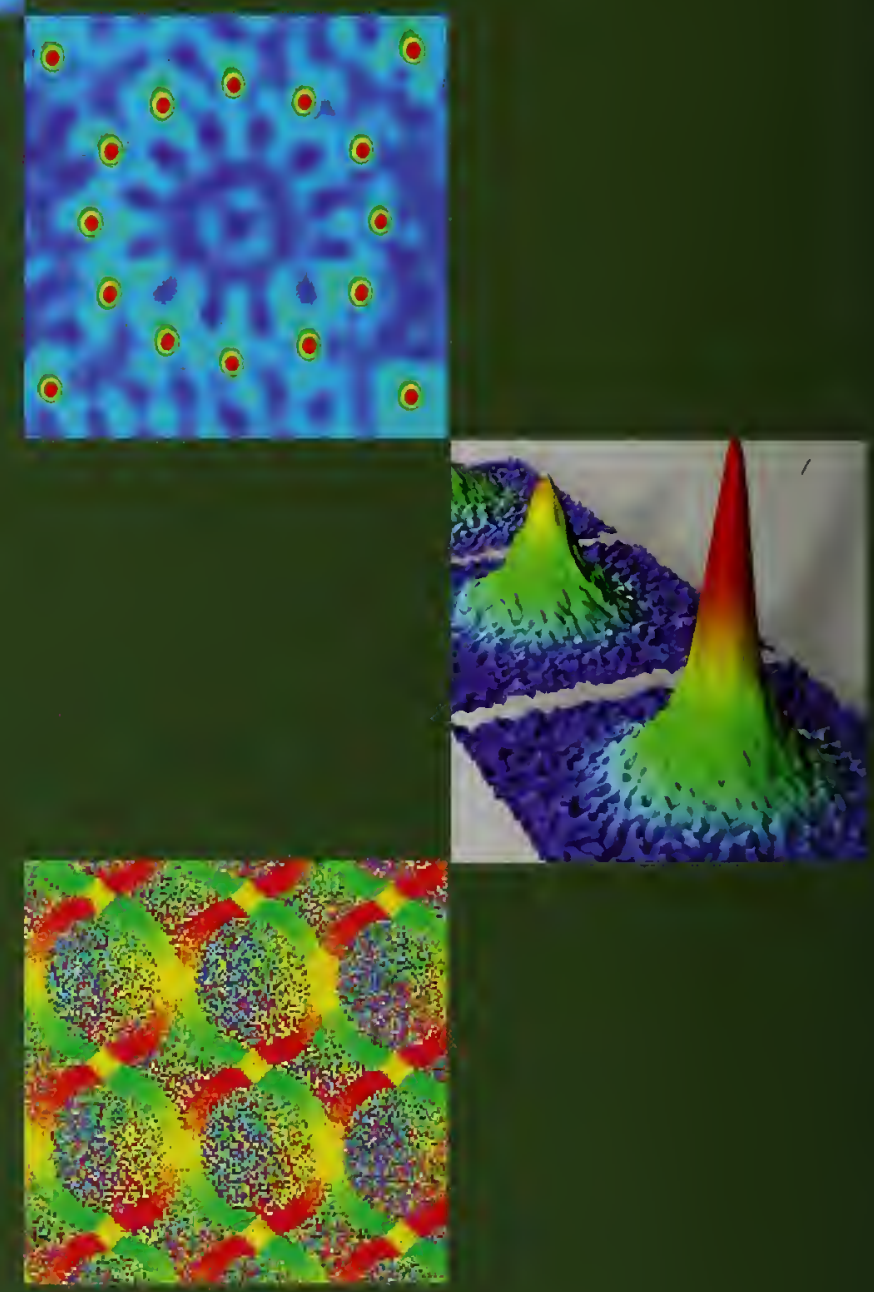


\section{PL Vision}

Preeminent performance in measurement science, technology, and services.

\section{PL Mission}

The mission of the NIST Physics Laboratory is to support U.S. industry, government, and the scientific community by providing measurement services and research for electronic, optical, and radiation technology. The Laboratory provides the foundation for metrology of optical and ionizing radiation, time and frequency, and fundamental quantum processes.

\section{PL Goals}

The Physics Laboratory develops goals within its mission and focus areas. These include health care quality assurance, nanoscale measurements and data, and measurements and standards that support homeland security. In addition to these over-arching focus areas, which are shared by other NIST Laboratories, PL has substantial programmatic interest in time and frequency, optical and photonic, and environmental and energy metrology and applications.

\section{Electron and Optical Physics}

Division: to support emerging electronic, optical, and nanoscale technologies.

Atomic Physics Division: to determine atomic properties and investigate fundamental quantum interactions.

Optical Technology Division: to provide the foundation for optical radiation measurements for our Nation.

Ionizing Radiation Division: to provide the foundation of ionizing radiation measurements for our Nation.

Time and Frequency Division: to provide the foundation of frequency measurements and civil timekeeping for our Nation.
Quantum Physics Division: to make transformational advances at the frontiers of measurement science, in partnership with the University of Colorado at JILA.

Office of Electronic Commerce in Scientific and Engineering Data: to coordinate and facilitate the electronic dissemination of technical information via the Internet.

\section{PL Resources}

192 full-time staff ( 140 scientific) with expertise in:

- Atomic, molecular, and optical physics

- Computational physics

- Condensed matter physics

- Health physics

- Medical physics

- Nuclear physics

- Biophysics

- Chemistry

- Metrology and precision measurement

\section{$\$ 67$ million annual budget}

Unique facilities, including:

- Bidirectional Optical Scattering Facility

- Center for High-Accuracy

Retroreflection Measurements

(CHARM)

- Electron Beam Ion Trap (EBIT)

- Electron Paramagnetic

Resonance Facility

- EUV Optics Fabrication and Characterization Facility

- High-Accuracy Cryogenic Radiometry

- High-Illuminance Standards and Calibration Facility

- High-Resolution UV and Optical Spectroscopy Facility

- W.M. Keck Optical Measurement Laboratory

- Low-Background Infrared Radiation Facility (LBIR)

- Magnetic Microstructure Measurement Facility

- Mammographic X-Ray Instrument Calibration Range
- Medical-Industrial Radiation Facility (MIRF)

- Nanoscale Physics Laboratory

- Neutron Imaging Facility

- Neutron Interferometer and Optics Facility (NIOF)

- Radiation Detector Test Facility

- Radiopharmaceutical Standardization Laboratory

- Spectral Irradiance and Radiance Calibrations with Uniform Sources Facility (SIRCUS)

- Synchrotron Ultraviolet Radiation Facility (SURF III)

\section{Standard time dissemination} services:

- Radio stations WWV, WWVH, and WWVB

- Automated Computer Time Service (ACTS)

- Internet Time Service (ITS)

Measurement and calibration services for:

- Color and color temperature

- Dosimetry of x rays, gamma rays, and charged particles

- Neutron sources and neutron dosimetry

- Optical properties of materials

- Optical wavelength

- Oscillator frequency

- Phase and amplitude noise

- Photodiode spectral responsivity

- Photometry (e.g., luminous intensity, luminous flux, illuminance)

- Radiance temperature

- Radiation detectors

- Radioactivity sources

- Spectral radiance and irradiance

- Spectral transmittance and reflectance

\section{PL Website}

http://physics.nist.gov/ 


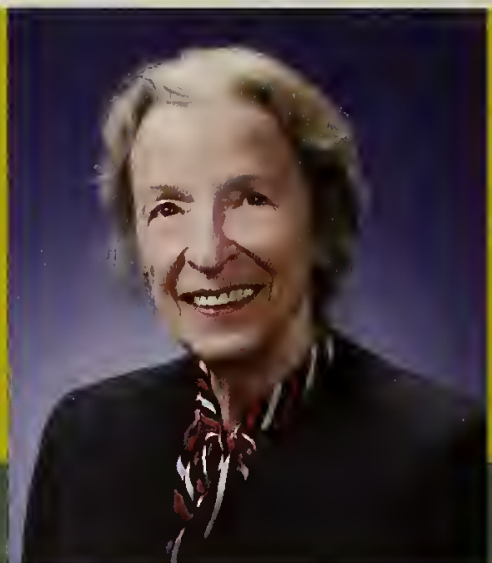

e hope this report conveys the excitement and the relevance of the programs within the NIST Physics Laboratory. First and foremost, we support U.S. industry by providing measurement services and research for optical, electronic, and radiation technologies. Our great strength-and what distinguishes us from an academic or industrial laboratory-is that we are vertically integrated. Our world-class measurement services are backed by state-of-the-art engineering efforts to develop new measurement standards, which are in turn supported by frontier, mission-oriented research that anticipates the Nation's future measurement needs.

Thus the Laboratory addresses the fundamental triad of standards, measurements, and data in a climate of vigorous and competitive research. We believe that the quality of our service stems in large measure from the breadth, vigor and excellence of our research programs and that our contributions gain credibility because they are based on the best technical judgment available.
For example, our Time and Frequency Division is delivering seven different kinds of time and frequency services while also developing optical frequency atomic clocks, chip-scale atomic clocks, and a futuristic atomic clock for space flight. While our Internet Time Service provides official U.S. time in over a billion daily transactions, we are also pursuing research on trapped ions for the next generation of frequency standards - and quantum-logic devices. Similarly, our Ionizing Radiation 
Division is developing highly sensitive neutron detectors for homeland security while also using ultracold neutrons to investigate symmetries and parameters of the nuclear weak interaction.

We maintain the U.S. national standards for the Système International (SI) base units of time (the second), light (the candela), and noncontact thermometry (the kelvin, especially above $1200 \mathrm{~K})$. We provide the basis for such SI derived units as the hertz (frequency), the becquerel (radioactivity), and the optical watt and the lumen (light output). At the same time, scientists in the Physics Laboratory work with industry to develop new measurement technologies that can be applied to such fields as communications, microelectronics, nanomagnetics, photonics, industrial radiation processing, the environment, health care, transportation, space, energy, security, and defense.

Our partners are many and our outreach is extensive. For optical radiation measurements, we rely heavily on the Council for Optical Radiation Measurements (CORM), formed to help define pressing problems and projected national needs in radiometry and photometry. Its aim is to establish a consensus on industrial and academic requirements for physical standards, calibration services, and interlaboratory collaborative programs in the fields of ultraviolet, visible, and infrared measurements. Similarly, the Council on Ionizing Radiation Measurements and Standards (CIRMS) helps to advance and disseminate the physical standards needed for the safe and effective application of ionizing radiation, including $\mathrm{x}$ rays, gamma rays, and energetic particles such as electrons, protons, and neutrons. When we can assist in an important area of measurement or research, we may form Cooperative Research and Development Agreements with industry groups or individual firms. Laboratory scientists serve with distinction in standards-development committees, and readily give of their time to assist the public.

We have been recognized for the quality and excellence of our programs and staff many times over the years, by the American Physical Society, the Optical Society of America, and other leading scientific organizations. Members of our staff have been elected to fellowship in the National Academy of Science, the American Association for the Advancement of Science, the American Association of Physicists in Medicine, and orher esteemed bodies. Twice since 1997, Laboratory scientists have won the Nobel Prize in Physics. Beginning on page 54 of this report, we highlight some of the awards and honors bestowed upon us recently.

Our talent is focused on meeting today's challenges-in biosystems and health care, quantum technologies, and nanoscale metrology, to name but a few. For health care, the Physics Laboratory conducts research on standards to enable hospitals to use nuclear medicine more effectively. We develop ways to image single biomolecules and to use terahertz radiation for measuring biomolecular processes.
The Physics Laboratory is at the forefront of the nascent field of quantum information processing-computing and communications - challenging preconceived notions of computational complexity and communications security. Similarly, the Physics Laboratory has been a leading center for metrology at the nanoscale, even before "nanotechnology" gained prominence. We pioneered electron-spin microscopy, which images magnetic materials, and our unique EUV optics facility supports the electronic industry in its drive to develop advanced lithographic systems for producing ever smaller chips.

As you browse this summary of the Physics Laboratory, we expect you will want to learn more. We invite you to visit our website, http://physics.nist.gov/, and we invite your inquiries and interest in measurement services and collaborations.

\section{Ketharine Cobbie}

Katharine Gebbie

Director, Physics Laboratory 


\section{GOAL}

TO SUPPORT EMERGINC

ELECTRONIC, OPTICAL,

AND NANOSCALE

TECHNOLOGIES

\section{ELECTRON AND OPTICAL PHYSICS DIVISION}

The strategy for meeting this goal is to improve measurement science and to

develop the measurements and standards needed by emerging science and

technology-intensive industries.

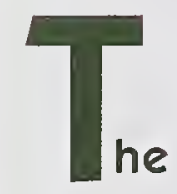

to develop techniques for

fabricating nanostructures

and measuring their

electronic and magnetic

properties.

\section{Nanoscale Electronics}

\section{and Magnetics}

\section{INTENDED OUTCOME AND \\ BACKGROUND}

The intended outcome of this work is the continuous improvement of methods for fabricating and characterizing nanometer-scale electronic and magnetic structures, as required to meet current and future needs of the semiconductor and data storage industries.
Our main tools for pursuing this program are scanning electron microscopy with polarization analysis (SEMPA) and the scanning tunneling microscope (STM). The Electron Physics Group in the Division has been a leading innovator in both of these methods, which are outgrowths of work begun at NIST in the 1970 s. SEMPA enables us to use conventional scanning electron microscopy (SEM) to image nanometerscale magnetic structure, through spin-polarization analysis of secondary electrons ejected from the sample. It has several unique capabilities that distinguish it from other magnetic imaging techniques: it is a highly sensitive, nonperturbative method, and thus is especially well suited for in situ studies of surface and nanostructure magnetization; it provides a direct measurement of the magnetization of a material region, rather than of a magnetic field; it has the high spatial resolution (about $10 \mathrm{~nm}$ ), long working distance, and large depth-of-field characteristic of SEM; and it facilitates simultaneous measurements of the magnetization and the topography. SEMPA studies have led to a number of breakthroughs in understanding the basic mechanisms of magnetism on the micro- and nanoscale, and have also addressed near-term measurement issues faced by the magnetic data storage industry.

Our STM program is focused on understanding the electronic and magnetic properties of nanostructures on surfaces. In recent years the STM program has been particularly concerned with the magnetic multilayer materials that have been investigated by SEMPA. The complementarities of SEMPA and STM measurements have elucidated many connections between conditions of layer growth and magnetic device performance.

The main, current direction of the STM program is the course of research made possible by the recently completed Nanoscale Physics Laboratory. This laboratory permits us to measure quantum electronic structures with atomic-scale imaging resolution and high electronenergy resolution. Samples grown in situ can be measured in an ultrahigh vacuum environment with magnetic fields of up to $10 \mathrm{~T}$ at temperatures down to $2.3 \mathrm{~K}$. Additionally, a program in Autonomous Atom Assembly is underway that will allow us to fabricate highly complex and perfect nanostructures on demand. 


\section{AC COMPLISHMENTS}

\section{Understanding Single Atom}

\section{Manipulation}

A low-temperature STM can move atoms about on clean, flat surfaces. However, our limited understanding of the interactions involved restricts our ability to make complex nanostructures.

We recently studied the atom dynamics that occur during atom manipulationimportant if we are to make real nanodevices on technological substrates.

We found that the atoms at the end of an iridium STM tip create a potential trap sufficient to capture a cobalt atom adsorbed on a clean copper crystal and to limit its lateral motion to less than $0.2 \mathrm{~nm}$. We also discovered that the vibrational level of the cobalt-copper bond could be varied dramatically by changing the electron current tunneling into the cobalt atom.

By adjusting the proximity of the STM tip to the cobalt atom, as well as the current flowing through it, it is possible to translate the cobalt atom across the copper crystal surface in a controlled and well-understood manner. It is even possible to force the cobalt atom into what is normally not a binding site on

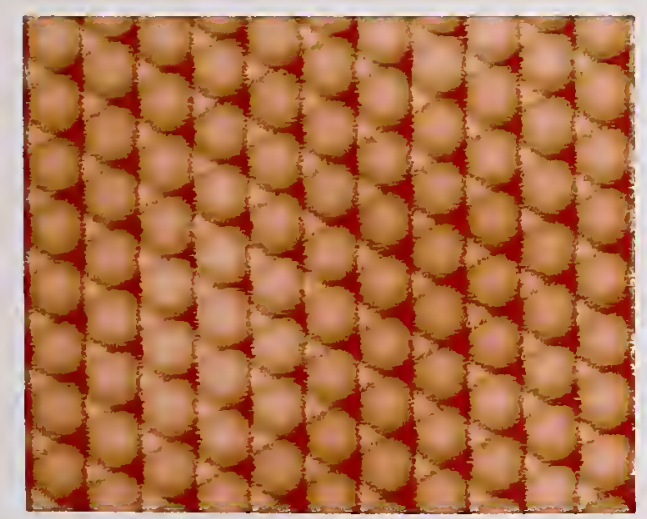

Figure 1. Binding site image for a cobalt atom on a copper (111) surface, showing three-fold symmetry. the surface. As the conditions necessary for forcing the atom into a nonbinding site are approached, the atom begins to jump back and forth between the nonbinding site and a neighboring binding site, giving rise to audio frequency noise in the tunneling current. This noise can be monitored in the laboratory and correlates with the locations of the nonbinding sites on the surface.

Using our newly acquired understanding of the atom manipulation process, we were able to: obtain a direct observation of a characterizable random two-state fluctuator, demonstrate the ability to modify single atom surface dynamics, show how chemical binding sites can be modified to change unstable ones to stable and vice versa, provide the first explanation for the origin of excess tunneling noise, and present a new type of STM image that reveals the surface binding sites of the crystal. (See Fig. 1.)

This work is an example of a new class of measurements referred to as "Atom-Based Metrology," where a single atom probes its local environment while nanoscale techniques position it within that environment. Here, the probe is the cobalt atom and the STM tip moves it across the copper surface. The atom responds to the surface potentials through its lateral motion, which is reflected in the measured tunneling current. This allows measurement of the binding site map of the copper surface through observation of the dynamical response of a single scanned atom to its environment. We are planning additional experiments using atombased metrology.

CONTACT: DR. JOSEPH A. STROSCIO (301) 975-3716 joseph.stroscio@nist.gov

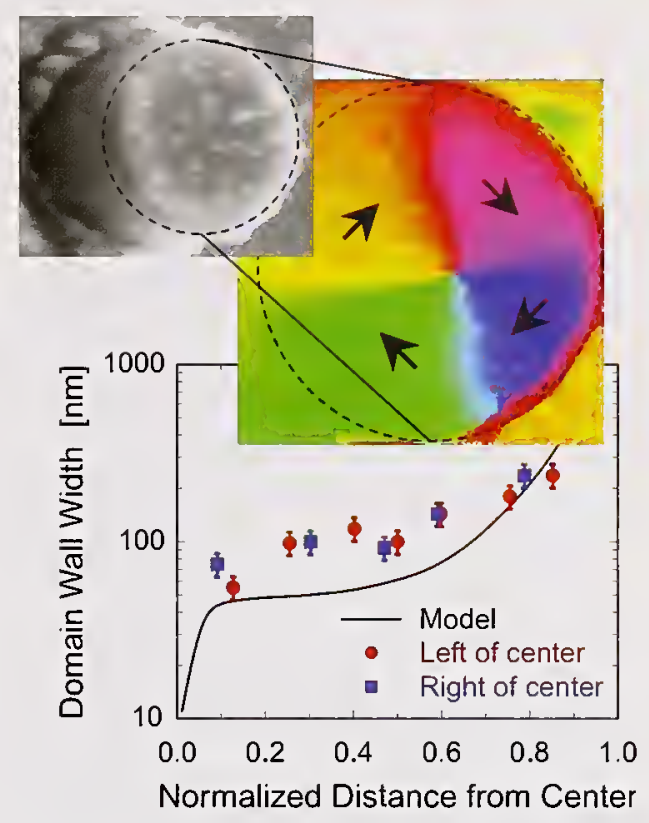

Figure 2. SEM image of a silicon nanopillar, along with a SEMPA image of the epitaxial, cobalt thin-film disk at the top of the pillar. The SEMPA measurement shows a geometrically constrained domain wall whose width varies with radial distance.

\section{Geometrically Constrained} Magnetic Nanostructures

Small, patterned, magnetic thin-film structures play a critical role in emerging magnetoelectronic technologies, which range from nonvolatile random access memories to magnetic field sensors. One potential advantage of miniaturizing these devices is that the size and geometry of the patterned structure may be used to shape the internal magnetic nanostructure and thus control the device operation. Imaging this internal nanostructure is a measurement challenge, since the structures consist of only a small amount of magnetic material and can easily be perturbed by conventional magnetic imaging methods such as Magnetic Force Microscopy (MFM). 
In collaboration with the University of Cambridge Thin-Film Magnetism Group we have used our SEMPA facility to noninvasively image the magnetic nanostructure of epitaxially grown thinfilm Co disks. Isolated, micrometersized Co disks were grown by depositing a several-nanometer-thick Co film onto a Si substrate with etched circular pillars. Unlike bulk Co, these epitaxially grown Co thin films have a cubic crystal structure that leads to a four-quadrant, closed-flux domain structure, characteristic of a film with cubic anisotropy.

The SEMPA images (e.g., Fig. 2) showed how the internal magnetic nanostructure of these disks depends critically on the size and shape of the element. Magnetic domain wall widths, for example, do not depend on sample geometry in bulk ferromagnets. But in these structures the domain walls are geometrically constrained by the significant shape-related magnetostatic energies, and the wall widths vary dramatically with radial distance from the magnetic vortex core.

\section{CONTACT: DR. JOHN UNGURIS}

(301) 975-3712

john.unguris@nist.gov

\section{Imasing Masnetic Sensors}

Recent advances in thin-film and multilayer magnetism offer the exciting possibility of creating a new generation of magnetic sensors that are small, inexpensive, and as sensitive as currently used, more cumbersome sensors such as SQUIDs. Development of this new generation of sensors requires understanding and control of the magnetic nano-structure, in order to enhance sensitivity and eliminate potential sources of magnetic noise.

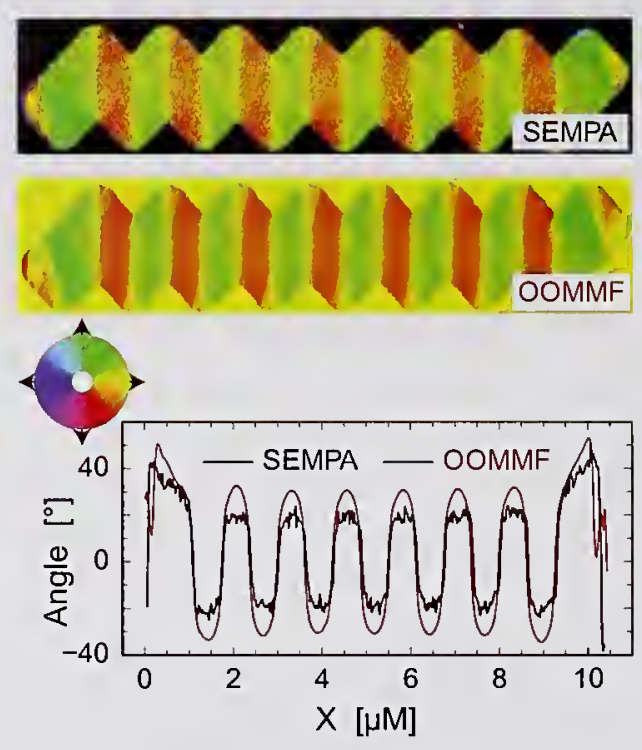

Figure 3. SEMPA image of a zigzag magnetic sensor element, compared with the OOMMF micromagnetics simulation. Line scans through the centers of the elements show the magnitude of the magnetic oscillations

We are working with NIST magnetics groups in EEEL, MSEL, and ITL to test and measure possible new materials and devices for use as sensors. One potentially useful device is a zigzag-shaped magnetic sensor developed by EEEL. The device's magnetic sensitivity is based on anisotropic magnetoresistance (AMR), and therefore requires the magnetization to be tilted with respect to the current passing through the device. In the zigzag sensor, the shape anisotropy forces the magnetization to tilt relative to the current.

We have used SEMPA to measure how well various zigzag geometries accomplish this angular biasing and to look for potential sources of magnetic noise. (See Fig. 3.) SEMPA imaging is especially useful since it not only images the magnetization directly, it provides a quantitative measurement of magnetization directions that can be compared to the results of micromagnetic calculations. The SEMPA images also reveal potential magnetic trouble spots such as trapped magnetic singularities, which can interfere with reproducible magnetic response, as well as defects that can produce magnetization-related noise.
Work is currently underway to allow imaging of electrically active magnetic sensors, which will allow a better understanding of noise sources and failure modes in real devices.

CONTACT: DR. JOHN UNGURIS

(301) 975-3712

john.unguris@nist.gov

\section{Current Induced Magnetic Switching}

In a magnetic multilayer, changing the relative orientation of the magnetizations of two layers changes the current flowing through the multilayer. This effect, called giant magnetoresistance, is used in magnetic sensors, magnetic random access memory (MRAM), and read heads in magnetic disk drives. An inverse effect, where the current changes the magnetic configuration, is being studied for possible applications. In this case, a current passing through a multilayer exerts a torque on the magnetizations of different layers causing the magnetizations to rotate.

Large enough currents passing through such multilayers can switch the magnetizations of the layers between parallel and antiparallel, or cause one to rapidly precess around the other layer. If the size of the current necessary to reverse magnetizations can be reduced sufficiently, spin-transfer torques could provide a way to switch bits in MRAM. In other devices, it should be possible to make current-controlled oscillators. 
We have developed quantitative models for these spin-transfer torques to help the development of devices based on this effect. (See Fig. 4.) In particular, we recently developed an analytic formula for the torque as a function of the device geometry and the magnetic configuration. Such a formula will allow rapid simulation of prototypes for device optimization.

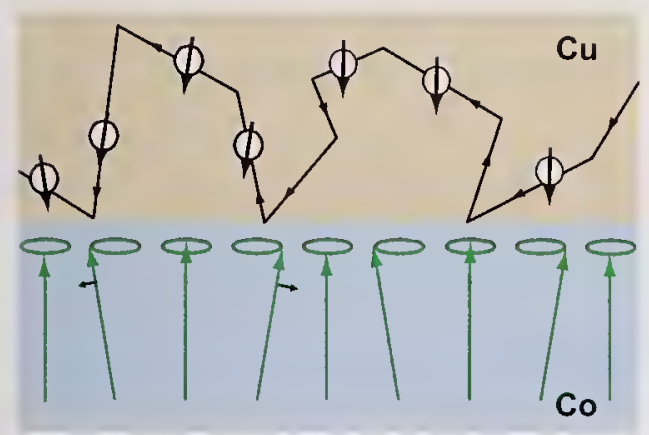

Figure 4. Electron diffusing near a ferromagnetic interface. The spin of the electron diffusing in the copper has its direction changed when it scatters from a cobalt interface with an in-plane spin wave.

We have also shown how spin-transfer torques can cause a magnetic instability even with a single ferromagnetic layer. This effect was first seen in point-contact experiments and later in more device-like, lithographically fabricated nanopillars. We explained how in-plane inhomogeneities in the magnetization lead to the observed instabilities. We are presently studying the effect of these inhomogeneities on more typical device geometries.

CONTACT: DR. MARK D. STILES

(301) 975-3745

mark.stiles@nist.gov

\section{End States in One-}

\section{Dimensional Chains}

The abilities to fabricate structures on the nanoscale and to measure their electronic properties present the opportunity to discover new phenomena. We have observed a new kind of electronic state at the ends of a one-dimensional nanostructure. This "end state" is a direct consequence of the lower dimensionality of the structure. Such end states can be thought of as zero-dimensional analogs to two-dimensional states that occur at the surface of a crystal.

\section{In fabricating these structures, we} exploit the self-assembly of atom chains that occurs when we deposit gold on stepped silicon surfaces at elevated temperatures. Rows of atom chains of varying length can be seen in the STM images of Fig. 5, which are of the same area but measured at opposite polarity. The chains in the upper panel, measured at a sample potential of $+0.5 \mathrm{~V}$, appear shorter than the chains in the lower panel, measured at a sample potential of $-1.0 \mathrm{~V}$, as emphasized by

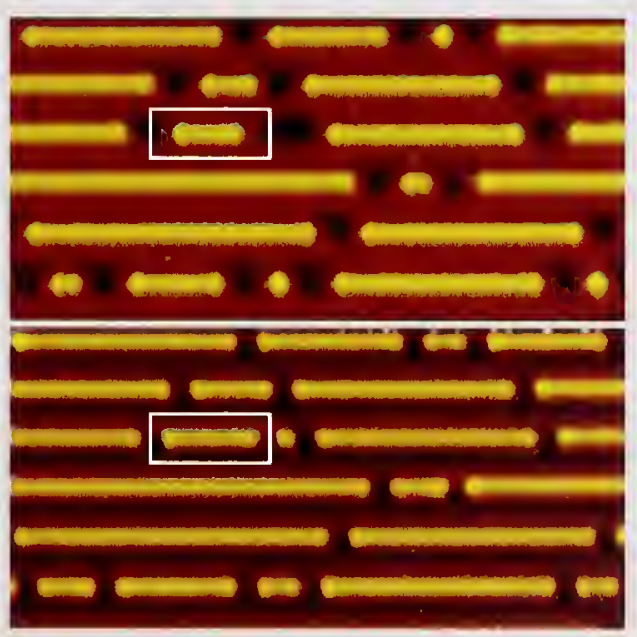

Figure 5. STM images of one-dimensional atom chains on Silicon, showing empty states (upper panel) and filled states (lower panel). End states cause the chains to appear shorter in the upper panel. the white box around a seven-atom chain. Such a polarity contrast in STM suggests an underlying difference in the density of states for the empty and filled states near the ends of the chains, indicating the presence of end states.

To characterize this new kind of electronic state, we made spatially resolved scanning tunneling spectroscopy measurements along finite chains to map the density of states. These measurements reveal quantized states that form in isolated chain segments. Furthermore, a transfer of spectral weight from the filled to the empty states over the atoms at the ends of the chains is directly attributable to the formation of end states.

These end states cannot be described by a simple particle-in-a-box model for states along the chains. A comparison to a tight-binding model demonstrates how the formation of end electronic states transforms the density of states and the quantized levels within the chains. The end states effectively lower the energy levels of the filled states within the chains, suggesting a possible driving force for their formation. As a further confirmation of the tight-binding model and the end electronic effects, we calculated STM topography profiles at positive and negative biases and reproduced the experimentally observed contrast at the end atoms in Fig. 5.

CONTACT: DR. DANIEL T. PIERCE

(301) 975-3711

daniel.pierce@nist.gov 
he second strategic

focus is the development of metrology for extreme ultraviolet (EUV) optics, the maintenance of national primary standards for radiometry in the EUV and adjoining spectral regions, and the operation of national user facilities for EUV science and applications.

\section{Extreme Ultraviolet Radiation Metrology}

\section{INTENDED OUTCOME AND BACKGROUND}

The intended outcomes of this program are: maintenance and continuous improvement of the national primary measurement standards for extreme ultraviolet radiation (EUV: wavelengths between $4 \mathrm{~nm}$ and $250 \mathrm{~nm}$, i.e., from soft $\mathrm{x}$ rays to vacuum ultraviolet), development of techniques for fabricating and characterizing EUV optical systems, and the development of a synchrotronbased, national primary standard for source-based optical radiometry.

The Division has longstanding responsibility for the national primary radiometric standards in the EUV region of the spectrum. EUV radiation is an important tool for determining the electronic structure of materials, diagnosing plasmas, measuring dynamics of the upper atmosphere, and probing the structure and dynamics of astrophysical objects.

One of the top candidates for next-generation semiconductor manufacturing technology is an EUV micropatterning tool, since operation at this short a wavelength (13 nm vs. $193 \mathrm{~nm}$ for present, production ultraviolet lithography) enables diffraction-limited imaging of features with smaller critical dimensions. We are working actively with the semiconductor industry to develop new metrology and testing capabilities as needs arise in their effort to commercialize this next-generation lithography.

The Division's key tool for EUV metrology is the NIST Synchrotron Ultraviolet Radiation Facility (SURF III). SURF III, the successor to the world's first dedicated source of synchrotron radiation, is a low-energy ( $<400 \mathrm{MeV})$, high beam-current (up to $1 \mathrm{~A}$ ), perfectly circular electron storage ring. Its operational characteristics are ideal for EUV metrology. It does not produce the hard $\mathrm{x}$-ray radiation of higher energy sources, and it can be operated over a wide range of beam energies to match the spectral response of systems of interest. As a calculable source of radiation from the far infrared through EUV spectral regions, SURF is also used as a primary standard for source-based radiometry throughout the optical spectrum.

\section{ACCOMPLISHMENTS}

\section{Characterization of Damaged EUV Condenser Optics}

EUV lithography is a candidate process for producing the next generation of semiconductor devices, with feature sizes that are $50 \mathrm{~nm}$ or less. One of the out-

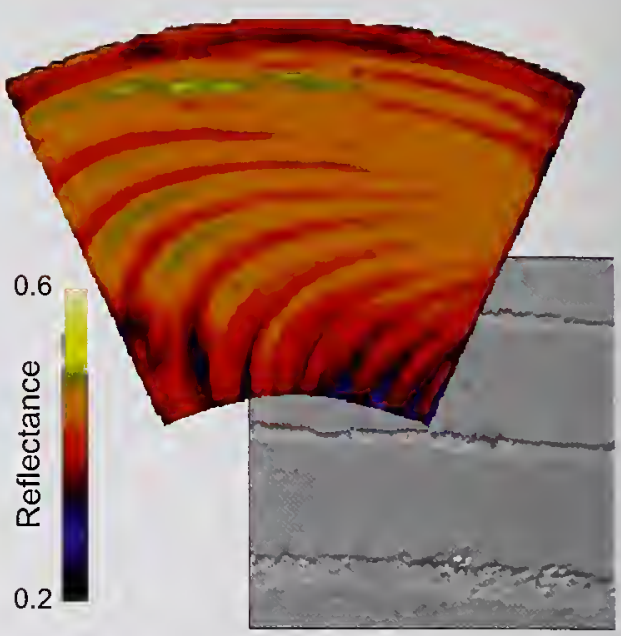

Figure 6. Measured EUV reflectivity over surface of a condenser optic, and an SEM image of a small region showing the striations associated with the erosion of the multilayers.

standing issues that must be solved in order for EUV lithography to proceed to commercialization is the durability of condenser optics, which collect and direct the radiation from its source. EUV radiation is produced by hightemperature plasmas generated by electrical discharges or high-intensity lasers. These plasmas emit not only EUV radiation, but also broadband radiation, electrons, and ions. This hostile environment can damage the condenser optics and reduce its lifetime, which must be months long for EUV lithography to be commercially viable.

In collaboration with SEMATECH we have analyzed one such condenser element, which had operated for three months in the Engineering Test Stand at Sandia National Laboratories. This condenser had a Mo/Si multilayer reflective coating, and it was used to collect the radiation from a laser-produced $\mathrm{Xe}$ plasma. After exposure to the plasma, the optic was covered by a visible array of erosion-associated striations. The origin of these stripes was a mystery.

As shown in Fig. 6, the reflectivity of the optic was measured at the NIST/ DARPA EUV facility, while the surface composition and structure were mea- 
sured using the Electron Physics Group's SEM, AUGER, and AFM facilities.

This combination of tools allowed an understanding of the optic's EUV reflectivity in terms of the nanoscale compositional and structural properties of the surface.

The measurements showed that the lowreflectance stripes were due to periodic stripes of increased roughness, and that each stripe corresponded to one additional $\mathrm{Mo} / \mathrm{Si}$ bilayer of the multilayer coating being removed.

\section{CONTACT: DR. SHANNON HILL.}

(301) 975-4283

shannon.hill@nist.gov

\section{Long-Term Endurance Testing of EUV Projection Optics}

The lithography tool that exposes the wafers in the patterning process is called a stepper. It consists of a radiation source and its condenser optics to illuminate the patterning mask, projection optics to image the mask onto a resistcoated wafer, and a mechanical device that aligns the wafer to the mask. Although the system is operated under vacuum, there is still a significant amount of water and hydrocarbons present. These materials interact with the EUV and cause the performance of the optics in the system to degrade. The effect is significant, and it is a critical factor in determining the operational lifetime of a stepper.

NIST recently commissioned a beamline on SURF III dedicated to longterm exposure studies of EUV multilayer projection optics. It contains a spherical, $75 \mathrm{~mm}$ diameter, $\mathrm{Mo} / \mathrm{Si}$ multilayer

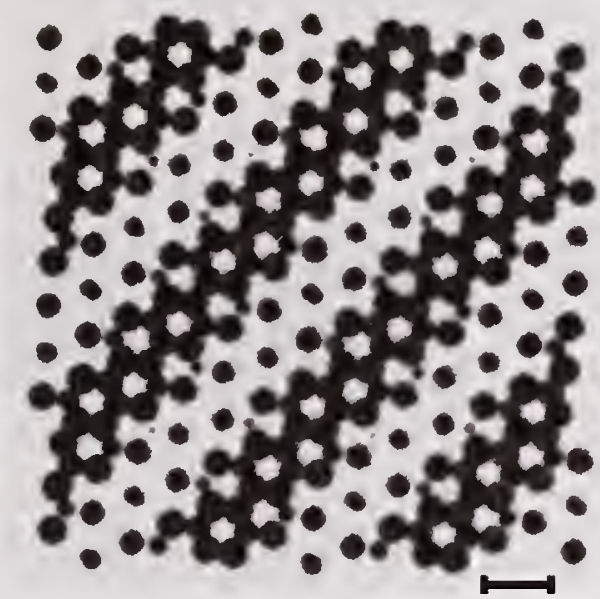

Figure 7. Simulated reconstruction of a polymer sample, as imaged by transmission electron microscopy. Length of bar in lower right is 1 micrometer.

focusing mirror. This images the EUV beam onto a sample optic with a spot size of approximately $0.6 \mathrm{~mm} \times 0.8 \mathrm{~mm}$ (FWHM). The sample chamber is separated from the mirror chamber with a removable, $0.3 \mu \mathrm{m}$ thick beryllium foil. It filters out long-wavelength radiation and allows a differential vacuum between the sample chamber and SURF. This permits exposure of the sample optics to EUV radiation in the presence of larger partial pressures of water and other species, to simulate the environments present in EUV steppers.

CONTACT: DR. CHARLES S. TARRIO

(301) 975-3737

charles.tarrio@nist.gov

\section{Nanoscale Chemical Imaging}

We are developing state-of-the-art measurement techniques and algorithms to acquire three-dimensional, chemically resolved images of nanoscale samples, using transmission electron microscopy. We seek an enhanced, fundamental understanding of the probe-sample interactions relevant to electron microscopy, including incoherent and coherent scattering, electron energy loss spectroscopy, and x-ray generation and propagation. In this joint project, we use instrumentation to acquire data in the Chemical Science and Technology Laboratory and investigate reconstruction algorithms to form the images with the Information Technology Laboratory.

Earlier work led to reconstructions of integrated circuit interconnects using an $\mathrm{x}$-ray microscope, as well as to the detailed observation of $\mathrm{M} \mathrm{x}$-ray absorption edges in tantalum and tungsten. More recently, the possibility of performing reconstructions on polymer samples up to 8 micrometers in size using a scanning transmission electron microscope has been demonstrated in simulation. The total size is noteworthy because nearly all tomographic reconstructions use smaller samples to avoid the multiple-scattering regime. Here it is shown that multiple scattering can be accounted for mathematically, and reconstructions may be performed, even in the presence of a few scattering events. A simulated reconstruction appears in Fig. 7.

CONTACT: DR. ZACHARY H. LEVINE (301) 975-5453

zachary.levine@nist.gov

\section{Absolute Radiometry at SURF I\|}

The SURF III facility recently became the source of the most accurate ultraviolet lamp calibrations in the world.

The SURF upgrade in the mid-1990s was motivated by the need to improve our source calibration capabilities in the ultraviolet spectral region. To accomplish this, the magnet system had to be improved, which in SURF's case meant replacement of the coils, the steel used in the back legs, the power supply, and the control system. 
Recently these immense improvements were put to the test during a Consultative Committee for Photometry and Radiometry (CCPR) key comparison between several national measurement institutes, piloted by the German Physikalisch-Technische Bundesanstalt (PTB). This comparison focused on the calibration of specially prepared deuterium lamps in the spectral range from $200 \mathrm{~nm}$ to $350 \mathrm{~nm}$.

NIST was the sole laboratory to employ synchrotron radiation in the calibration process, leading to the smallest uncertainties among all participating laboratories. The uncertainty achieved at SURF was $0.5 \%$ (coverage factor $k=1$ ) for the entire spectral range.

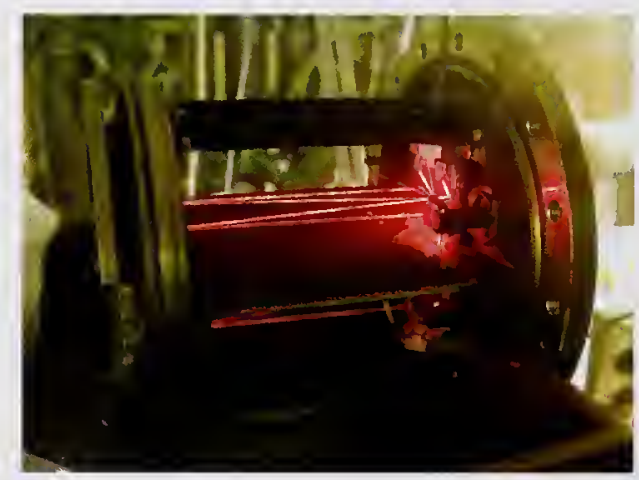

Figure 8. Monochromator during alignment.

For this comparison, a detection system, consisting of a monochromator, a diffusing device, and a photodetector, was calibrated against SURF, utilizing the calculability of synchrotron radiation in conjunction with our accurate monitoring of its operational parameters.

Figure 8 shows a monochromator during alignment. Once the system was calibrated, it could be used to determine the spectral irradiance of a lamp.

CONTACT: DR. UWE ARP

(301) 975-3233

uwe.arp@nist.gov he third strategic focus is metrolosy for coherent matter-wave and quantum information processing devices.

\section{Coherent Matter-Wave and Quantum Information \\ Processing Metrology}

INTENDED OUTCOME AND
BACKGROUND

This program provides measurements and data to enable the development of ultracold atom technology, in particular the use of coherent matter waves in sensors, atom interferometers, and quantum information processing devices.

The Division maintains two efforts in this area, one theoretical and one experimental. The theoretical program is focused on quantitative modeling of degenerate quantum gases, with particular attention to the dynamics of BoseEinstein condensates subject to external forces, e.g., manipulation of condensates confined in an optical lattice. This program is an outgrowth of extensive collaborations with experimental groups at NIST, JILA, and elsewhere, begun in the mid-1990s.

The experimental program develops deterministic atom-delivery systems, i.e., devices that can deliver precisely one atom to a predetermined location, on demand. In addition, the Division is developing a testbed for quantum communication systems, together with the Atomic Physics Division and the Information Technology Laboratory.

\section{ACCOMPLISHMENTS}

\section{Characterization of \\ "Atom-on-Demand" Source}

Recent developments in our laboratory have led to a new source of atoms that is almost completely deterministic: single atoms can be made available at a high rate with near unity certainty. Such a source has applications not only in quantum information processing, where single atoms can be used as qubits, but also in nanotechnology, when doping of nanostructures reaches the level where each nanostructure contains only a countable number of dopants.

In order to proceed to the next step in our research program, which will be to extract atoms from our source and demonstrate controlled deposition, we have conducted a number of studies to characterize our source. These have involved a detailed analysis of all the experimental parameters that determine the performance of the source, and also real-time photography of single atoms.

The detailed analysis has allowed us to implement Monte Carlo simulations that show extremely good agreement with experimental observations of such parameters as the single-atom probability and the single-atom extraction success rate. The photography, using a high-sensitivity CCD camera (Fig. 9), has given us the ability to investigate the spatial properties of our source, which will prove crucial in extracting, transporting, and depositing the single atoms with high spatial resolution.

CONTACT: DR. JABEZ J. MCCLELLAND (301) $975-3721$ jabez.mcclelland@nist.gov 
Random numbers of atoms...

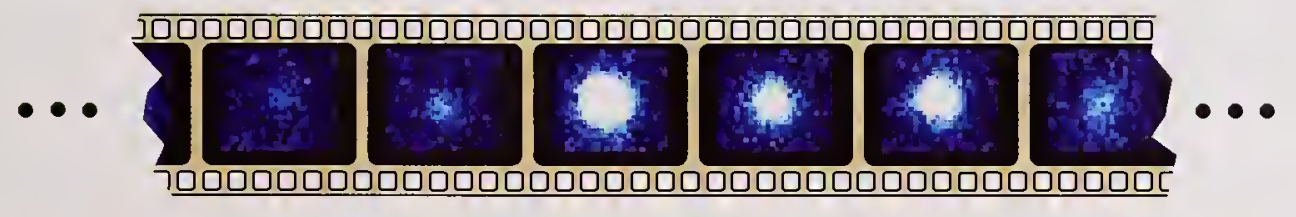

Single atom on demand...

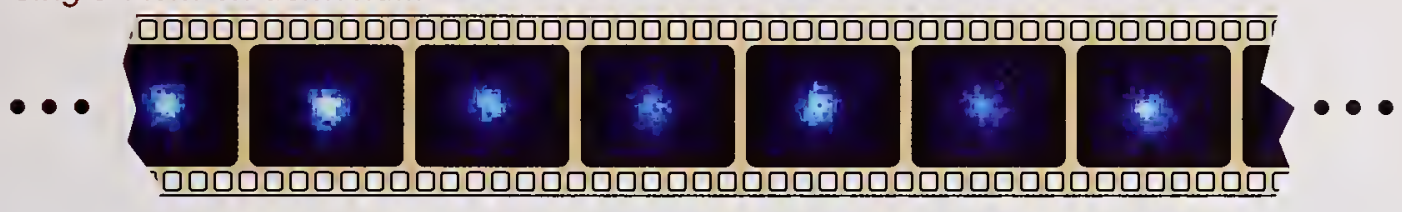

Figure 9. Real-time images of single atoms in an atom-on-demand source. Without feedback, the number of atoms is random, fluctuating between one, two, and three. With feedback, there is always just a single atom in the source.

\section{Resonant Atom-Molecule}

\section{Mixtures in Quantum-}

\section{Degenerate Gases}

Cooling fermionic, atomic gases to quantum degeneracy, in conjunction with the ability to directly control the interatomic interactions through magnetic-field-tunable Feshbach resonances, has opened the door to a detailed study of the crossover between BardeenCooper-Schrieffer superfluidity and Bose-Einstein condensation of diatomic molecules in an atomic Fermi gas.

A Feshbach resonance can be used to couple atom pairs into a bound molecular state, thereby creating bosonic dimers in the gas. Under suitable conditions, these dimers can undergo BoseEinstein condensation. The BCS-BEC crossover is a cornerstone problem of condensed matter physics, due in large part to its importance in the understanding of high-temperature superconductivity.

We have studied the equilibrium phase diagram of atom-molecule mixtures in a simple model under experimentally relevant conditions. A finite fraction of molecules persists for energies above dissociation threshold. At high temperatures this is ascribable to a detailed balance between atomic association and molecular dissociation in the gas, while in the quantum-degenerate regime Pauli blocking stabilizes molecular states embedded in the continuum.

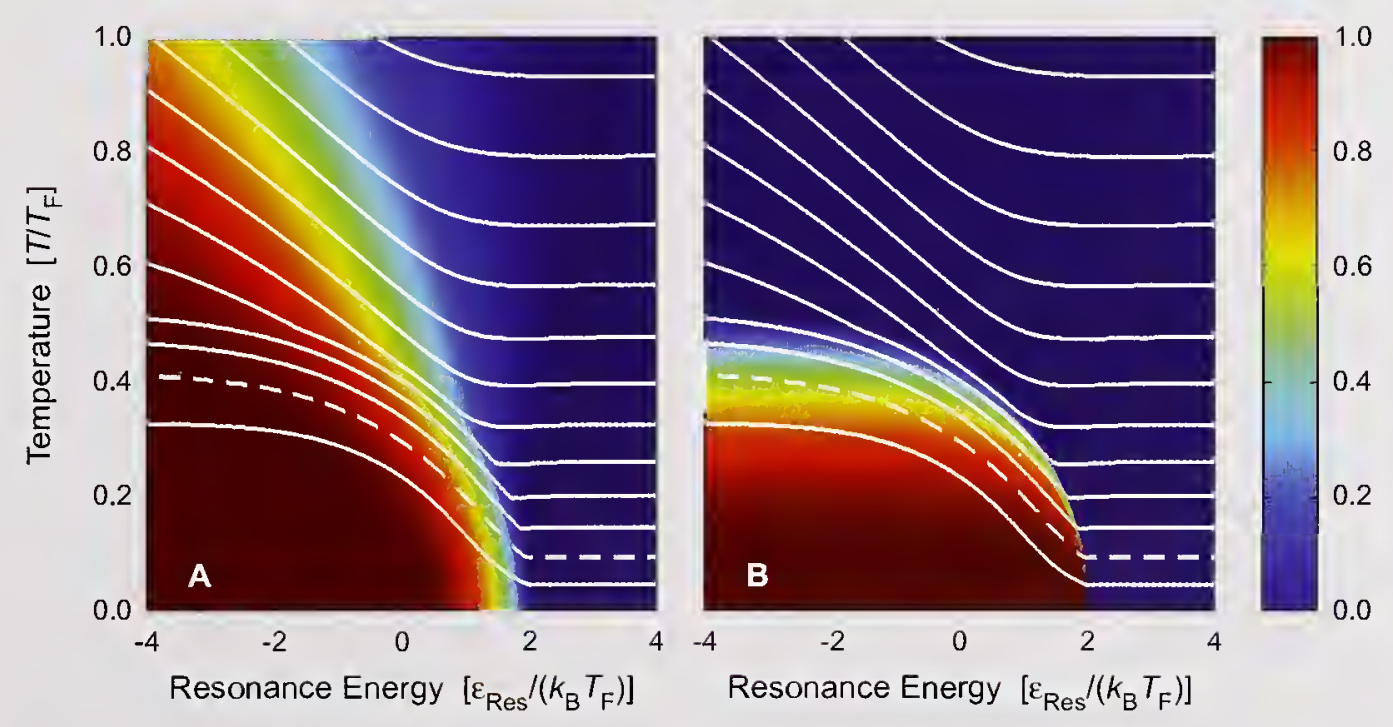

Figure 10. Phase diagrams of atom-molecule mixture showing the molecule fraction $(A)$ and condensate fraction (B) as a function of temperature and resonance energy, $\varepsilon_{\text {Res }}$ in units of the Fermi temperature and Fermi energy, respectively. Lines indicate contours of constant entropy, which are the trajectories followed by the system under adiabatic variation of the threshold energy. 


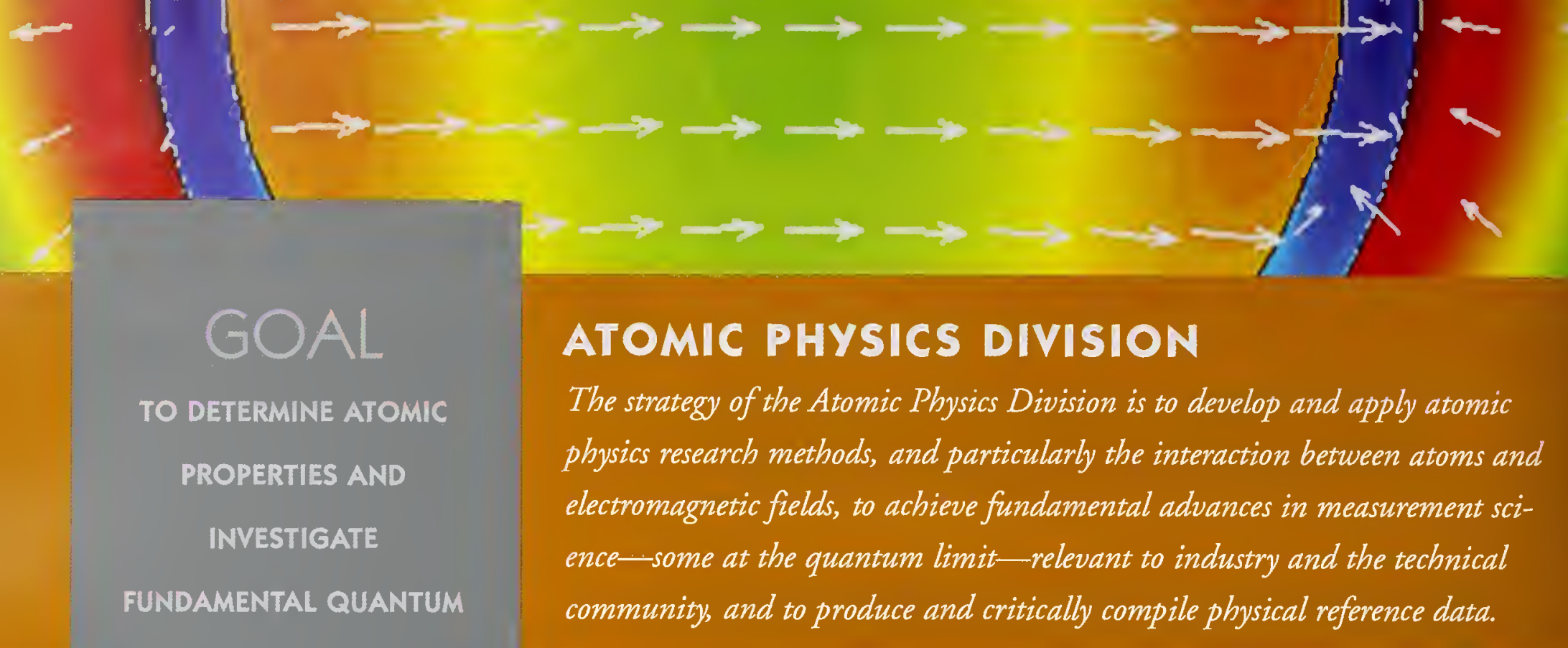

INTERACTIONS

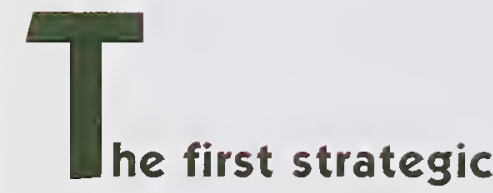

element is to advance the physics of electromagneticmatter interactions, to explore new applications for laser cooled and trapped atoms, to study exotic states of matter, and to study and control many-body quantum systems.

\section{Light-Matter Interactions and Atom Optics}

\section{INTENDED OUTCOME AND BACKGROUND}

This strategic element focuses on the physics of laser cooling and electromagnetic trapping of neutral particles, the manipulation of Bose-Einstein condensates (BECs), and the use of optical dipole forces as a new tool for analyzing microscopic objects in biochemistry. It includes both fundamental and applied studies, such as developing measurement techniques for biomolecular systems and developing a quantum information processor. A strong theoretical-experimental collaboration is aimed at interpreting experimental results and providing guidance for new experiments.

The development of laser cooling and trapping techniques allows exquisite control over the motion of atoms. Such control has been exploited to build more precise atomic clocks, gravity meters, and gravity gradiometers. These techniques also enable the study and manipulation of atoms and molecules under conditions in which their quantum or wave behavior dominates. This research has revolutionized the field of matterwave optics.

Our research includes theoretical and experimental projects that contribute to the understanding and exploitation of: Bose-Einstein condensation of neutral atoms; matter-wave optics; optical and magnetic control of trapped, ultracold atom collisions; advanced laser cooling and collision studies for atomic clocks; the quantum behavior of atoms in optical lattices, including in low dimension- ality; the superfluid to Mott-insulator quantum phase transition; quantum information processing; quantum computing architectures; and optical characterization and manipulation of single molecules, biomolecules, and biomembranes.

\section{ACCOMPLISHMENTS}

\section{Control of Cold Quantum Gases}

Many recent atomic physics experiments have used magnetic field control of scattering resonances to modify the properties and dynamics of ultracold atomic quantum gases such as Bose-Einstein condensates or mixtures of fermions. These resonances occur when the energy of a bound state of two atoms is tuned to the same energy as that of two separated cold atoms. Such resonances can be used to make cold molecules and molecular Bose-Einstein condensates, to strongly modify the nature of superfluid atom pairing, and to modify the properties of atoms trapped in optical lattices. Such phenomena are relevant to fundamental physics, condensed matter (solid state) physics, atomic clocks, and quantum information. 
We have applied quantum mechanical models to quantitatively characterize such resonances and to develop simple physical models for understanding them. These have been applied to give good agreement with data and predictive power for bosonic species ${ }^{85} \mathrm{Rb}$ (see Fig. 1) and ${ }^{133} \mathrm{Cs}$, and for fermionic species ${ }^{6} \mathrm{Li}$ and ${ }^{40} \mathrm{~K}$. We have also initiated studies on optically induced scattering resonances for laser control of quantum gases of alkaline earth species such as $\mathrm{Ca}$ or $\mathrm{Sr}$, which are of great interest for next-generation atomic clocks.

CONTACT: DR. PAUL S. JULIENNE (301) 975-2596

paul.julienne@nist.gov

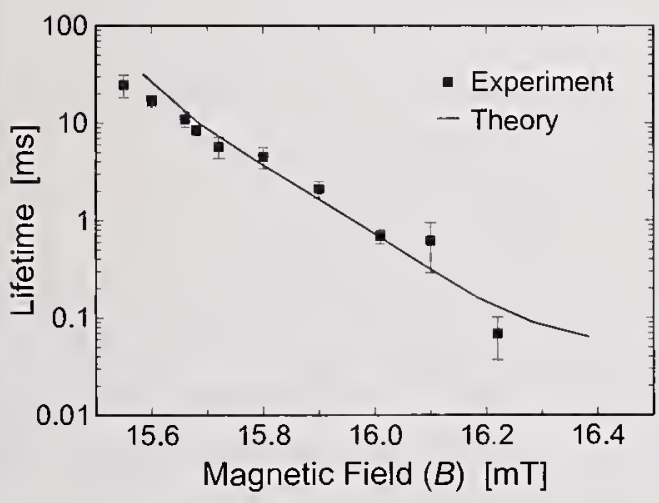

Figure 1. Lifetime of the tunable, molecular bound state of the ${ }^{85} \mathrm{Rb}_{2}$ dimer as a magnetic field $B$ is varied near a scattering resonance at $15.2 \mathrm{mT}$. The rapid increase in lifetime is due to the dramatic increase in the size of the weakly bound atom-pair, to hundreds of nanometers, as the field gets closer to the resonance value.

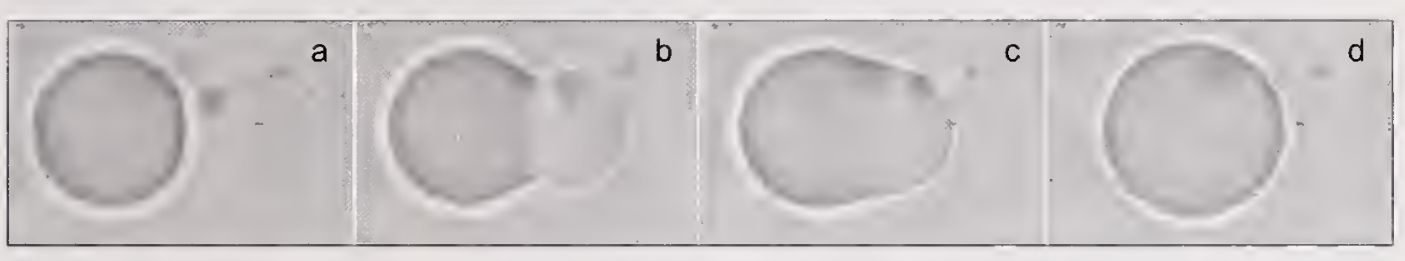

Figure 2. Time sequence of video microscopy images showing two liposomes, brought into contact with optical tweezers and induced to fuse by an optical scalpel, a pulsed ultraviolet laser focused on the contact point, which initiates the fusion (a). Images (b). (c), and (d) were taken 132 ms, 264 ms, and $528 \mathrm{~ms}$ later, respectively.

\section{Spectroscopy of $\mathrm{Na} 2$ Molecules in the Lowest \\ Triplet State}

We have used the technique of twocolor photoassociation spectroscopy (two colliding atoms absorbing photons to create a diatomic molecule) to improve the spectroscopy of the lowest triplet state of the $\mathrm{Na}_{2}$ molecule. The spectra obtained represent a factor of 1000 improvement in resolution over the previously obtained spectra of this state, and include a number of previously unobserved vibrational levels, as well as unresolved hyperfine and fine structure. This knowledge has altered our view of the angular momentum coupling scheme that must be used to represent this state of the molecule.

Knowledge of the interaction potentials between two sodium atoms, at the level of detail that can be obtained by photoassociation spectroscopy, is required to predict the behavior of this prototype system of two colliding, effectively single-electron atoms. Improved theory in this area is necessary for understanding many experiments in atomic and molecular physics, and is especially pertinent for current studies of atomic and molecular Bose-Einstein condensates.

CONTACT: DR. PAUL D. LETT

(301) 975-6559

paul.lett@nist.gov

\section{Optical Manipulation of Nanocontainers for Biotechnology}

In collaboration with researchers in CSTL and CARB, we are developing optical techniques for manipulating nanocontainers (containers with femtoliter volumes of fluids), to perform ultrasmall volume chemistry and to isolate and sort single molecules.

We are currently investigating three systems, liposomes, polymersomes, and hydrosomes, for use as nanocontainers. Liposomes and polymersomes are closed structures composed of a lipid and polymer membrane, respectively, typically about 10 micrometers in diameter. The membrane acts as a barrier to separate an aqueous interior environment from an aqueous exterior environment. Hydrosomes are micrometer-sized, surfactant-stabilized water droplets that reside in a fluorocarbon environment.

The techniques we use include optical tweezers, for trapping and remotely moving the nanocontainers, and an "optical scalpel," for opening membranes in order to induce fusion of liposomes and polymersomes. (See Fig. 2.) In all three systems, we are able to bring together two similar nanocontainers using optical trapping and to subsequently fuse them together, allowing their contents to mix. 
We have demonstrated the use of liposomes and hydrosomes in performing controlled, elementary chemical reactions. We are currently investigating the application of these nanocontainers for single-molecule studies and singlemolecule sorting.

CONTACT: DR. KRISTIAN HELMERSON (301) $975-4266$

kristian.helmerson@nist.gov

\section{Neutral-Atom Quantum Information}

Single atoms can act as qubits for quantum information (QI) using, for example, the internal hyperfine and Zeeman states as the qubit states. Our approach to neutral-atom QI is to hold and manipulate the atoms using laser fields for trapping and transporting. An optical lattice provides a natural register for atomic qubits. A BEC loaded adiabatically into an optical lattice provides, potentially, millions of qubits initialized in their ground states, since the BEC, or at least the condensed fraction, is initially in the ground state.

If a noninteracting BEC is slowly loaded into an optical lattice, the typical result is that the coherent wavefunction of each atom is spread across all the available lattice sites, so that there is a statistical (Poissonian) fluctuation in the number of atoms in any given site. If, however, there are repulsive interactions, at some lattice depth the competition between tunneling and the on-site repulsive interaction favors the ground state being one (or some fixed number of) atom(s) per lattice site. This is a phase transition known as the Mott insulator transition because the system goes from being superfluid (at large tunneling rate) to insulating (at small tunneling).

We observe the phase transition for $\mathrm{Rb}$ atoms in a three-dimensional optical lattice by looking at the diffraction pattern formed by the atoms upon release from the lattice. As shown in Fig. 3, wellresolved diffraction indicates phase coherence across the lattice, and therefore an uncertain atom number in each site. The disappearance of the diffraction pattern is characteristic of a fixed number per site, and therefore an uncertain phase difference from site to site, indicative of being in the insulator state.

CONTACT: DR. WILLIAM D. PHILLIPS

(301) 975-6554

william.phillips@nist.gov

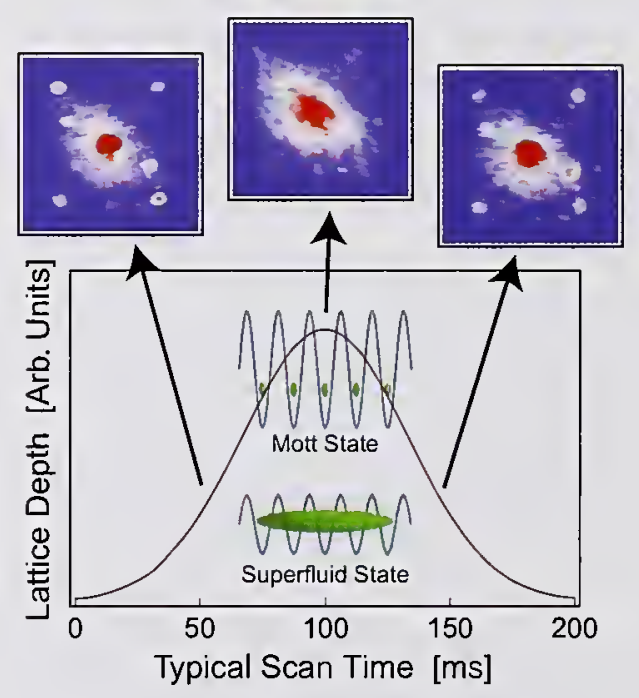

Figure 3. As the 3-D lattice depth is increased, the tunneling between lattice sites decreases. At a lattice depth of around 10 recoil energies, the system undergoes the Mott transition, and the diffraction pattern disappears. The transition is reversible, and the diffraction reappears when the lattice depth is reduced. he second strategic element is to advance measurement science at the atomic and nanometer scale, focusing on precision optical metrology, quantum devices, nanoscale plasmas, and nanooptical systems.

\section{Nanoscale and Quantum Metrology}

\section{INTENDED OUTCOME AND BACKGROUND}

This strategic element focuses on developing and exploiting precision metrology at the interface between atomic and nanoscale systems. Systems under study include quantum dots and wires, ultracold atomic quantum gases, metallic nanoparticles, and those with nanoscale features induced on surfaces by highly charged ions. Such systems arise in advanced $193 \mathrm{~nm}$ and $157 \mathrm{~nm}$ lithography, plasma etching of semiconductor wafers, nanolasers, detectors, biomarkers and sensors, nanomaterials, quantum devices and quantum information, and atomic clocks.

Our research combines theory and experiment. Theory is used to extend the fundamental understanding of systems at the atomic/nanoscale interface as necessary to interpret experiment, to explore new applications in nanoscale and quantum technologies, and to motivate new and enhanced precision metrology. We are developing the theoretical understanding needed to 
create nanooptics structures that will be needed in emerging quantum and nanoscale technologies.

Experiment is used to develop new precision measurement tools for this regime, to collect precise data essential for the applications mentioned, and to further the understanding of these sysrems. We have developed precision metrology to make accurate displacement measurements to subatomic dimensions by use of frequency combs locked to a cesium atomic clock. We have made the precise measurements of the refractive index of water needed by the semiconductor industry to develop immersion lithography for sub- $100 \mathrm{~nm}$ optical lithography. And we are now expanding our expertise by beginning to probe the nanooptics and nanomechanical properties of nanoscale and quantum-coherent systems.

\section{AC COMPLISHMENTS}

\section{Designing the Nanoworld:}

Nanostructures, Nanodevices,

\section{and Nanooptics}

Developing and exploiting precision metrology for quantum and nanotechnology requires nanoscale modeling of ultrasmall structures, devices, their dynamical operation, and their response to probes.

Atomic-scale simulations of the electronic and optical properties of complex nanosystems at the nano/molecular interface are being carried out. These systems include nanocrystals, self-assembled dots, nanodot arrays and solids, molecular electronics, and bio/nanohybrids. These simulations provide benchmarks for precise experimental

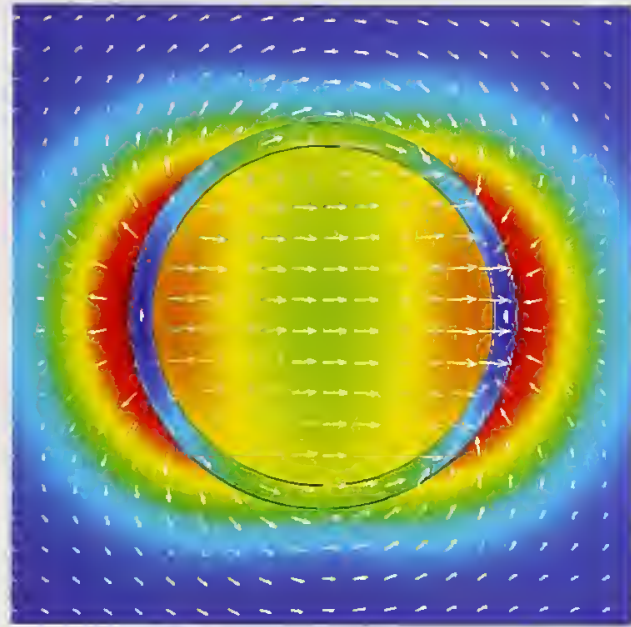

Figure 4. Field withın and around a $60 \mathrm{~nm}$ radius gold nanoring. Enhanced fields inside the nanoring would make the nanoring a use ful container for precision nanoexperiments.

tests of the atomic-scale sensitivity of nanosystems. The work is providing the foundation needed to build design tools for engineering nanolasers, detectors, biomarkers and sensors, quantum devices, and nanomaterials.

Nanoscale simulations of optical fields near nanosystems are also being carried out. Results are being used to design nanoprobes and nanocavities, as shown in Fig. 4, for use in precision nanooptics metrology. Results are being used to design and model the nanooptics highway, that is, a collection of nanoparticles used to generate, transport, and collect photons on the nanoscale, well below the diffraction limit that governs the classical transport of photons.

Nanooptics highways will be critical for the transport of excitations in quantum devices and in the metrology of these devices.

CONTACT: DR. GARNETT W. BRYANT

(301) 975-2595

garnett.bryant@nist.gov

\section{Subatomic Displacement}

\section{Measurements}

We are concluding our program to improve the accuracy of Michelson and Fabry-Perot interferometry for measuring displacements of macroscopic objects with subatomic precision. Our work in Fabry-Perot interferometry is the realization of the idea first put forth by Zoltan Bay at NBS in 1970, of using a microwave measurement of the mode spacing within a Fabry-Perot cavity to measure displacements free of the systematic error induced by diffraction.

In our most recent implementation of Bay's idea, as shown in Fig. 5, we probe two adjacent modes of a variable-length Fabry-Perot cavity with red light from a $\mathrm{HeNe}$ laser at $633 \mathrm{~nm}$, and another mode with orange light from a $\mathrm{HeNe}$ laser at $612 \mathrm{~nm}$. A precise measurement of the mode spacing using the light at $633 \mathrm{~nm}$ allows us to determine the exact number of modes in the $16 \mathrm{THz}$ interval between the red and orange lasers. We then beat light from the HeNe lasers against light from a visible frequency comb that is locked to a cesium atomic clock. The resulting measurements of the cavity length are not compromised by diffraction. They are related directly to the definition of the second, and by extension, the meter. 
We are now turning our focus to the metrology needs of microscopic systems themselves, in particular, microelectromechanical (MEMS), nanoelectromechanical (NEMS), and nanooptical systems. Precision optical and quantum optical metrology will be exploited to probe individual nanoscale and, possibly, quantum-coherent systems.

CONTACT: DR. JOHN R. LAWALL

(301) 975-3226

john.lawall@nist.gov

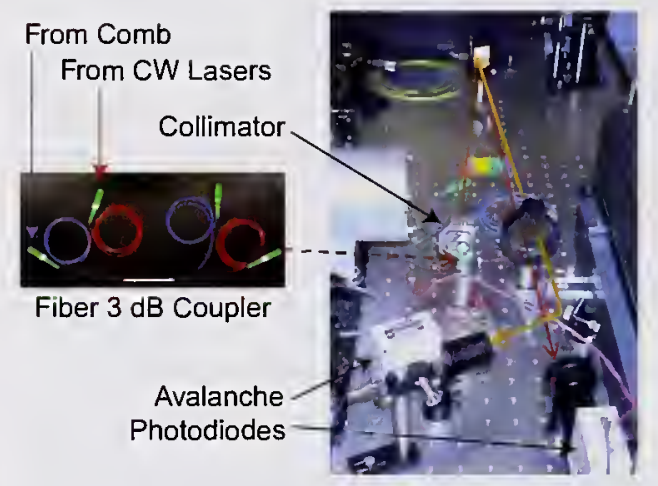

Figure 5. Setup to measure frequency difference of $16 \mathrm{THz}$ between lasers at $612 \mathrm{~nm}$ and $633 \mathrm{~nm}$. Light from the CW lasers is beat asainst light from a visible frequency comb in a fiber $3 \mathrm{~dB}$ coupler. After collimation, the red and orange components are separated with a grating, and the beats of the CW lasers with adjacent comb lines are measured on avalanche photodiodes.

\section{Optical Properties in Support of UV Lithography}

In late 2002, the semiconductor lithography industry began an effort to explore the feasibility of developing immersion lithography as a method to extend optical lithography into the sub$100 \mathrm{~nm}$ feature-size region. In this technology, an immersion fluid is inserted between the final lens of a $193 \mathrm{~nm}$ or $157 \mathrm{~nm}$ lithography projection system and the silicon wafer, thus dividing the diffraction-limited feature size by the refractive index of the fluid. However, at that time there existed no accurate measurements of the key optical properties of water, the principal candidate $193 \mathrm{~nm}$ immersion fluid.

To address this need, the Atomic Physics Division and the Optical Technology Division teamed up to develop a UV fluid refractive index measurement facility. By March of 2003, the project delivered the first accurate measurements of the refractive index of distilled water at $193 \mathrm{~nm}$ to five decimal places, sufficient for system design. The project also delivered other key optical properties of water, including the dependence of the index on wavelength, temperature, pressure, and gas and impurity content.

Immersion lithography systems using water at $193 \mathrm{~nm}$ are being designed and built around the world, relying on these measurements as key design parameters. The project has also characterized the optical properties of other fluids with significantly higher $193 \mathrm{~nm}$ indices than that of water, which may potentially enable further resolution increases. The semiconductor industry now has a parallel effort to explore immersion lithography at $157 \mathrm{~nm}$. The project has also delivered the first measurements of the key optical properties of the candidate immersion fluids in this vacuum ultraviolet region.

\section{CONTACT: DR. JOHN H. BURNETT} (301) 975-2679

john.burnett@nist.gov

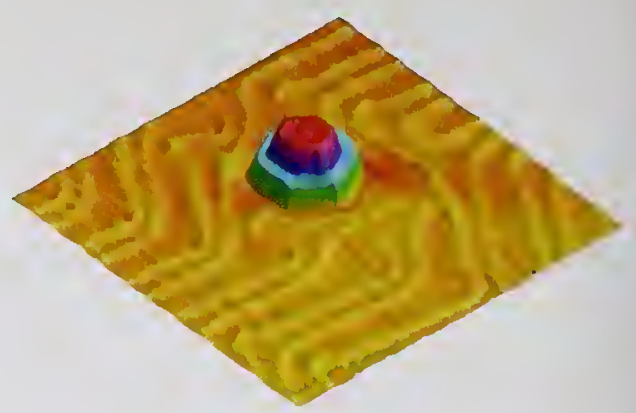

Figure 6. Scanning tunneling microscope image ( $32 \mathrm{~nm} \times 32 \mathrm{~nm}$ ) of a $9 \mathrm{~nm}$ mound, two atomic layers high, created by the impact of a single $\mathrm{Xe}^{44+}$ ion on the Au(111) surface. The mound has relaxed to a perfect hexagon, and the "herring-bone" reconstruction in the background has rearranged around the mound to minimize surface energy.

\section{Nanometrolosy of Highly- Charged-Ion Collisions with Neutral Matter}

Highly charged ions (HCIs) constitute a unique and exotic form of matter, typically out of human reach, but they can be created and studied within the NIST Electron Beam Ion Trap (EBIT) facility. When neutral matter is exposed to $\mathrm{HCIs}$, the intense electronic interaction produces extreme conditions that allow us to probe properties of both participants, including processes of $\mathrm{x}$-ray emission, charge exchange, and surface damage (see Fig. 6). By coupling HCIs with surface science techniques like scanning tunneling microscopy, we are able to observe electronic and morphological changes at atomic length scales and to probe the fundamental connections of atomic and solid state physics.

This unique combination of capabilities has been used to diagnose $\mathrm{HCI}$ damage to advanced $\mathrm{Mo} / \mathrm{Si}$ multilayer mirrors, used for extreme ultraviolet (EUV) lithography. Tunneling spectroscopy and EUV reflectivity measurements have revealed that the EUV-source ions cause approximately ten times more damage if they neutralize on the oxidized ruthenium capping layer of the optics than if they neutralize prior to collision.

These measurements highlight the broader need for providing critical components with protection from $\mathrm{HCIs}$ 
when operating in close proximity to plasmas. Experiments currently underway on gold surfaces are designed to determine whether high-electron-density materials exhibit less damage than insulating and oxidized materials, currently used for critical components.

CONTACT: DR. JOSHUA M. POMEROY (301) 975-5508

joshua.pomeroy@nist.gov

he third strategic element is to produce reference data on atomic structure, to critically compile reference data for scientific and technological applications, and to develop techniques to apply the data to further the understanding of important plasma devices.

\section{Critically Evaluated} Atomic Data

\section{INTENDED OUTCOME AND}

BACKGROUND

The objective of this strategic element is to critically compile fundamental constants and atomic spectroscopy data from the far infrared to the $\mathrm{x}$-ray spectral regions. We disseminate these reference data on the Physics Laboratory website, produce high-quality data for urgent scientific or technological needs, and resolve discrepancies in the body of the data. When reliable data do not exist for high-priority needs, specific measurements or calculations are undertaken to produce them.

The NIST databases for atomic spectra and fundamental constants are recognized throughout the world. The Atomic Spectra Database on our website now dispenses about 100,000 downloads (answers) per month, up from 80,000 only two years ago. The principal users are plasma physicists, crystallographers, astronomers, lighting engineers, and spectrochemists.

The newest version of the Atomic Spectra Database significantly reduces internal inconsistencies between atomic energy levels and atomic transition frequencies by merging older databases and resolving and removing inconsistencies. However, the databases remain far from complete, and the quality of the data available in the literature from which the databases are built is still uneven. The current versions of our databases are not sufficiently reliable for some fields of science and technology, and needs for such reference data are continuously growing. Our scientists focus their resources on the most urgent needs of the user communities.

\section{ACCOMPLISHMENTS}

\section{New Generation of Atomic Spectra Database}

Version 3.0 of the NIST Atomic Spectra Database has been released, which contains many new data and interface improvements. Information is given for the wavelengths, energy levels, transition probabilities, Lande $g$-factors, and ionization energies of many atoms and ions. The Database now contains evaluated data for over 100,000 spectral lines and over 70,000 energy levels.

This new version is based on a relational database management system, which assures a high level of consistency and integration of the data, as, for example, for the information on the lines and levels of a particular atom. New interface features include instant access to bibliographic references, dynamic transition (Grotrian) diagrams, online generated plots for spectral line identification, and online generated plots for Saha/LTE plasma emission spectra for arbitrary electron temperature and density.

CONTACT: DR. JOSEPH READER (301) 975-3222 joseph.reader@nist.gov

\section{CODATA}

\section{Recommended Fundamental}

\section{Constants}

Based on work of the Fundamental Constants Data Center of the Division, the Committee on Data for Science and Technology (CODATA) has recommended for international use a new set of values of the fundamental physical constants and energy-related conversion factors.

The new set of values, termed the 2002 CODATA recommended values, became available to the public on December 8, 2003 at http://physics.nist.gov/constants. A detailed paper on the data selection and methodology of the adjustment is due to be published in the Reviews of Modern Physics. 
This review of the fundamental constants provides recommended values and their associated uncertainties, updating the last review of 1998 . Since then, new methods have become competitive for the determination of the Planck constant $h$, the fine-structure constant $\alpha$, and the relative atomic mass of the electron $m_{\mathrm{e}}$; and there has been a dramatic improvement in the measurement of the Newtonian constant of gravitation $G$.

Two noteworthy additions in the 2002 adjustment are recommended values for the bound-state RMS charge radii of the proton and deuteron, and tests of the exactness of the Josephson and quantum-Hall-effect relations $K_{\mathrm{J}}=2 e / h$ and $R_{\mathrm{K}}=h / e^{2}$, where $K_{\mathrm{J}}$ and $R_{\mathrm{K}}$ are the Josephson and von Klitzing constants, respectively, and $e$ is the elementary charge.

This work has met with immediate acceptance by the scientific community. The new values were published in the online August 2004 Physics Today Buyer's Guide and in the $85^{\text {th }}$ edition of the CRC Handbook of Chemistry and Physics for 2004-2005.

\section{CONTACT: DR. PETER J. MOHR (301) $975-3217$ peter.mohr@nist.gov}

\section{Precision Measurement of Reference Wavelengths for \\ 193 nm Lithography}

Some of the newest tools for microlithography are based on the ArF excimer laser, which emits radiation that can be tuned over a narrow band near $193 \mathrm{~nm}$. In order to design systems that focus the laser light onto substrate surfaces, lens designers must know the index of refrac-
Wavelength [nm]

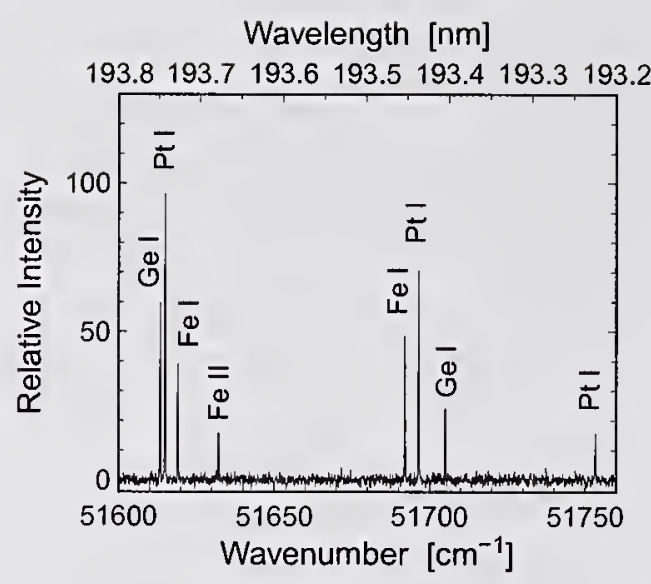

Figure 7. High-resolution spectrum of iron, germanium, and platinum, showing lines measured as wavelength standards for lithography at $193 \mathrm{~nm}$

tion of the optical materials to a high degree of accuracy. Since the index of refraction varies rapidly in this spectral region, it is essential that the $\operatorname{ArF}$ laser be stabilized at an accurately known wavelength.

In one method used to determine the laser wavelength, the laser is observed along with reference spectra of iron, germanium, and platinum, excited in hollow cathode discharge lamps. The ArF laser wavelength is determined by interpolation among the reference lines. However, until now the wavelengths of the reference lines were not known sufficiently well to obtain the desired accuracy for the laser wavelength.

By using a Fourier transform spectrometer optimized for use in this region of the ultraviolet, we have made precise wavelength measurements for seven lines of iron, germanium, and platinum that span the tuning range of the ArF laser. (See Fig. 7.) An overall relative uncertainty of $3 \times 10^{-8}$ was achieved. These results will serve to determine the $\mathrm{ArF}$ laser wavelength to the required accuracy. The experiment was conducted in collaboration with an excimer laser manufacturer that supplies lasers for microlithography applications, and the results have been incorporated in a commercial laser system.

CONTACT: DR. GILLIAN NAVE (301) $975-4311$

gillian.nave@nist.gov

\section{X-Ray Probes of Metal-Halide Discharge Lamps}

There are about one billion plasma light sources in service in the United States, consuming an estimated 2 exajoules ( 600 billion kilowatt hours) of electrical energy annually. These sources are principally fluorescent lamps and metalhalide discharge lamps. In the past, metal-halide lamps were used mainly for high-intensity lighting of large spaces. Now, because of their high brightness and energy efficiency, they are also being developed for interior lighting. As a result, there is growing interest in better understanding the processes that govern the operation of metal-halide lamps. Advances in understanding can lead to improved design rules, advanced production methods, and eventually, more energy-efficient lighting.

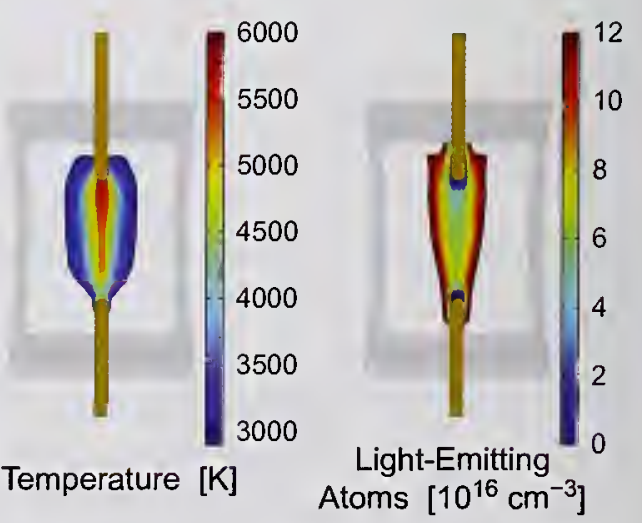

Figure 8. X-ray mapping of plasma properties in a metal-halide lamp. a) Temperature distribution from $x$-ray absorption imaging. b) Spatial distribution of atoms capable of emitting light from $\mathrm{x}$-ray induced fluorescence. 
We are developing new, noninvasive techniques to map the temperature and the spatial distribution of atomic and molecular constituents in metal-halide lamps. In experiments using the Advanced Photon Source (APS) at the Argonne National Laboratory, one of the world's most brilliant sources of $\mathrm{x}$ rays, we have observed $\mathrm{x}$-ray absorption and $x$-ray induced fluorescence in operating lamps. (See Fig. 8.) The use of $x$ rays rather than light allows investigation of advanced lamp designs having high-temperature ceramic envelopes that are translucent, in contrast to transparent quartz envelopes.

Some of these same techniques are now being developed on a smaller scale at NIST using a laboratory $\mathrm{x}$-ray source. This will make these new techniques more accessible to the lighting industry.

CONTACT: DR, JOHN J. CURRY

(301) 975-2817

john.curry@nist.gov

\section{Comprehensive Atomic}

\section{Spectral Data on Xenon and}

\section{its lons}

Reliable spectroscopic data for xenon and its ions are needed in order to understand processes taking place in many different types of electrical discharges, such as those used for EUV microlithography and the plasmas in new types of tokamaks. Although there are numerous data for xenon in the literature, they are scattered in a large number of publications and can be difficult to locate.
A critical compilation was assembled for the energy levels, spectral lines, and ionization energies of the xenon atom and all of its ions. This 157-page volume includes 1600 energy levels and 4800 observed lines. The critical compilation process collects the data, validates them by using various comparisons and theoretical calculations, and provides them in a form that is available in print as well as on the World Wide Web.

This compilation work was complemented by an experiment that revealed, for the first time, the detailed nature of the spectrum of ten-times ionized xenon at $13 \mathrm{~nm}$, the region of prime interest for EUV lithography. In this experiment, xenon gas was puffed into a high voltage spark, and the spectrum was observed with a high-resolution spectrograph.

CONTACT: DR, EDWARD B. SALOMAN (301) 975-5554

edward.saloman@nist.gov

\section{From Nanometers to} Megaparsecs: EBIT Data Applied to Disciplines

\section{Spanning 30 Orders of Magnitude}

With the recent addition of new measurement capabilities, the EBIT facility is attracting a wide range of customers who need atomic data from HCIs. At one extreme are the astrophysicists who are trying to resolve puzzles associated with observed line ratios in neon-like $\mathrm{Fe}$ (Fe XVII). X-ray lines from this ion are among the strongest to appear in many spectra from space observatories such as Chandra and XMM, but their utility as remote diagnostics is being hampered by inconsistencies across data sets.

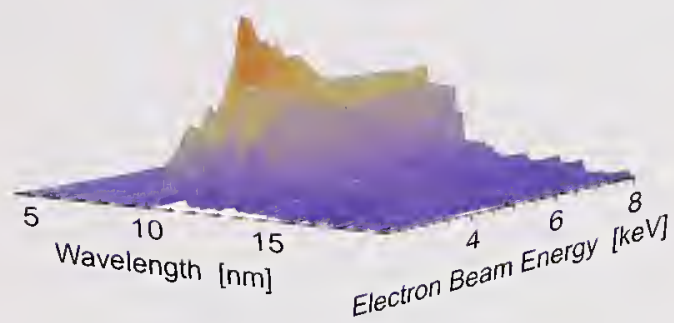

Figure 9. EUV spectra of xenon, taken on the NIST EBIT. The variation of spectral features as a function of the electron beam energy provides valuable data for benchmarking models of the atomic processes.

Benchmark Fe XVII data from our EBIT, previously reported in an Astrophysical Journal Letter, has been referenced several dozen times and has stimulated a number of independent experimental and theoretical studies elsewhere. In the meantime, we have installed a new $\mathrm{x}$-ray microcalorimeter, currently the best in the world by several measures, to address new issues raised by the recent theoretical work.

In a very different application, Intel and International SEMATECH have asked us to provide benchmark data on tentimes ionized xenon, the initial ion of choice for EUV lithography radiation sources. (See Fig. 9.) This work was stimulated by the needs of plasma modelers who are being employed by the semiconductor industry to ascertain fundamental limits on output power and to help achieve them. In connection with this work, the leader of the Plasma Radiation Group was appointed Chair of the International SEMATECH Fundamental Data Working Group and co-chair (with Intel) of the $1^{\text {st }}$ EUV Source Modeling Workshop.

CONTACT: DR. JOHN D. GILLASPY (301) 975-3236

john.gillaspy@nist.gov 


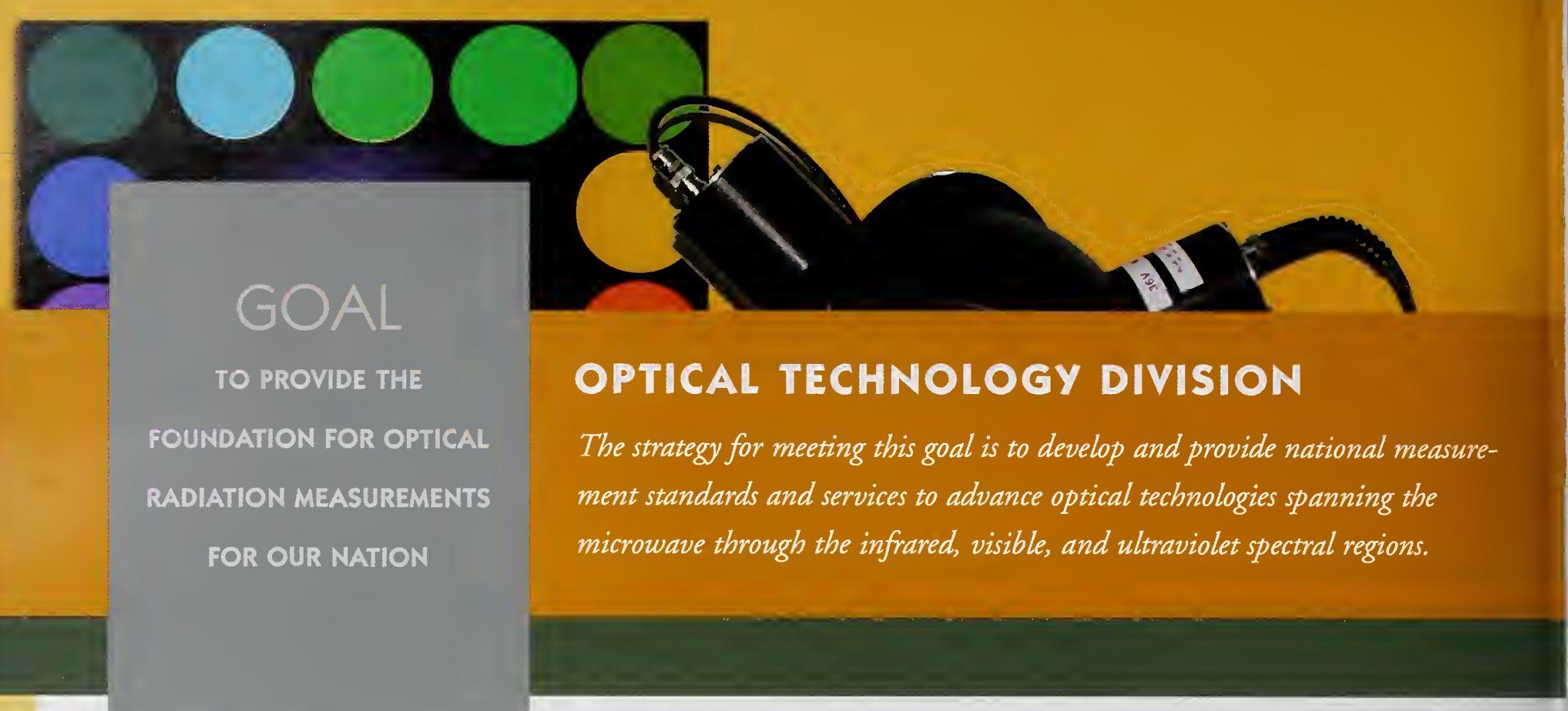

RADIATION MEASUREMENTS

FOR OUR NATION
The strategy for meeting this goal is to develop and provide national measurement standards and services to advance optical technologies spanning the microwave through the infrared, visible, and ultraviolet spectral regions.

\section{he first strategic}

element is to develop and

provide optical radiation

standards based on the

SI units.

\section{Optical Radiation Standards}

\section{INTENDED OUTCOME AND \\ BACKGROUND}

The Optical Technology Division plays a fundamental role in advancing the use of optical technology. Through research and development, we advance the measurement science needed to maintain the Nation's primary SI standards for the candela and kelvin, and associated photometric, colorimetric, pyrometric, and spectral radiometric quantities. These standards affect a host of industries, from aerospace to lighting, by ensuring the accuracy and agreement of measurements between and within organizations.

A significant part of the Division's activities includes participaring in international comparisons of measurements with other national metrology institutes. They are organized through the Consultative Committees on Temperature and on Photometry and Radiometry, of the International Committee of

Weights and Measures. These comparisons provide an important assessment of measurement quality, and help guarantee the international acceptability of our Nation's optical radiation measurements.

To ensure unsurpassed measurement accuracy, the Division's radiometric scales are increasingly being based on stable, low-noise, highly linear detectors, radiometers, and photometers. Their absolute responsivities are traceable to optical power measurements performed using cryogenic radiometry and a stateof-the-art laser facility, SIRCUS (Spectral Irradiance and Radiance Calibrations with Uniform Sources). Cryogenic radiometry provides the highest accuracy optical power measure- ments by performing a direct comparison of optical and electrical power. The Division's High-Accuracy Cryogenic Radiometer (HACR), the Nation's standard for optical power, achieves a relative standard uncertainty of better than $0.02 \%$.

The improvements realized by tying the radiometric measurements to cryogenic radiometry are significant. Recently the Division's spectral irradiance scale, as disseminated by FEL-type lamps, was converted to a detector-based scale traceable to the HACR. The improved scale has led to a reduction of spectral irradiance uncertainties by a factor of two in the ultraviolet and visible, and greater in the infrared. The spectral radiance and radiance temperature scales will also be detector based in the near future.

These advances in detector-based radiometry are complemented by new research in source-based radiometry, i.e., radiometry based on a source of radiation whose spectral output is absolutely known. This research uses synchrotron radiation from an electron storage ring, 
the recently upgraded NIST

Synchrotron Ultraviolet Radiation Facility (SURF III), as a source of continuously tunable, intense, ultraviolet light. The SURF III effort is complemented by programs to develop improved blackbody sources.

\section{AC COMPLISHMENTS}

\section{High-Temperature}

\section{Radiometric Standards}

To address the lack of high-temperature standards in the International Temperature Scale of 1990 (ITS-90), the Division is researching the suitability of blackbody-based standards tied to the fixed points of the phase transitions in metal-carbon and metal-carbide-carbon eutectics. Such fixed points offer the potential for temperature standards as high as $3034 \mathrm{~K}$ (using the TiC-C eutectic), over a factor of two greater than the present high-temperature limit of $1358 \mathrm{~K}$ (fixed at the Cu freezing point). The availability of high-temperature standards would allow routine measurements to be made between $1358 \mathrm{~K}$ and $3034 \mathrm{~K}$ by interpolation rather than extrapolation from the $1358 \mathrm{~K}$ standard, resulting in a reduction of temperature measurement uncertainties by a factor of five near $3000 \mathrm{~K}$.

To accurately measure the thermodynamic temperatures of these fixed points, the Division is developing absolute pyrometers calibrated against a cryogenic radiometer using SIRCUS. The first generation of these absolute pyrometers, denoted AP1 and operating at $650 \mathrm{~nm}$, has a noise figure of approximately $2 \mathrm{mK}$ and a one-year stability of approximately $100 \mathrm{mK}$ at $1300 \mathrm{~K}$. Efforts are directed at improving the stability and extending the operating wavelength into the ultraviolet near $365 \mathrm{~nm}$, to achieve reduced uncertainties near $3000 \mathrm{~K}$.

In assessing the appropriateness of these eutectics for high-temperature standards, the Division recently participated in a measurement comparison with the National Physical Laboratory (UK). The measured phase-transition temperatures were compared for five metal-carbon eutectics (Co-C, $1597 \mathrm{~K}$; Pd-C, 1765 K; Pt-C, 2011 K; Ru-C, $2226 \mathrm{~K}$; and $\mathrm{Re}-\mathrm{C}, 2748 \mathrm{~K}$ ) relative to the ITS-90 temperature scale. The measured temperatures agreed to within $0.5 \mathrm{~K}$, demonstrating the utility of these eutectics for international temperature standards.

\section{CONTACT: DR. HOWARD W. YOON (301) 975-2482 \\ howard.yoon@nist.gov}

\section{New Infrared Calibration}

\section{Facility}

The increased need for highly accurate infrared radiation measurements for homeland security, national defense, and remote sensing applications has led the Division to develop, with support from the Air Force through the Department of Defense Calibration Coordination Group (CCG), an infrared SIRCUS facility.

When completed, IR-SIRCUS will extend the visible-to-ultraviolet capabilities of the present SIRCUS into the $1 \mu \mathrm{m}$ to $20 \mu \mathrm{m}$ wavelength region of the infrared, including the critical longwave and mid-wave infrared atmospheric windows, coincident with the peak spectral responsivities of $\mathrm{HgCdTe}$ and InSb single-element detectors and focal plane arrays. (See, e.g., Fig. 1.)

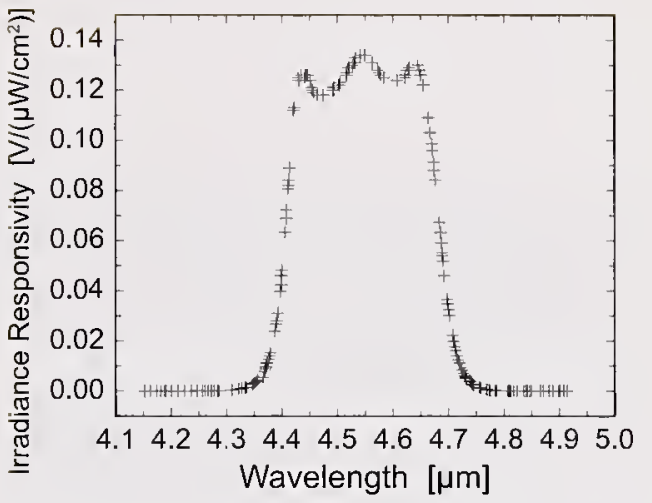

Figure 1. Spectral irradiance responsivity for an InSb radiometer measured on IR-SIRCUS.

The facility will allow the characterization and calibration of a variety of infrared imaging and camera systems, including hyperspectral imagers, at a level of detail and accuracy not possible with conventional broadband, blackbody radiation sources.

Challenging the development of IRSIRCUS is the lack of availability of commercial high-dynamic-range transfer detectors and broadly and continuously tunable high-power infrared lasers. Therefore, the IR-SIRCUS team has constructed a $1 \mu \mathrm{m}$ to $5 \mu \mathrm{m}$ infrared laser system based on optical parametric oscillators $\left(\mathrm{LiB}_{3} \mathrm{O}_{5}\right.$ (LBO-OPO) or periodically poled $\mathrm{LiNbO}_{3}$ (PPLN-OPO)) pumped by a mode-locked $\mathrm{Nd}$ :Vanadate laser. For a transfer-standard detector the team is using a NIST-developed, liquid-helium cooled, electricalsubstitution bolometer.

With this laser system and detector, infrared detectors, radiometers, and focal plane arrays can be calibrated on IR-SIRCUS for spectral power, spectral irradiance, or spectral radiance responsivity between $1 \mu \mathrm{m}$ and $5 \mu \mathrm{m}$ with a relative standard uncertainty of $1.5 \%$.

CONTACT: DR. GEORGE P. EPPELDAUER (301) $975-2338$

george.eppeldauer@nist.̧ov 


\section{Novel LED Sources for Radiometry}

The Division is developing new radiometric applications for light emitting diodes (LEDs) to take advantage of their unique properties, which include narrow bandwidth, high spectral brightness, compact size, high efficiency, and low cost.

A prototype LED-illuminated integrating sphere source has been successfully developed using 40 LEDs operating in 10 distinct bands between $380 \mathrm{~nm}$ and $780 \mathrm{~nm}$. Varying the light intensity in the individual bands allows variation of the spectral output or color, as shown in Fig. 2. A second-generation LED source, now under development, will use 150 diodes grouped into 40 distinct bands to achieve a source that can match a conventional source within $2 \%$ for luminance and 0.002 in chromaticity.

A compact $660 \mathrm{~nm}$ LED source, consisting of a tube containing 36 diodes illuminating a volume-diffuser output coupler, has also been developed to allow the routine monitoring of the sizeof-source error in optical pyrometers. The size-of-source error provides a rapid assessment of the stability of the optical alignment of the pyrometer and the surface cleanliness of the optics. It can be an important correction to the measurement and an important component of the measurement uncertainty, depending upon its magnitude.

Following a similar design, a compact, blue LED source has been developed to model ocean color for the calibration of instruments measuring ocean optical properties. By matching the spectral

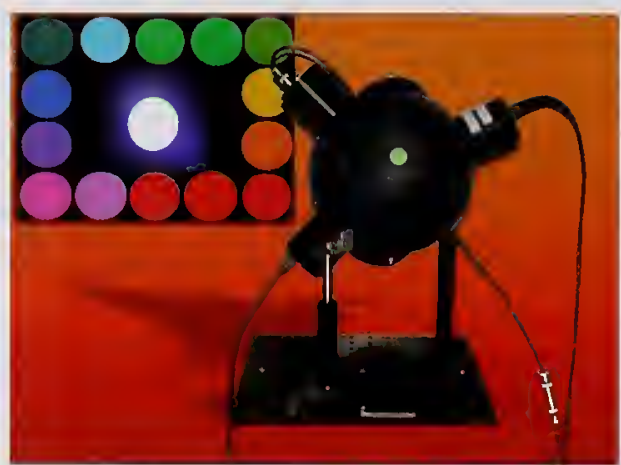

Figure 2. Solid-state integrating sphere source pumped by light emitting diodes, showing some of the different colors that can be senerated by varying the input current to the individual LEDs.

output to the ocean waters of interest, many potential sources of calibration error in the instrument under test, such as stray light, can be reduced or eliminated.

CONTACT: DR. STEVEN W. BROWN

(301) 975-5167

steven.brown@nist.gov

he second strategic

element is to develop

novel optical measurement

methods for solving problems

in critical and emerging

technology areas.

\section{Optical Measurement Methods}

\section{INTENDED OUTCOME AND \\ BACKGROUND}

The Division strives to improve the accuracy, acceptability, and utility of optical measurements by conducting long-term, directed research in the
NIST strategic focus areas of homeland security, nanotechnology, and health care, and in such promising technology growth areas as interface science, biophysics, semiconductor manufacturing, and quantum communication.

In the areas of nanotechnology and interface science, we are developing linear and nonlinear laser and near-field techniques to characterize thin films, surfaces, and interfaces important in molecular biology, polymer science, and nanotechnology.

In biophysics and health care, new optical methods based on surface-enhanced Raman spectroscopy and confocal, fluorescent imaging microscopy are being developed to investigate single biological molecules. This is part of a larger, interlaboratory competence program in Single-Molecule Measurement and Manipulation, funded by the NIST Director.

The Division's activities in support of semiconductor manufacturing include research to improve the accuracy of temperature measurements during processing, through better knowledge of the spectral emissivity of coated silicon wafers; to advance laser optical-scattering metrology, for next-generation wafer inspection tools; and to revolutionize optical microscopy for quality control in semiconductor manufacturing, by using interferometry to improve the spatial resolution, increase the throughput, and reduce the cost. Ultraviolet radiation metrology is being developed to ensure a measurement infrastructure for $157 \mathrm{~nm}$ and shorter wavelength lithography, leveraging the unique ultraviolet radiometry capabilities of SURF III to 
target needs in optical-materials properties and detector radiationdamage characterization.

In response to national priorities, the Division continues to research optical radiation measurements for homeland security and national defense. Optical measurements and standards are being developed for missile defense, sensor calibration, target identification, and night vision. The Division has built specialized laboratories, such as the LowBackground Infrared (LBIR) Facility and IR-SIRCUS, to help support these efforts. Building upon expertise developed through the NIST Director's competence program in $\mathrm{THz}$ Metrology, the Division is investigating continuous wave and femtosecond-pulsed $\mathrm{THz}$ spectroscopy and imaging for the detection of chemical and biological warfare agents. The Division is also using its unique expertise in correlated photon radiometry to develop novel singlephoton-on-demand sources for secure quantum cryptography, in collaboration with other Divisions.

\section{ACCOMPLISHMENTS}

\section{Aperture Area Determinations}

\section{for Satellite Measurements}

The Division is providing measurements of the areas of precision optical apertures to improve the accuracy of satellite exoatmospheric solar-irradiance measurements, critical for assessing the absolute magnitude of the approximately $1366 \mathrm{~W} / \mathrm{m}^{2}$ solar radiation driving Earth's weather and climate. Presently, independent satellite exoatmospheric solar-irradiance measurements suffer from systematic discrepancies of approximately $0.6 \%$ over the last two decades, reduced to $0.1 \%$ for the last decade. The relative uncertainty of the aperture area measurement places a lower limit on the relative uncertainty of the irradiance scale.

It is anticipated that the recent launch of the SOlar Radiation and Climate Experiment (SORCE), in January 2004, will improve solar irradiance measurements through better instrumentation and increased efforts to establish traceability of the measurements to national scales maintained by NIST. For the Total Irradiance Monitor and the Spectral Irradiance Monitor aboard the SORCE satellite, NIST has provided accurate measurements of the areas of the apertures for the irradiance measurements, and has modeled the diffraction corrections associated with transmission through the apertures.

The NIST measurements were performed at the Aperture Area Measurement Facility. The facility uses a coordinate measuring machine to ascertain selected edge points of the aperture, as located by a video microscope. Distances are measured interferometrically, yielding aperture areas with typical relative standard uncertainties $(k=1)$ of approximately $2.5 \times 10^{-5}$.

The Division is also leading a comparison of aperture area measurements among laboratories involved in the development of exoatmospheric solarirradiance sensors. Its main goal is to assess whether inaccurate aperture area determinations are the origin of the discrepancy in reported solar irradiances. The comparison, which so far includes two other laboratories, indicates that aperture area measurements account for $<0.1 \%$ of observed variation among sensors.

CONTACT: DR. MARITONI LITORJA (301) $975-8095$ maritoni.litorja@nist.gov

\section{Workshop on Satellite Instrument Calibration}

In January 2004, the Division released its final report from the workshop, Satellite Instrument Calibration for Measuring Global Climate Change, held in November 2002 and jointly organized by NIST through the Optical Technology Division, the National Polar-orbiting Operational Environmental Satellite System-Integrated Program Office, NOAA, and NASA.

This workshop was initiated to address the measurement challenges and instrument calibration needs for the accurate space-based measurement of global climate-change variables such as atmospheric temperature and solar irradiance. These variables are critical for assessing the magnitude of, and determining an effective policy response to, global climate change.

The NIST report, NISTIR 7047, summarizes the required absolute accuracies and stabilities of climate-change variable determinations for use in predictive climate-change models. The report relates required climate-change variable accuracies and stabilities to satellite measurement accuracies and stabilities. The report also assesses the ability of present satellite technology to deliver the required measurement accuracy and stability. Guiding principles to maintain and improve the accuracy of satellite 


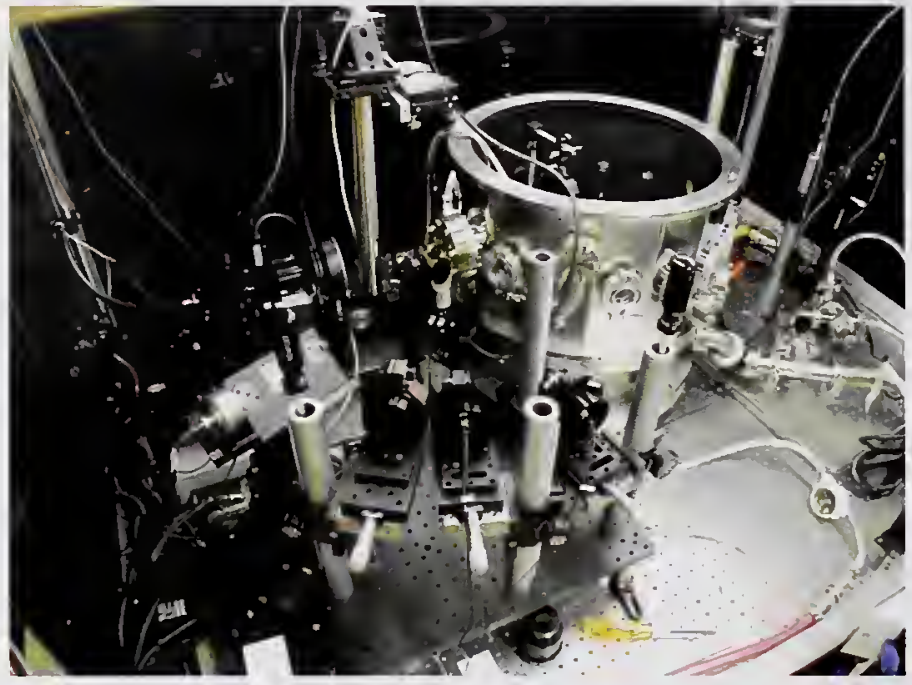

Figure 3. Vacuum goniometer for the measurement of the spectral emittance of specular samples as a function of temperature and angle.

remote-sensing measurements, through better instrument calibration and validation, are also provided in the report. Finally, the report ends with a proposal for a follow-up workshop to develop an implementation plan for the recommendations. Such a follow-up workshop is planned in the near future.

\section{CONTACT: DR. RAJU V. DATLA}

(301) 975-2131

raju.datla@nist.̧ov

\section{Emittance Measurements for}

\section{Semiconductor Manufacturing}

A new facility has been developed to determine the emittance of silicon wafers as a function of temperature, wavelength, and angle, in order to improve temperature measurements during semiconductor manufacturing. The emittance of a material means how well it emits optical radiation relative to an ideal, blackbody radiator. Knowledge of the emittance of a material is essential for accurate noncontact temperature measurements based on Planck's Radiation Law. Such measurements are performed in support of many industrial

\section{Improved Ocean-Color Measurements}

To improve satellite ocean-color measurements used to estimate oceancarbon levels, Division scientists have undertaken a detailed optical characterization of the Marine Optical Buoy (MOBY), permanently stationed off the coast of Hawaii. MOBY uses dual imaging spectrographs to provide measurements of ocean-leaving radiances and downwelling radiances. These data are compared to simultaneous measurements performed by overflying satellites such as the Sea-viewing Wide Field-ofview Sensor (SeaWiFS) and the Moderate Resolution Imaging Spectroradiometer (MODIS), thus providing vicarious calibration of the satellite measurements.

The motivations for the characterization were the discrepancy between MOBY's two spectrographs (denoted red and blue) in their overlap region and the unphysical, negative leaving radiance observed at short, near-ultraviolet wavelengths in the blue channel. To undertake the characterization, Division scientists developed Traveling SIRCUS for the off-site characterization of the spectral responsivity of the instrument, using a tunable-laser-illuminated integratingsphere source.

The measurements demonstrated that stray light within the spectrographs from high-order grating reflections and scattered light caused the problems. A correction algorithm was developed that removed the spectral artifacts caused by the stray light. The corrections due to stray light alone have led to a lowering of the SeaWiFS satellite measurements of global chlorophyll a concentrations, and associated ocean-carbon levels, by 
approximately $15 \%$ to $20 \%$. When combined with other readjustments, the values were approximately $6 \%$ lower than those previously accepted.

CONTACT: DR. CAROL JOHNSON

(301) 975-2322

cjohnson@nist.gov

\section{Coherent Control Explored in the Condensed Phase}

The Division, in collaboration with the Max Planck Institute in Garching, Germany, has investigated the molecular response following amplitude- and phase-controlled femtosecond midinfrared multiphoton excitation of a molecular stretching vibration. In this case, the carbonyl stretch in tungsten hexacarbonyl, $\mathrm{W}(\mathrm{CO})_{6}$, was studied. Such coherent-control methods offer the potential of directly controlling intermolecular and intramolecular energy-transfer processes and chemical reactions, through the optimal preparation of vibrationally excited precursor molecules.

$\mathrm{W}(\mathrm{CO})_{6}$ in hexane solution was selected as a test case because its $\mathrm{CO}$-stretching fundamental has both a large transition dipole moment, necessary for a high quantum yield, and a large vibrational anharmonicity, allowing resolution of individual $v+1 \leftarrow v$ vibrational hotband transitions. In addition to measuring the anharmonic shifts and linewidths of the $v+1 \leftarrow v$ progressions, controlled population distributions were compared to Bloch model calculations for the excitation process.

The main result, presented in Fig. 4, shows differential absorption for vibrationally excited $\mathrm{W}(\mathrm{CO})_{6}$ in room temperature $n$-hexane as a function of probe frequency. A strong ground-state bleach

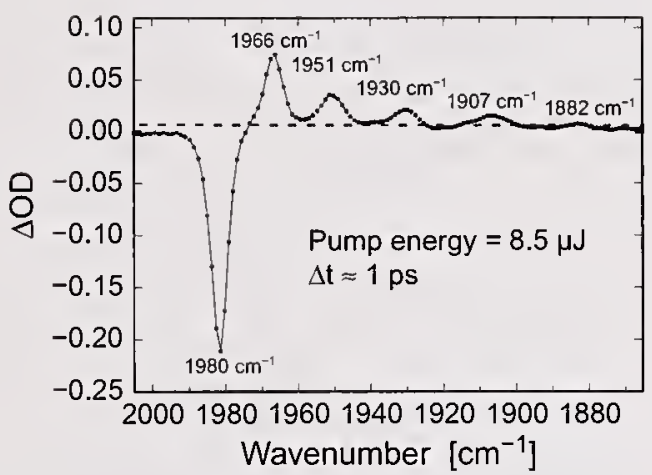

Figure 4. Spectrum showing differential absorption for vibrationally excited $W(\mathrm{CO})_{6}$ in room-temperature $n$-hexane as a function of probe frequency. A strong ground-state bleach signal at $1980 \mathrm{~cm}^{-1}$ is accompanied by five new absorption bands, from the first five $v+1 \leftarrow v$ hotband transitions.

signal at $1980 \mathrm{~cm}^{-1}$ is accompanied by five new probe absorption bands from the first five $\Delta v=1$ hotband transitions. Probe absorption at $1882 \mathrm{~cm}^{-1}$, arising from the $v=6 \leftarrow 5$ transition, implies population transfer to $v=6$, corresponding to an energy of nearly $12,000 \mathrm{~cm}^{-1}$ deposited into a single vibrational mode. About $0.5 \%$ of the molecules are excited to the dissociation energy limit for a W-C bond.

Future experiments will attempt to induce ground-electronic-state molecular bond dissociation in the condensed phase. Planned extensions of this study include applying feedback to the amplitude and phase modulated MIR laser pulses to increase the energy deposited into the vibrational mode.

CONTACT: DR. EDWIN J. HEILWEIL

(301) 975-2370

edwin.heilweil@nist.gov

\section{Terahertz Spectroscopy of Polypeptides}

The continuous wave terahertz spectra of small polypeptides have been measured to better understand the lowfrequency, large-amplitude vibrations responsible for the flexibility of biological molecules.

Tunable $\mathrm{THz}$ radiation was generated by the solid-state photomixing of two near-infrared laser beams separated in frequency from $0.1 \mathrm{THz}$ to $3 \mathrm{THz}$. Samples of a polypeptide mixed in a transparent polytetrafluoroethylene matrix or dissolved in an aqueous solution were studied from $4.2 \mathrm{~K}$ to $300 \mathrm{~K}$. Spectra were recorded for the series of polypeptides from alanine to hexa-alanine, in different crystalline forms.

Variations in the spectra with crystal structure, hydration level, and temperature reveal the importance of intermolecular hydrogen bonding and vibrational anharmonicity in controlling the dynamics of the low-frequency vibrational modes. The positions of the absorption peaks change dramatically with water hydration level due to perturbations of the vibrational force field from hydrogen bonds between the peptide and neighboring water molecules in the matrix. A decrease in the apparent vibrational anharmonicity with decreasing temperature leads to a narrowing of the absorption peaks and a decrease in the asymmetry of the lines' profiles.

Efforts at modeling these effects are underway, to allow the extraction of quantitative information about the inter- and intramolecular force fields of these model polypeptides.

CONTACT: DR. DAVID F. PLUSQUELLIC

(301) 975-3896

david.plusquellic@nist.gov 


\section{he third strategic}

element is to disseminate

optical radiation measure-

ments and standards to

industry, government,

and academia.

\section{Optical Measurement}

\section{Services}

\section{INTENDED OUTCOME AND \\ BACKGROUND}

The Division builds and maintains world-class optical radiation measurement facilities to meet the continued and emerging needs for standards and specialized measurements by government and industry. These facilities are available to government and industry customers through formal calibration services, special tests, and standard reference materials available from NIST Technology Services, or through collaborative research efforts.

The Division maintains facilities for measuring optical properties such as reflectance, retroreflectance, transmittance, color, and gloss; for photometric measurements such as luminous intensity and color temperature; and for radiometric measurements such as spectral radiance, spectral irradiance, spectral power, detector responsivity, and radiance temperature. The Division has highly specialized facilities for performing low-background radiometric measurements, for characterizing remote sensing instruments, for measuring the area of precision radiometric apertures, and for determining the absolute optical power, radiance, and irradiance spectral responsivities of instruments. New measurement facilities are under development to measure the emittance of materials and to provide standards for ultraviolet spectral irradiance.

The Division strives to ensure the quality of these programs by publishing our research and measurement methodologies as NIST Special Publications or in outside peer-reviewed archival journals; by participating in measurement comparisons with other laboratories; and by maintaining a measurement quality program. The Division also aids the dissemination of good optical-radiation measurement practice by publishing training documents and by offering formal short-courses in photometry, spectroradiometry, and radiation temperature measurement. We are enlarging this activity to provide training at customer sites when the demand is large and resources are available.

\section{ACCOMPLISHMENTS}

\section{New LED Measurement}

\section{Services Launched}

The Division's conventional measurement services for the lighting industry have been optimized for standard incandescent and fluorescent lighting, and thus do not encompass the unique spatial and spectral distributions of LEDs. However, the Division has developed several new measurement services for LEDs, providing critical standards necessary for our Nation to rapidly realize the significant energy and cost savings, and greenhouse-gas reductions, expected for solid-state lighting-and to become a commercial leader in this new technology.
New measurements services include the calibration of photometric quantities such as averaged LED luminous intensity in candelas and total luminous flux in lumens, and of colorimetric quantities such as chromaticity coordinates and dominant wavelength. (See Fig. 5.) Uncertainties $(k=2)$ of $1 \%$ to $3 \%$ for averaged LED luminous intensity, $1 \%$ to $2 \%$ for luminous flux, and 0.001 in $(x, y)$ chromaticity coordinates have been achieved.

These measurement services are necessary tools for industry to assess the performance of LEDs and to ensure consistent measurements between manufacturers. The colorimetric standards will also help industry to optimally blend combinations of LEDs to achieve the color rendering demanded by users.

Future efforts will include developing approaches for the radiometric calibration of LEDs in the ultraviolet and near-infrared spectral regions.

\section{CONTACT: MR. YUQIN ZONG}

(301) 975-2332

yuqin.zong@nist.gov

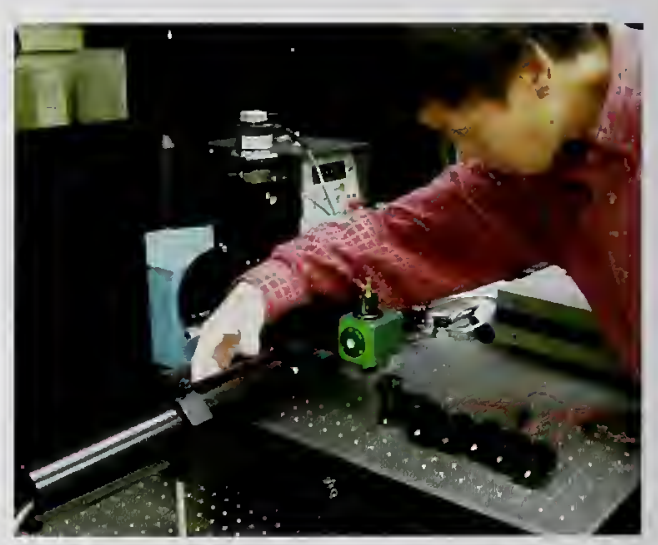

Figure 5. Yuqin Zong aligns a green LED for calibration on the reference spectroradiometer 


\section{Center for High-Accuracy}

\section{Retroreflection}

\section{Measurements (CHARM)}

The Division has recently completed development of a new facility to measure the retroreflectivity of pavement markings and road signs, with financial support from the National Cooperative Highway Research Program. The facility was developed in response to a Congressional directive to the U.S. Department of Transportation to establish "a standard for a minimum level of retroreflectivity that must be maintained for pavement markings and signs which apply to all roads open to public travel."

The requirements for the NIST reference retroreflectometer were based on a careful examination of national and international documentary and artifact standards, and discussions with experts. The facility is installed in a $40 \mathrm{~m}$ long tunnel, and consists of a six-axis goniophotometer, a uniform beam source, and a detector system mounted on $35 \mathrm{~m}$ long rails. (See Fig. 6.)

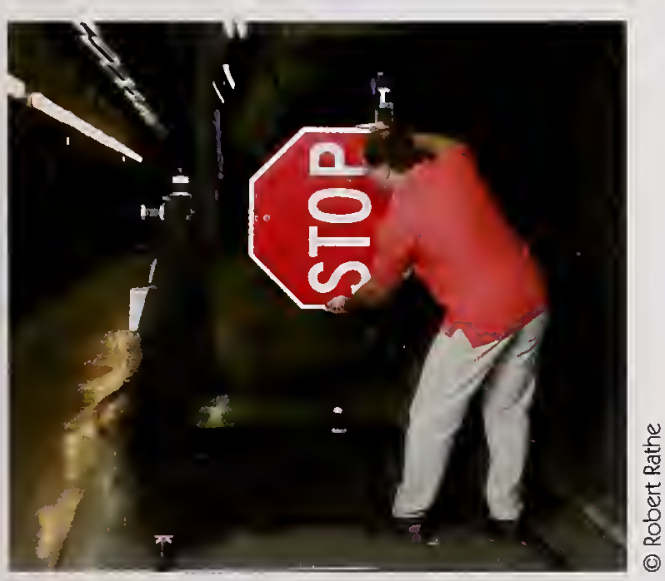

Figure 6. Cameron Miller mounts a STOP sign on the goniometer at the NIST Center for High-Accuracy Retroreflection Measurements (CHARM).
The instrument has been fully characterized, and a complete uncertainty analysis has been performed. A calibration of typical retroreflective sheeting material for coefficient of luminous intensity will yield a relative expanded uncertainty of $1 \%(k=2)$. Typical pavement marking materials will have a slightly higher relative expanded uncertainty of approximately $2 \%(k=2)$. An official calibration service for retroreflectance materials began in 2004.

\section{CONTACT: DR. CAMERON MILLER (301) 975-4713 \\ c.miller@nist.gov}

\section{LBIR Facility Aids Missile Defense}

Recent upgrades in the LBIR Facility have improved the Division's ability to provide infrared measurements and standards critical to the development and implementation of the U.S. National Missile Defense (NMD) system. This work supports the mission of the Ballistic Missile Defense Organization (BMDO) by helping to ensure that the infrared sensors mounted on interceptor missiles can reliably discriminate between target and decoy.

The LBIR Facility uses specialized radiometric test chambers and radiometers to disseminate the NIST infrared radiance scale to Government and contractor sites, by providing on-site calibration of blackbodies or off-site calibration of test chambers. Upgrades to the LBIR Facility included improvements in the cryogenic system to allow operation down to $15 \mathrm{~K}$ (the previous system limit was $25 \mathrm{~K}$ ), and the addition of a low-noise absolute cryogenic radiometer. Together these improve- ments have led to a factor-of-ten reduction in the measurement uncertainty for on-site blackbody calibrations.

Improvements have also been made in the ability to provide off-site calibration of cryogenic test chambers using the $2 \mu \mathrm{m}$ to $30 \mu \mathrm{m}$ BMDO Transfer Radiometer (BXR I). The BXR I has been sent to space test chambers at Boeing, Raytheon, and the Air Force to tie measurements performed at these sites to the NIST High-Accuracy Cryogenic Radiometer. Improvements in the calibration of the BXR I radiome-

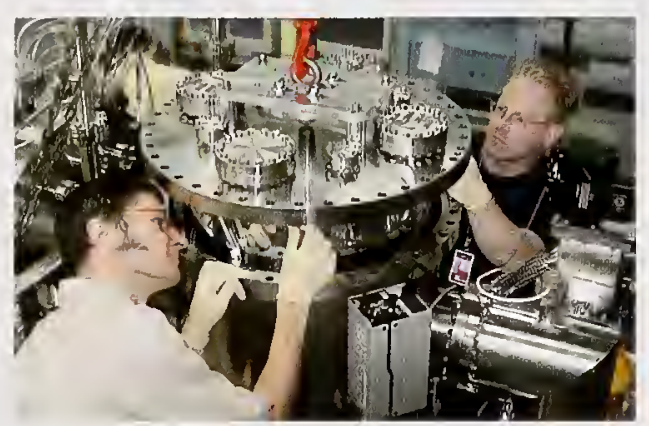

Figure 7. James Fedchak and Allan Smith install the new $10 \mathrm{~cm}$ collimator in the LBIR Facility to reduce the uncertainty in the calibration of the $\mathrm{BXR}$ I transfer-standard radiometer.

ter at NIST are being undertaken by using a specially designed $10 \mathrm{~cm}$ light collimator to better simulate the performance of the space chambers calibrated in the field by the BXR I (Fig. 7). Implementation of this collimator in the calibration of the BXR I at NIST is expected to provide a three-fold improvement in the measurement uncertainty at the NMD space chambers. Such improvements are essential if infrared sensors are to meet future NMD accuracy requirements.

CONTACT: DR. ADRIAAN CARTER

(301) 975.4765

adriaan.carter@nist.gov 
methods based on mass spectrometry; and 4) a national quality assurance system that supports homeland security needs.

Many tens of thousands of low-level radiochemical measurements are made annually to support environmental remediation and occupational health programs. The credibility of these measurements has been based on participation in regulation-driven performance evaluation programs of limited scope. The metrology community recognized a fundamental flaw-that there was often a lack of direct linkage to national radioacrivity standards. This situation is being addressed by the publication of three ANSI standards. These consensus standards call for a traceability-testing program that links the quality of operational measurements to national standards.

\section{ACCOMPLISHMENTS}

\section{Testing of Radiation}

\section{Detection Portable and Portal}

\section{Instruments}

Characterizations of commercially available instruments for measurement and identification of unknown radionuclides were carried out in support of the development and testing of American National Standards Institute (ANSI) standards N42.32, N42.33, N42.34, and N42.35. Measurements were based on the performance of the devices, i.e., the capability of the detectors to ensure a correct radionuclide identification and to determine exposure or dose-rate values, in a given time interval, for various radioactive sources and accuracies.
Thirty portable instruments and four portal monitors were tested. The portable instruments' responses, as a function of energy, were tested using two ${ }^{60} \mathrm{Co}$ and two ${ }^{137} \mathrm{Cs}$ ranges, as well as the $\mathrm{x}$-ray range operating at effective energies of $47 \mathrm{keV}, 80 \mathrm{keV}$, and $120 \mathrm{keV}$.

For portal monitors, a new testing facility was installed, allowing the testing of these monitors using several radioactive sources transported through the monitors at different speeds and distances. The monitors were field-tested in a parking lot and at the NIST C Gate. The availability of this new testing facility and our access to a wide variety of radioactive sources allow us to test portal monitors presently being developed for detection and identification of radionuclides.

\section{CONTACT: DR. LETICIA PIBIDA}

(301) 975-5538

leticia.pibida@nist.gov

\section{Gamma-Ray Emitting Test}

\section{Sources for Portal Monitors}

\section{used for Homeland Security}

Basing our research on the new ANSI N42.35 standard, "Evaluation and Performance of Radiation Detection Portal Monitors for Use in Homeland Security," as well as the related Test and Evaluation Protocol for Homeland Security, we developed a set of test sources for the testing of portal monitors. (See Fig. 2.)

The standard specifies that the test sources should be point sources (relative to the size of the portal monitors), single radionuclides, and encapsulated in $0.25 \mathrm{~mm}$ thick stainless steel disks. The

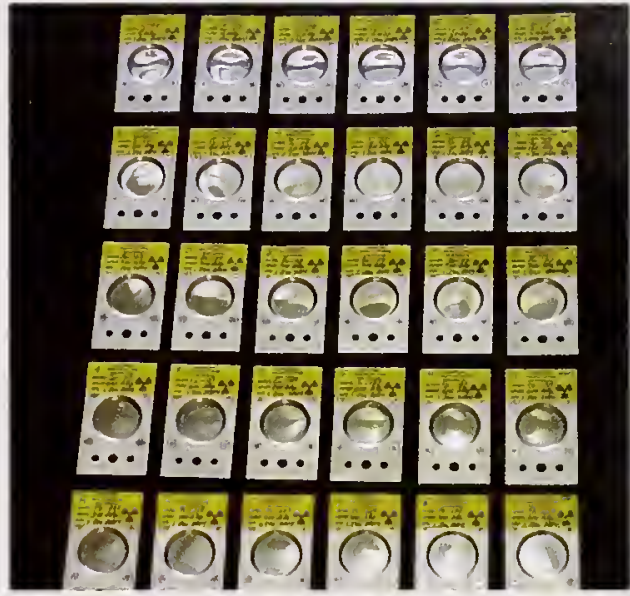

Figure 2. Radioactive test sources developed at NIST for the testing and evaluation of radiation detectors to be used by first responders.

radionuclides required for portal monitor testing are ${ }^{57} \mathrm{Co},{ }^{60} \mathrm{Co},{ }^{133} \mathrm{Ba},{ }^{137} \mathrm{Cs}$, ${ }^{228} \mathrm{Th}$, and ${ }^{241} \mathrm{Am}$. These sources are useable not only for testing and evaluating instrumentation, but also for calibrating test sources and for training instrument users.

We are aware of five companies that currently produce or distribute radioactive sources in the USA. AEA Technology showed an interest in commercializing our test sources, and they are getting ready to produce and distribute them more widely.

AEA Technology participates in a Measurement Assurance Program with NIST to assure traceability to U.S. national standards for sources used in nuclear power and medical applications. A similar and more extended program is being implemented for homeland security applications.

CONTACT: DR. LETICIA PIBIDA

(301) 975-5538

leticia.pibida@nist.gov 


\section{Microcalorimetry for Absolute}

\section{Radioactivity Standardizations}

A commercial "isothermal micro-

calorimeter" has been adapted for use in performing classical, calorimetric-based calibrations of radioactive sources.

Microcalorimetry is one of the best nondestructive methods for the standardization of small, high-activity (GBq-range), $\beta$ and $x$-ray sources, a category under which almost all brachytherapy sources fall.

This dual-cell, near-isothermal (heat flow) microcalorimeter operates at nearambient temperatures, utilizing specially fabricated, source-holder measurement cells that are used to maximize the energy absorption of the ionizing radiation. It incorporates resistance heaters within the measurement cells to very accurately determine power calibrations.

In 2004, to improve the temperature stability of the operating environment, the isothermal microcalorimeter was moved into the new AML building. The instrument is now located in a laboratory with ambient temperature stabilized to $0.2{ }^{\circ} \mathrm{C}$, which has increased the stability of the instrument baseline, and thus its sensitivity, by a factor of two.

The isothermal microcalorimeter was used for the calibration of an ${ }^{55} \mathrm{Fe}$ lowenergy $x$-ray source. The low energies of the emitted $x$ rays and Auger electrons (less than $6.5 \mathrm{keV}$ ) made use of conventional counters to measure this radionuclide extremely difficult. On the other hand, the low energy of the decay particles allowed for the primary calibration of ${ }^{55} \mathrm{Fe}$ by microcalorimetry without the need for an additional absorber within the cell, thus increasing sensitivity.

Preliminary results suggest that the standardization of ${ }^{55} \mathrm{Fe}$ sources by isothermal microcalorimetry can be performed with an expected accuracy of better than $1 \%$.

CONTACT: DR. PETER VOLKOVITSKY (301) 975-5527

peter.volkovitsky@nist.gov

\section{Measurement Standards for}

\section{Recently Approved}

\section{Radiotherapy Nuclide}

The American Cancer Society estimates that there will be 64,000 new cases of lymphoma in the U.S. this year, with an expected five-year survival rate (for nonHodgkin's lymphoma) of $52 \%$. One of the most promising new drugs for treatment of this disease is Zevalin, which is a monoclonal antibody labeled with the radionuclide ${ }^{90} \mathrm{Y}$. While ${ }^{90} \mathrm{Y}$ has many properties that make it suitable for use in radiotherapy, those same properties present a number of challenges regarding its measurement.

FDA approval of Zevalin brought about an exploding need for standards and measurement quality assurance for radiopharmaceuticals using ${ }^{90} \mathrm{Y}$. A workshop held at NIST in December 2001 identified the measurement issues involved. Invited speakers included representatives from NIH, radiopharmaceutical manufacturers, radiopharmacies, isotope producers, government regulators, and NIST.
As a result, NIST examined the measurement of ${ }^{90} \mathrm{Y}$ solution standards in clinically useful geometries, to develop calibration factors and an estimation of the uncertainties involved in those measurements. NIST worked with radiopharmacies, the FDA, the Society of Nuclear Medicine, the American Pharmaceutical Association, and others to organize and implement a protocol to establish measurement traceability for the estimated 450 radiopharmacies.

In related work, NIST assisted in the development of a long-term standard for the calibration of detectors used in ${ }^{90} \mathrm{Y}$ measurements. The short half-life $(64.053 \mathrm{~h})$ of ${ }^{90} \mathrm{Y}$ does not allow for long-term use of a single standard source. However, AEA Technology QSA (Burlington, Massachusetts) manufactures a mock ${ }^{90} \mathrm{Y}$ syringe using a solid source of the long-lived parent radionuclide ${ }^{90}$ Sr. NIST examined the characteristics of these sources in a detector, as compared to those of ${ }^{90} \mathrm{Y}$ solution sources. With the proper execution of an initial calibration exercise at a clinic, these standards may be used to check calibration throughout the year.

CONTACT: MR, JEFFREY T. CESSNA (301) 975-5539 jeffrey.cessna@nist.gov 
he second strategic

element is to develop and

provide neutron standards

and measurements needed

for fundamental physics,

homeland security, the

hydrogen economy, worker

protection, and nuclear

power.

\section{Neutron Standards}

\section{and Measurements}

\section{INTENDED OUTCOME AND}

BACKGROUND

The Neutron Interactions and Dosimetry Group provides measurement services, standards, and fundamental research in support of NIST's mission as it relates to neutron technology and neutron physics. The national interests served include industrial research and development, national defense, homeland security, higher education, electric power production, and more specifically, neutron imaging, scientific instrument calibration and development, neutron source calibration, detection of concealed nuclear materials, radiation protection, and nuclear and particle physics data.

The Group maintains and disseminates measurement standards for neutron dosimeters, neutron survey instruments, and neutron sources. The Group has initiated an accelerated program to develop effective neutron interrogation and measurement standards for national defense and homeland security needs, in collaboration with the Radioactivity Group.

The Group maintains and supports the Nation's premier fundamental neutron physics user facility. The Neutron Interferometry and Optics Facility is one of the most advanced user facilities of this kind in the world. The Group's neutron interferometry program provides the world's most accurate measurements of neutron coherent scattering lengths, important to materials science research and modeling of the nuclear potentials. Preparations for new neutron interferometry experiments for surface studies of multilayers, for determination of the charge distribution in the neutron, and for investigation of quantum information/coherence phenomena are being carried out.

The Group is at the forefront of basic research with neutrons in the measurements of symmetries and parameters of the weak nuclear interactions, addressing fundamental issues that are important in the understanding of theories of evolution of the cosmos. It is an internationally renowned program that maintains an extensive level of cooperation with premier national and international academic and research institutions.

We have developed and we maintain the Nation's only high-resolution neutron imaging user facility. It is available for both proprietary and open research at the NIST Center for Neutron Research (NCNR). Research at this facility is primarily dedicated to applying neutron imaging methods for industrial research on water transport in fuel cells and on hydrogen distribution in hydrogen storage devices. Neutron imaging, vigorously pursued by the Group, has rapidly developed to be the technique of choice for nondestructive, in situ water transport studies in PEM-type fuel cells. This facility has provided critical services to major automotive and fuel cell companies, and continues to be in high demand.

The Group is also among the world leaders in developing ${ }^{3} \mathrm{He}$-based neutron polarization and analysis techniques. We are developing and promoting the applications of neutron spin filters based on laser-polarized ${ }^{3} \mathrm{He}$. The technique has the unique capability to polarize a polychromatic neutron beam without introducing additional beam divergence. This is a very attractive feature for many materials science and fundamental physics applications. We are pursuing applications at the NCNR, the Intense Pulsed Neutron Source (IPNS) at Argonne National Laboratory, and the Los Alamos Neutron Science Center.

\section{ACCOMPLISHMENTS}

\section{Neutron Technology for Homeland Security}

We have participated in the development of three new ANSI Standards, N42.32, N42.34, and N42.35, which provide test and evaluation protocols for neutron detection in personal electronic radiation monitors ("pagers"), isotope identifier instruments, and portal monitors, respectively. We are managing the procurement, calibration, and dissemination of standard, low-intensity neutron sources to three national laboratories for testing of commercial instru- 
ments under these standards. We are also leading the development of new, additional ANSI standards on neutron survey meters and active interrogation devices for detection of fissile material, explosives, and chemical agents.

The challenge of calibrating low-intensity sources has motivated the development of a new source comparator with a reduced-volume manganese sulfate bath. For measurement of neutron radiation protection levels around active interrogation devices, we have procured and calibrated state-of-the-art commercial neutron spectrometry and absorbeddose measurement systems.

We are developing neutron spectrometers and epithermal/thermal neutron detectors with much higher sensitivity than current state-of-the-art systems. We are experimenting with muon anticoincidence techniques for reducing the cosmic-ray component of neutron background radiation. Finally, we have procured and calibrated a small, sealedtube source for generating $14 \mathrm{MeV}$ and $2.5 \mathrm{MeV}$ fast neutrons, for testing our new spectroscopy systems, and for developing radiation-protection dosimetry capabilities up to $14 \mathrm{MeV}$.

CONTACT: DR. DAVID M. GILLIAM

(301) 975-6206

david.gilliam@nist.gov

\section{New Imaging Station for}

\section{Fuel Cell Research}

The Neurron Imaging Facility started full-time operation in 2003 at Beam Tube 6 (BT-6) at the NCNR. The facility has an extremely high fluence rate of $1.8 \times 10^{7} \mathrm{~cm}^{-2} \mathrm{~s}^{-1}$ uniformly spread

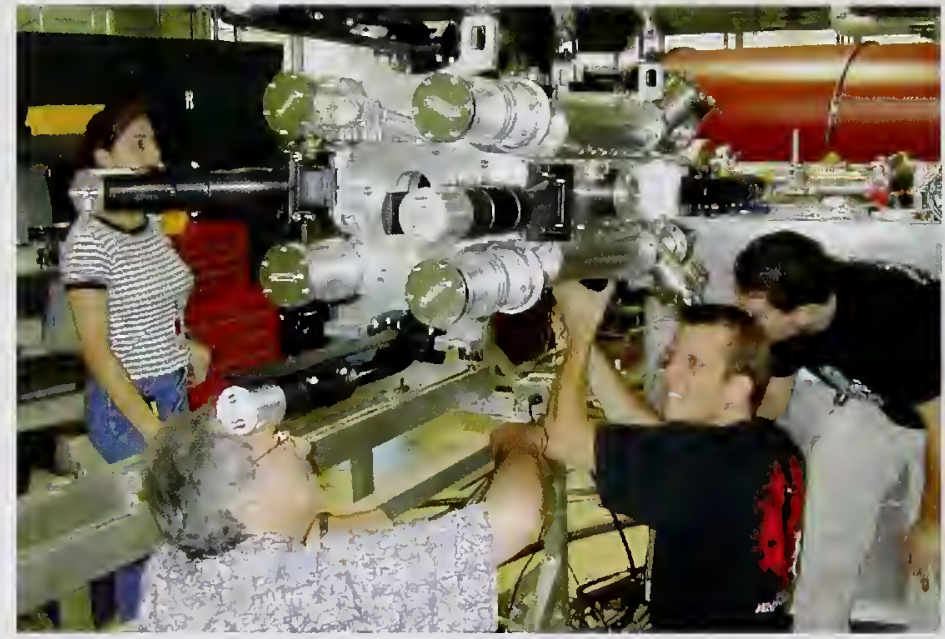

Figure 3. Pieter Mumm (second from right) and colleagues setting up a neutron decay detector for the time reversal asymmetry experiment (emiT) over a $26 \mathrm{~cm}$ diameter area, with an $L / d$ ratio of 300 , which is needed to achieve optimum geometric sharpness of the image. This is one of the most advanced neutron imaging facilities in the world and the best of its kind in the U.S.

We have pioneered nondestructive imaging techniques to map critically important hydrogen and water motion in operating fuel cells. This facility has been hosting experiments from industrial fuel cell developers, interested in looking at the distribution of water in operational fuel cell systems using standard fuel cell hardware. First-generation neutron experiments with a major industrial partner show exciting promise of robust/efficient fuel cell design, substantial reduction in fuel cell development time, and establishment of uniform characterization/performance standards.

This program has been awarded NIST competence research funding and, in 2003, DOE recognized this program as one of the top-ten programs relevant to the "FreedomCAR" program initiated by the President.

CONTACT: DR. DAVID L. JACOBSON

(301) 975-6207

david.jacobson@nist.gov
Neutron Measurements to Test "Standard Model"

The emiT experiment successfully completed its data acquisition on NG-6 in December 2003. (See Fig. 3.) The experiment is a collaboration among NIST, the University of Washington, the University of California (Berkeley), the University of Michigan, Hamilton College, and Tulane University.

The experiment searches for (or will set an improved upper bound on) the timereversal asymmetry term in neutron beta decay. It does so by measuring electronproton coincidence events from the decay of polarized neutrons. An asymmetry in coincidence pairs is formed as a function of the direction of the neutron spin. A measurement of a nonzero asymmetry would be an indication of time-reversal violation.

The measured electron-proton coincidence rate is a factor-of-ten higher than in the first run. In addition, the signalto-background ratio is two orders of magnitude higher. The analysis of the data is in progress, and we anticipate a result that is approximately a factor of four better than the world current limit.

Work has commenced at NG-6 on an effort to measure the radiative decay mode of the free neutron. The usual beta decay of the neutron into a proton, 
electron, and antineutrino is occasionally accompanied by the emission of a photon. Despite decades of detailed experimental studies of neutron beta decay, this branch of a fundamental weak decay has never been observed.

Our technique is based on measuring the triple coincidence of the proton and electron along with the rare photon. The experiment should not only definitively see radiative neutron beta decay for the first time, but also perform a $5 \%$ measurement of the photon spectrum.

CONTACT: DR. JEFFREY S. NICO

(301) 975-4663

jeffrey.nico@nist.gov

\section{Polarized ${ }^{3}$ He Neutron Spin}

\section{Filters for Fundamental and}

\section{Applied Research}

In collaboration with scientists in the NIST Materials Science and Engineering Laboratory and Indiana University, we are employing ${ }^{3} \mathrm{He}$-based polarization analysis for diffuse reflectometry at the new Advanced Neutron Diffractometer/Reflectometer at the NCNR, and the POSY-I reflectometer at IPNS.

Our current focus is on studies of patterned magnetic materials, which are relevant to the development of future magnetic storage media (Fig. 4). In addition, we are collaborating with researchers from Hamilton College, IPNS, and the Spallation Neutron Source in the application of a continuously operating ${ }^{3} \mathrm{He}$ polarizer for single-crystal diffractometry.

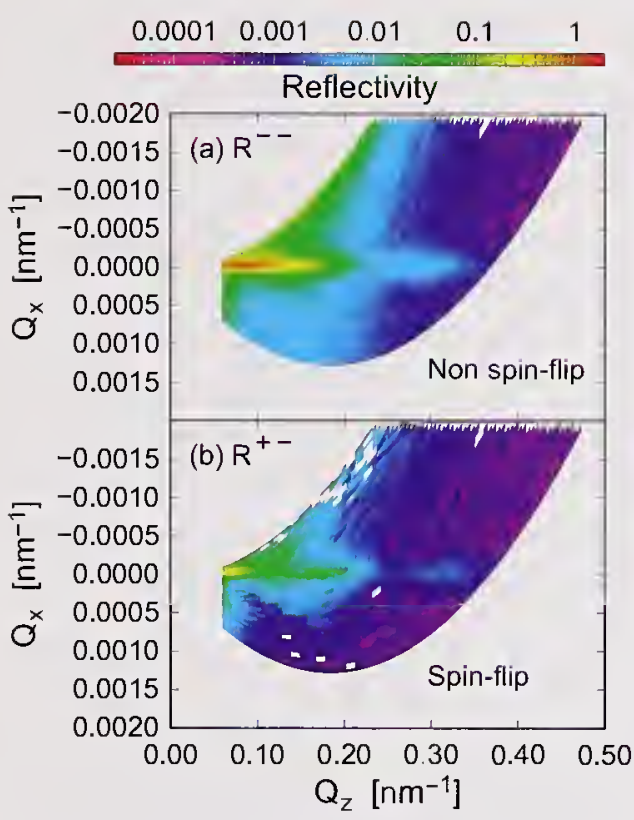

Figure 4. Polarization analysis of neutron reflection from a sample of cobalt "antidots,"

obtained with a ${ }^{3} \mathrm{He}$ analyzer and a positionsensitive detector. $Q_{z}$ and $Q_{x}$ are the $z$ and $x$ components of the wavevector transfer; spin-flip scattering from magnetic domains is evident in the off-specular data

In fundamental physics, we are contributing to the operation and further development of the ${ }^{3} \mathrm{He}$ polarizer for an experiment in weak interaction physics (the "npdy" experiment) at Los Alamos National Laboratory. In addition, we are developing ${ }^{3} \mathrm{He}$ cells for a planned measurement of the spin dependence of the ${ }^{3} \mathrm{He}$ scattering length on the NCNR neutron interferometer. In collaboration with the University of Utah, we have documented remarkable magnetic effects in the polarized ${ }^{3} \mathrm{He}$ storage cells, which are relevant to suppressing wall relaxation time.

CONTACT: DR. THOMAS R. GENTILE (301) $975-5431$

thomas.gentile@nist.gov he third strategic element is to develop dosimetric standards for $x$ rays, samma rays, and electrons based on the SI unit, the gray, for homeland security, medical, radiation processing, and radiation protection applications.

\section{Radiation Dosimetry Standards}

\section{INTENDED OUTCOME AND BACKGROUND}

The Radiation Interactions and Dosimetry Group promotes the accurate and meaningful measurements of dosimetric quantities pertaining to ionizing radiation: $\mathrm{x}$ rays, gamma rays, electrons, and energetic, positively charged particles. We maintain the national measurement standards for the Système International (SI) unit for radiation dosimetry, the gray.

NIST is a world leader in the measurement of high levels of absorbed dose, as required in the industrial radiation processing of materials (e.g., sterilization of single-use medical devices, food irradiation, and destruction of biological weapons). Accurate transfer dosimetry is increasingly done on the basis of alanine/EPR (Electron Paramagnetic Resonance) dosimetry, rather than radiochromic film dosimetry, which was originally developed at NIST and offered for many years as a calibration service. A new NIST system is near 
completion for on-demand, Internetbased $e$-calibrations for industry, based on alanine/EPR dosimetry.

Brachytherapy (treatment with sealed radioactive sources) has seen a tremendous increase in the use of low-energy, photon-emitting seeds to treat prostate cancer and in the use of beta-particle(and photon-) emitting sources to inhibit arterial restenosis (re-closing) following balloon angioplasty. In both cases, NIST has responded to the needs of the manufacturers, regulators, and clinical physicists. We develop new standards and measurement methods to calibrate the quantities needed to ensure accurate dosimetry for the wide variety of sources introduced, and we disseminate these standards through a network of secondary calibration laboratories.

Nearly 700,000 cancer patients per year are treated in the U.S. with radiation beams, mainly from high-energy electron accelerators (either directly with the electrons or by converting them to high-energy $\mathrm{x}$ rays). NIST maintains and disseminates the standards for air kerma (exposure) and for absorbed dose to water from ${ }^{60} \mathrm{Co}$ gamma-ray beams. These provide the basis for calibrating instruments used to measure the absorbed dose delivered in therapy beams.

Standards for diagnostic radiology are developed and maintained at NIST in terms of air kerma, for $\mathrm{x}$-ray beams from $10 \mathrm{kV}$ to $300 \mathrm{kV}$ (x-ray source accelerating potential in kilovolts). These are disseminated to manufacturers and the medical physics community in North
America through a network of secondary calibration laboratories. NIST maintains some 85 beam qualities for conventional, $\mathrm{W}$-anode, $\mathrm{x}$-ray beams, and 17 beam qualities for mammographic, Mo- and Rh-anode, $\mathrm{x}$-ray beams.

The radiation transport and Monte Carlo methods pioneered and developed at NIST to calculate the penetration of electrons and photons in matter are used in most of the major codes today. Monte Carlo simulation is increasingly applied to problems in radiation metrology, protection, therapy, and processing as an accurate tool for designing and optimizing radiation systems and for providing important insight into processes inaccessible to measurement.

\section{ACCOMPLISHMENTS}

\section{Assistance to U.S. Postal Service in Decontamination of Mail}

After anthrax-laced mail was delivered to media and government offices, resulting in five deaths, numerous illnesses, and enormous disruption and economic loss, we responded rapidly to identify industrial irradiation of the mail as an effective and readily available process to kill anthrax spores. Leading a task force established by the White House Office of Science and Technology Policy, we worked with the Armed Forces Radiobiology Research Institute, the U.S. Postal Service (USPS), and industrial irradiation facilities to provide critical dosimetry measurements and to validate the process.

Based on an extensive program of Monte Carlo radiation transport calculations and accurate dosimetry measure- ments in a variety of mail configurations, NIST provided advice for optimizing the process parameters and developing a national strategy to effectively handle the highly variable mail and parcel stream.

This collaboration continues, expanded to include qualitative measurements of radiolytic products produced in the mail during the irradiation, quantitative measurements of the effects on the archival properties of paper due to irradiation, and the design of a dedicated USPS mail-irradiation facility.

CONTACT: MR. STEPHEN M. SELTZER (301) 975-5552

stephen.seltzer@nist.̧ov

\section{High-Energy Computed Tomography Facility}

The $7 \mathrm{MeV}$ to $32 \mathrm{MeV}$ Saggataire electron linac in the NIST Medical Industrial Radiation Facility (MIRF) offers unique possibilities as an $\mathrm{x}$-ray source for a high-energy computed tomography (HECT) facility. A beamline and camera are under development at MIRF to study the $\mathrm{x}$-ray inspection of cargo containers, trucks, and other large objects.

We are also carrying out theoretical and experimental investigations into neutron production in high-energy $\mathrm{x}$-ray beams. It might prove possible to also use photoneutrons, produced at high photon energies, as an active probe to interrogate containers and screen for explosives and other terrorist materials.

CONTACT: MR. JULIAN H. SPARROW (301) 975-5578

julian.sparrow@nist.gov 


\section{Radiation Sources for Medical and Industrial Applications}

In addition to a dozen gamma-ray $\left({ }^{60} \mathrm{Co}\right.$ and $\left.{ }^{137} \mathrm{Cs}\right)$ sources and five $\mathrm{x}$-ray ranges, we maintain MIRF, a $4 \mathrm{MeV}$ electron Van de Graff, a $500 \mathrm{keV}$ electrostatic electron accelerator, and a $1 \mathrm{MeV}$ to $8 \mathrm{MeV}$ pulsed electron linac. These are used in a variety of radiation applications such as material modification, radiation-hardness testing, electron- and bremsstrahlung-beam dosimetry, and HECT development.

New accelerator facilities, incorporating machines currently used in medical and industrial applications, are bringing our programs to the forefront of research and development. Installation of a $6 \mathrm{MeV}$ to $20 \mathrm{MeV}$ electron-beam (6 MV and $18 \mathrm{MV}$ bremsstrahlungbeam) Varian Clinac $2100 \mathrm{C}$ to support the development of direct, therapylevel dosimetry calibrations has been completed. (See Fig. 5.)

Planning is underway for a facility based on two Titan $10 \mathrm{MeV}, 17 \mathrm{~kW}$ electron linacs, donated by the U.S. Postal

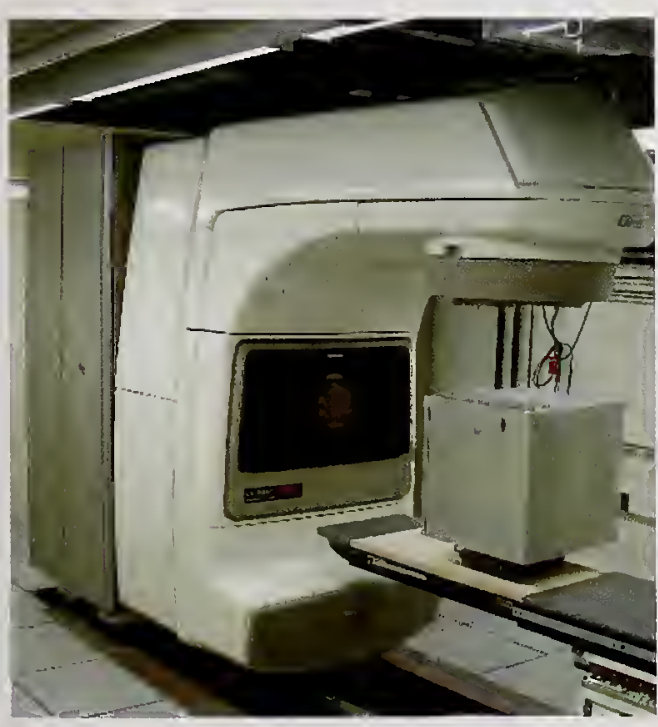

Figure 5. NIST medical-therapy accelerator facility
Service, to support the standards and calibrations program for homeland security applications, general industrial radiation processing, and the study of radiation effects in materials.

CONTACT: DR. FRED B. BATEMAN

(301) 975-5580

fred.bateman@nist.gov

\section{Calibration of Low-Energy Photon Brachytherapy \\ Sources}

Small radioactive "seed" sources used in prostate brachytherapy, containing the radionuclide ${ }^{103} \mathrm{Pd},{ }^{125} \mathrm{I}$, or ${ }^{131} \mathrm{Cs}$, are calibrated in terms of air-kerma strength using the NIST Wide-Angle Free-Air Chamber (WAFAC). The WAFAC is an automated, free-air ionization chamber with a variable volume, allowing corrections to be made for passage of the beam through non-air-equivalent electrodes. Over 500 seeds of 32 different designs, from 18 manufacturers, have been calibrated using the WAFAC since 1999. (See Fig. 6.)

On-site characterization at seed manufacturing plants for quality control, as well as at therapy clinics for treatment planning, relies on well-ionization chamber measurements. Following the primary standard measurement of airkerma strength, the responses of several well-ionization chambers to the various seed sources are determined. The ratio of air-kerma strength to well-chamber response yields a calibration coefficient for the well-ionization chamber for a given seed type. Such calibration coefficients enable well-ionization chambers to be employed at therapy clinics for verification of seed air-kerma strength, which is used to calculate dose rates to ensure effective treatment planning.

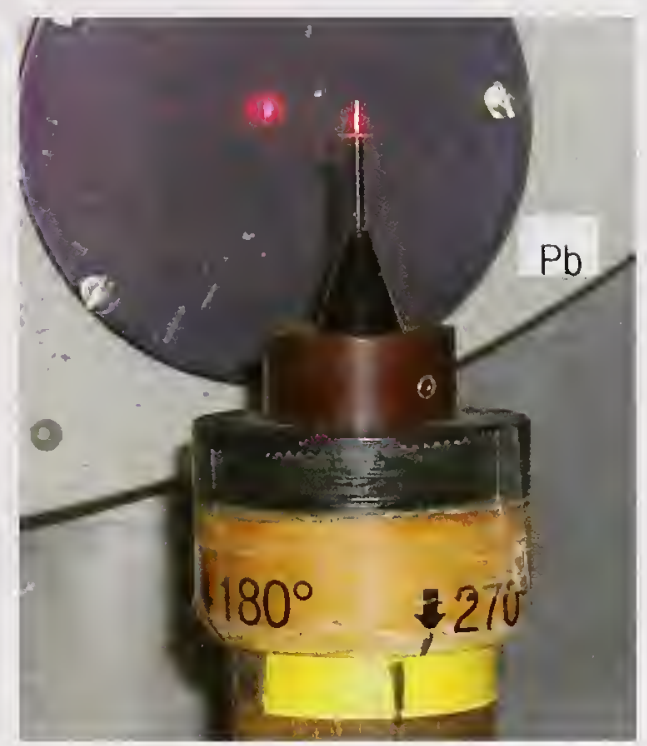

Figure 6. Close-up view of a seed (illuminated by a laser), mounted vertically on a rotating post, ready for calibration on the WAFAC calibration range.

To verify that seeds of a given design calibrated at NIST are representative of the majority of those calibrated in the past, several additional tests have been implemented. The distribution of radioactive material within a seed is mapped using radiochromic film contact exposures. The in-air anisotropy of seeds is studied by taking WAFAC and $\mathrm{x}$-ray spectrometry measurements at discrete rotation angles about the long axis and the axis perpendicular to the midpoint of the long axis of the seed, respectively. The "air-anisotropy ratio," calculated from the results of angular $x$-ray measurements, has proven to be a useful parameter for explaining differences in well-chamber response observed for different seed models having the same emergent spectrum on their transverse axis.

CONTACT: DR. MICHAEL G. MITCH (301) 975-5491

michael.mitch@nist.gov 


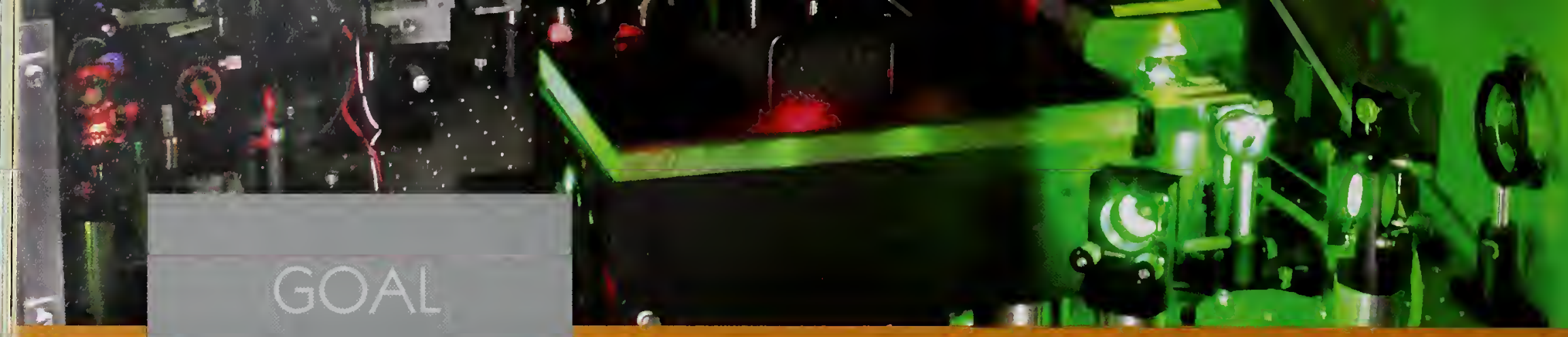

TO PROVIDE THE

FOUNDATION OF FREQUENCY

MEASUREMENTS AND CIVIL

TIMEKEEPING FOR

OUR NATION

\section{TIME AND FREQUENCY DIVISION}

The strategy of the Time and Frequency Division is to advance measurement science and to provide time and frequency standards and measurement services to commerce, industry, and the public. he first strategic focus

is to develop the standards

that serve as reference for

time and frequency services

and to research advanced

measurement systems.

\section{Time and Frequency}

\section{Standards}

\section{INTENDED OUTCOME AND}

BACKGROUND

The Time and Frequency Division maintains standards with the accuracy, continuity, and stability essential for supporting U.S. commerce and scientific research; provides an official source of time for U.S. civilian applications; and supports coordination of international time and frequency standards, including realization of the SI second.

NIST time and frequency standards are based on the NIST time scale and the
NIST primary frequency standard, NIST-F1. The time scale is an ensemble of five (soon, six) hydrogen masers and four cesium-beam clocks. The stability of the time scale is approximately $0.2 \mathrm{fs} / \mathrm{s}$ for thirty days of averaging, with a long-term frequency drift of less than $3(\mathrm{fHz} / \mathrm{Hz}) / y e a r$. The frequency of the time scale is calibrated by periodic comparisons to the NIST-F1 laser-cooled cesium primary frequency standard (9.2 GHz microwave frequency), with a fractional frequency uncertainty $\Delta f f f$ approaching $6 \times 10^{-16}(0.6 \mathrm{fHz} / \mathrm{Hz}$, as of June 2004).

The NIST time scale is the basis of NIST's realization of Coordinated Universal Time (UTC), the international time scale. NIST is one of about 60 timing laboratories across the world continuously contributing to the realization of UTC. Through improvements to the NIST time scale, NIST's realization of UTC rarely differs from the international average by more than 10 nanoseconds. In addition, NIST is one of only four laboratories worldwide (as of late 2004) operating the highest accuracy primary frequency standards to determine the frequency (rate) of UTC.
The extraordinarily stable NIST time scale, coupled with world-leading performance of the NIST primary frequency standard (as of June 2004), provides U.S. industry and science with a unique resource for the most demanding applications of accurate time and frequency. However, commercial and scientific needs for even more accurate and stable time and frequency standards drive a vigorous NIST research program to improve microwave frequency standards and to develop new, optical frequency standards.

Since the first atomic clock was invented at the National Bureau of Standards (NIST's predecessor) in 1949, the performance of primary frequency standards has consistently improved by about a factor of ten each decadedriven by, and enabling, advances such as telecommunications synchronization and the Global Positioning System (GPS). NIST research on microwave and optical frequency standards strives to at least maintain this rate of performance improvement. 
ACCOMPLISHMENTS

Improved Performance of the NIST-F1 Primary

\section{Frequency Standard}

The NIST-F1 laser-cooled, cesium fountain primary frequency standard

(Fig. 1) is the U.S. national standard for frequency and the realization of the SI second. Since the first formal report of NIST-F1 frequency to the International Bureau of Weights and Measures (BIPM) in 1999, the NIST-F1 uncertainty has been reduced by about a factor of three.

The NIST-F1 frequency evaluation reported to BIPM in June 2004 included an "in-house" fractional frequency uncertainty of approximately $6 \times 10^{-16}$ $(0.6 \mathrm{fHz} / \mathrm{Hz})$, increasing to about $9 \times 10^{-16}(0.9 \mathrm{fHz} / \mathrm{Hz})$ as received at BIPM due to uncertainties in the satellite-transfer process. Both of these results were, at the time, the best ever reported to BIPM - by a significant margin.

Ongoing NIST-F1 changes continue to improve performance. New laser systems, for generating the optical molasses and repumping, maintain frequency lock for weeks without intervention. Since NIST-F1 data must be averaged for about a month to achieve ultimate uncertainties, this improvement has substantially reduced downtime. The higher power molasses-laser system also permits reshaping of the atom cloud to reduce atom density in the vertical (launch) direction, decreasing the spin-exchange shift without loss in detected atom number. Systems that ensure that mechanical shutters fully block the lasers

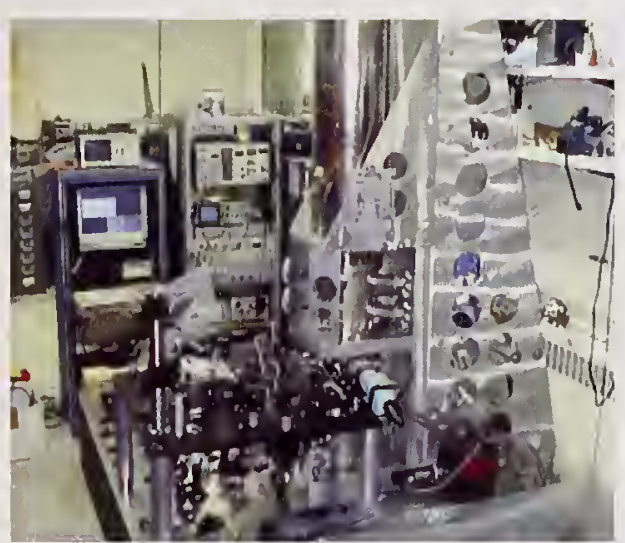

Figure 1. The NIST-F1 cesium fountain primary frequency standard, the Nation's standard for frequency and the SI second.

have reduced the light-shift uncertainty by $50 \%$. Other improved systems include 9.2 GHz microwave synthesis, vacuum, temperature, computer automation, and data transfer to the time scale.

We are carefully exploring the role of deadtime in precision frequency measurement and satellite transfer-that is, time when the NIST maser ensemble continues to operate, and potentially drift, while the primary frequency reference is not operating. In addition to purposely building deadtime into formal frequency evaluations, the Division is collaborating with the NIST Statistical Engineering Division to formally model deadtime effects.

\section{CONTACT: DR. STEVEN JEFFERTS}

(303) 497-7377

jefferts@boulder.nist.gov

\section{Second-Generation Cesium Fountain Primary Frequency Standard}

While continuing to optimize NIST-F1, the Division is actively developing the next-generation primary frequency standard, NIST-F2. The largest nonstatistical contributions to NIST-F1 frequency uncertainty are the spin-exchange shift, due to cold atom collisions, and the blackbody shift (AC Stark shift from thermal radiation near $300 \mathrm{~K}$ ). NIST-F2 is intended to substantially reduce those uncertainties through two means.

First, NIST-F2 will use a multi-toss, multiple-velocity system, in which about ten low-density atom balls will be launched to different heights in rapid succession, all coalescing in the detection zone without having crossed paths in the Ramsey interrogation region. This approach will minimize spinexchange shifts while still providing sufficient atom numbers at detection for good stability.

The general principles of this approach have been successfully demonstrated in NIST-F1. To make the system practical, NIST-F2 will use a low-velocity intense source (LVIS) of atoms for the required rapid cooling, capture, and launch of many atom balls in succession. Early results demonstrate molasses atom fill rates of about $4 \times 10^{9} \mathrm{~s}^{-1}$ In addition, the fountain team has demonstrated cooling to approximately $1 \mu \mathrm{K}$ using the required $(1,1,1)$ NIST-F2 fountain geometry. Both these results are commensurate with NIST-F2 performance requirements.

The second major improvement in NIST-F2 will be to cool the drift tube and interrogation regions to cryogenic temperatures, vastly reducing the blackbody shift. Use of different cryogens, and/or pumping on the cryogens, will also enable accurate measurement of the blackbody shift, the value of which has been the subject of intense debate. 
The ultimate goal for NIST-F2 is to approach an "in-house" fractional frequency uncertainty of $1 \times 10^{-16}$ in the next few years.

CONTACT: DR. ELIZABETH DONLEY (303) 497-5173

edonley@boulder.nist.gov

\section{Research on Optical Frequency Standards}

While the ultimate accuracy limit of cesium microwave standards operating near $10^{10} \mathrm{~Hz}$ is not yet clear, optical frequency standards operating on the order of $10^{15} \mathrm{~Hz}$ have the potential for substantially greater stability and accuracy. Optical frequency standards also have a potential for dissemination through optical fiber, which may be advantageous in many applications.

The Division conducts a vigorous research program on prospective optical frequency standards, simultaneously pursuing several different approaches. These include cold, trapped single ions; cold, neutral atom clouds; and "logic clocks," using techniques of quantum computing. A crucial part of optical clock research is optical frequency synthesis using femtosecond laser frequency combs, described in a later section.

\section{Mercury-Ion Optical}

\section{Frequency Standard}

A frequency standard based on optical transitions $(282 \mathrm{~nm}, 1064.7 \mathrm{THz})$ in a single, laser-cooled, trapped mercury ion has potential for better accuracy than cesium fountain standards by a factor of 100 or more. With a $Q$ factor $>10^{14}$, and a transition that is relatively insensitive to environmental factors, the potential fractional frequency uncertainty $\Delta f l f$ for a mercury ion standard is as small as $10^{-18}$.

The reproducibility of the mercury ion standard relative to the NIST-F 1 primary frequency standard was studied over a two-year period. Measurements were referenced to NIST-F1 through a hydrogen maser, using a femtosecond laser frequency comb to compare optical and microwave frequencies. The mercury-ion optical frequency standard has better short-term stability than NISTF1, so the stability of the maser was key to the intercomparison.

The experiment set a limit on the fractional frequency variation of the NIST-F1 cesium microwave transition, referenced to the mercury ion optical transition, as no greater than $\pm 7 \times 10^{-15}$ per year. From the point of view of the cesium transition being defined as the international frequency standard, this means the mercury ion clock frequency is stable to this level.

This experiment also set a limit on possible variations of fundamental constants related to the fine-structure constant $\alpha$, in particular the possible temporal variation of the quantity $g_{C_{s}}\left(m_{\mathrm{e}} / m_{\mathrm{p}}\right) \alpha^{6}$. Assuming any variation is due to $\alpha$ only, this result sets an upper bound for the fractional change in the fine-structure constant as no greater than $1.2 \times 10^{-15}$ per year. It is a tighter bound by about a factor of 30 than astronomical observations suggesting variations in the finestructure constant over periods comparable to the age of the universe.

CONTACT: DR. JAMES C. BERGQUIST

(303) 497-5459

berky@boulder.nist.gov

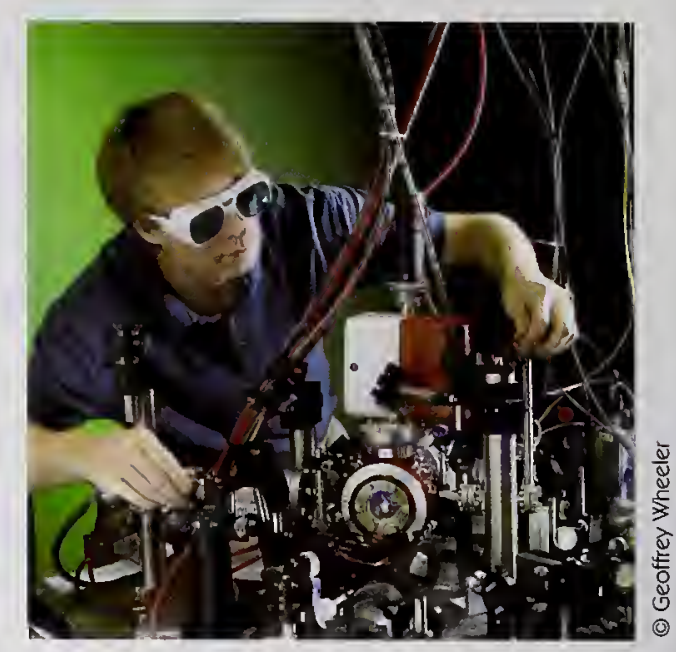

Figure 2. The calcium-atom optical frequency standard, being adjusted by Chris Oates.

\section{Calcium-Atom Optical}

\section{Frequency Standard}

The calcium optical clock transition at about $657 \mathrm{~nm}(456 \mathrm{THz})$ has several advantages. It is spectrally sharp ( $470 \mathrm{~Hz}$ natural linewidth), can be detected with excellent signal-to-noise using shelving-detection techniques, and is largely insensitive to perturbations from external electric and magnetic fields and from cold collisions.

The frequency of the calcium optical clock transition has been measured to a fractional uncertainty approaching $1 \times 10^{-14}$, making it one of the bestknown optical frequencies. The $423 \mathrm{~nm}$ resonance line permits rapid first-stage laser cooling to the Doppler limit of about $2 \mathrm{mK}$, and we have demonstrated three-dimensional, quenched narrowline second- and third-stage laser cooling to about $10 \mu \mathrm{K}$. Experiments to slow calcium atoms further using pulsed, one-dimensional, quenched narrow-line laser cooling have reached temperatures as low as $300 \mathrm{nK}$. (See Fig. 2.)

A calcium optical frequency standard should ultimately demonstrate fractional stability approaching $2 \times 10^{-16}$ with one second of averaging. Performance comparable to cesium fountain microwave standards could be attained with averaging over seconds, rather than weeks. 
It is likely that ion and neutral atom optical standards will each display ultimate strengths and limitations. They may find uses in different applications in much the same way that different microwave standards are optimal for different applications—-such as cesium standards for absolute accuracy and hydrogen masers for greatest stability.

CONTACT: DR. CHRIS OATES

(303) 497-7654

oates@boulder.nist.gov

he second strategic focus is to develop and operate the frequency and time services essential for synchronizing important industrial/commercial operations and supporting trade and commerce.

\section{Time and Frequency Services}

\section{INTENDED OUTCOME AND}

\section{BACKGROUND}

The Division provides continuous, reliable, time and frequency signals in a wide variety of formats and accuracies to meet diverse needs of U.S. industry, trade, science, and the general public. NIST time and frequency information is distributed over the Internet, by radio broadcasts, over telephone lines, and through satellites to serve customers in finance, telecommunications, science, transportation, radio/TV broadcasting, and other businesses-and as a reliable and convenient source of official U.S. time for the general public.

NIST radio stations WWV in Ft. Collins, Colorado and WWVH in Kauai, Hawaii broadcast shortwave radio signals containing a rich variety of time and frequency information, in the form of verbal announcements, tones, and digital time codes. NIST radio station WWVB in Ft. Collins, Colorado broadcasts a low-frequency $(60 \mathrm{kHz})$ digital time code that automatically sets consumer timepieces to official U.S. time and date, automatically correcting for daylight saving time, leap years, and leap seconds.

NIST's most heavily used service is the Internet Time Service (ITS), automatically setting clocks in computers and nerworked devices to NIST time. The Division also provides the modem-based Automated Computer Time Service (ACTS) to set computer and network device time. Many ACTS customers need the security of a direct connection to NIST to ensure that the time is legally traceable to NIST and is auditable. For example, the National Association of Securities Dealers (NASD) requires its 600,000 members to time-stamp many billions of dollars of electronic transactions each business day against NIST time.

The Frequency Measurement and Analysis Service (FMAS) serves industrial and research customers who need tight traceability to NIST frequency standards. FMAS customers receive continuous, real-time NIST traceability through a highly automated system remotely monitored by NIST, receiving NIST frequency standards by comparison to GPS broadcasts. The most demanding customer needs are met through the Global Time Service, which uses GPS common-view time transfer to deliver to the user's site a timing reference very close in accuracy to the NIST time scale.

To enhance U.S. expertise in this field, the Division offers a variety of training courses. A $31 / 2$ day metrology seminar is offered annually, a new 3 day seminar has been initiated for entry-level time and frequency metrologists, and we cosponsor an annual workshop on synchronization in telecommunications systems. NIST staff members also teach courses at conferences.

\section{ACCOMPLISHMENTS}

\section{NIST Internet Time Service (ITS)}

Fourteen ITS servers at eleven locations across the Nation respond to about $1 \frac{1}{2}$ billion requests per day (as of summer 2004) for setting computer and network clocks to NIST time, automatically. (See Fig. 3.) The Division website provides free client software, complete

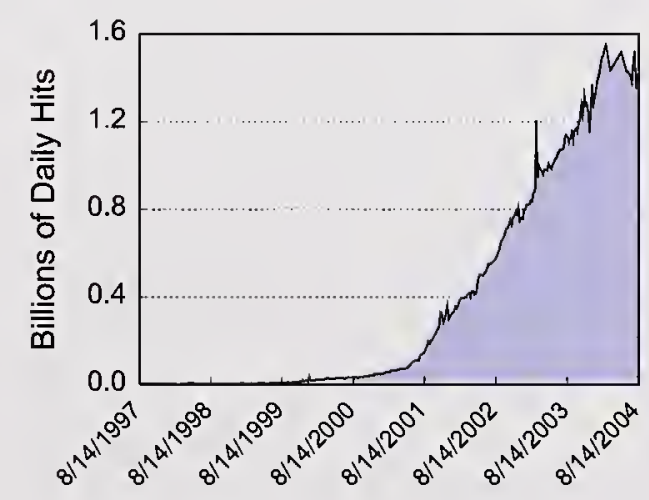

Figure 3. Growth in use of the NIST Internet Time Service. 
source code, and complete instructions (about 100,000 downloads per month). Automatic synchronization to ITS is now a capability of the most popular computer operating systems, including Windows XP, Mac OS, and many commercial versions of Linux.

The Division continually updates the servers, software, and network infrastructure, working with the NIST networking group, to ensure continued provision of ITS in light of increasing demands.

A number of companies have partnered with NIST to add traceability and auditability to ITS for timestamping electronic documents and financial transactions. These applications are in rather limited use now, but are growing rapidly. It is likely that they will become part of the national information infrastructure. For example, the U.S. Postal Service Electronic Postmark program places a secure, auditable timestamp on electronic documents referenced to NIST time, and stores these documents on secure servers for parties in a transaction to examine and exchange as legal documents.

CONTACT: DR. JUDAH LEVINE

(303) 497-3903

jlevine@boulder.nist.gov

\section{NIST Radio Stations WWV, WWVH, and WWVB}

The Division continues to upgrade the radio stations as part of a long-term modernization plan. Progress includes continuing improvements to WWV and WWVB transmitters and broadcast systems, including systems to ensure automatic recovery from power failures or

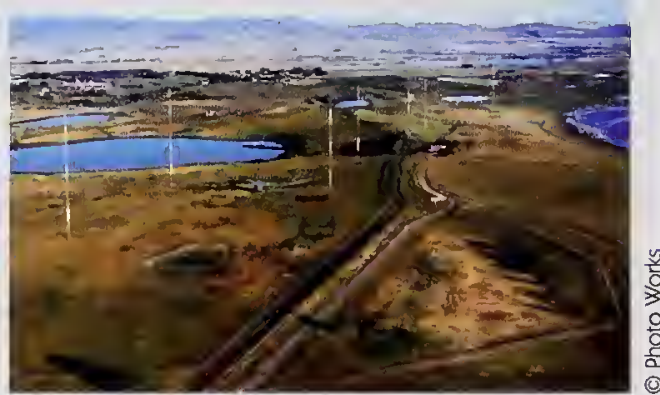

Figure 4. An aerial view of the WWNB antenna systems.

loss of primary transmitters when station staff are not present; continuing replacement of WWVH metal antenna towers, subject to corrosion from sea salt, with fiberglass towers hinged to permit bending the tower to the ground for easy and safe maintenance; and upgrading insulators in the guy wires supporting the eight 122 meter (400 foot) towers for the WWVB antennas (Fig. 4).

More than 50 manufacturers produce WWVB-controlled timepieces, with several million new units sold each year. With growing sales, both manufacturers and consumers want to know that WWVB signal strength is sufficiently strong across the Nation to ensure good reception. We designed a monitor system to measure received power from WWVB broadcasts, and several of these systems were constructed on contract. We plan to place them at strategic locations around the U.S. Through an Internet connection, each will continuously report back the WWVB signal strength to the Division. The results will be displayed on a publicly available website, along with archives of all signal strength records.

We have been actively consulting with WWVB-controlled timepiece manufacturers to improve their product performance. The monitoring data will help manufacturers set performance specifications for real-world signal strengths. Consumers can also consult the website to determine if problems with their timepieces result from inadequate WWVB signals or, as is almost always the case, from problems with the timepiece itself.

The Division is publishing a

"Recommended Practices for Manufacturers and Consumers of WWVB Radio Controlled Clocks" guide, developed in consultation with manufacturers and enthusiast user groups, which suggests practices to ensure optimal timepiece performance.

CONTACT: MR. JOHN P. LOWE

(303) 497-5453

lowe@boulder.nist.̧ov

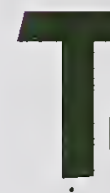

he third strategic focus is to develop new measurement systems and methods in support of emerging technologies.

\section{New Measurement Systems and Methods}

\section{INTENDED OUTCOME AND \\ BACKGROUND}

In addition to meeting current customer needs, the Division prepares for the future of time and frequency measurements and calibrations. Through interactions and discussions with constituents, we identify important emerging requirements and technologies. We 
strive to apply our expertise and creativity to those applications with the potential for greatest impact on U.S. industry, science, and the general public.

Synthesis and measurement of optical frequencies is crucial to the future of Division programs, and time and frequency metrology in general. Division expertise in developing and applying frequency combs based on femtosecond lasers has led to measurement of frequencies with relative uncertainties approaching $10^{-19}(0.1 \mathrm{aHz} / \mathrm{Hz})$, orders of magnitude better than previously possible, and to direct comparison of microwave and optical frequency standards, bridging five decades in frequency. We are working on techniques for amplification, noise reduction, and applications across different frequency ranges, such as the important nearinfrared telecommunications range.

A second key thrust is development of new tools to better measure close-to-carrier noise in oscillators and other electronic components. Such measurements are crucial to development of new oscillators, microwave and optical, used in advanced radars, telecommunications, high-speed digital circuits, and many other applications. Much of this work is conducted with significant support from DARPA, involving NIST, industry, and research organizations.

A third major program is the development of ultra-miniature atomic frequency standards, to dramatically improve the performance of small electronic devices such as GPS receivers and wireless communications devices. Such chipscale atomic clocks need not be as accurate or stable as large laboratory standards, but they will bring atomically

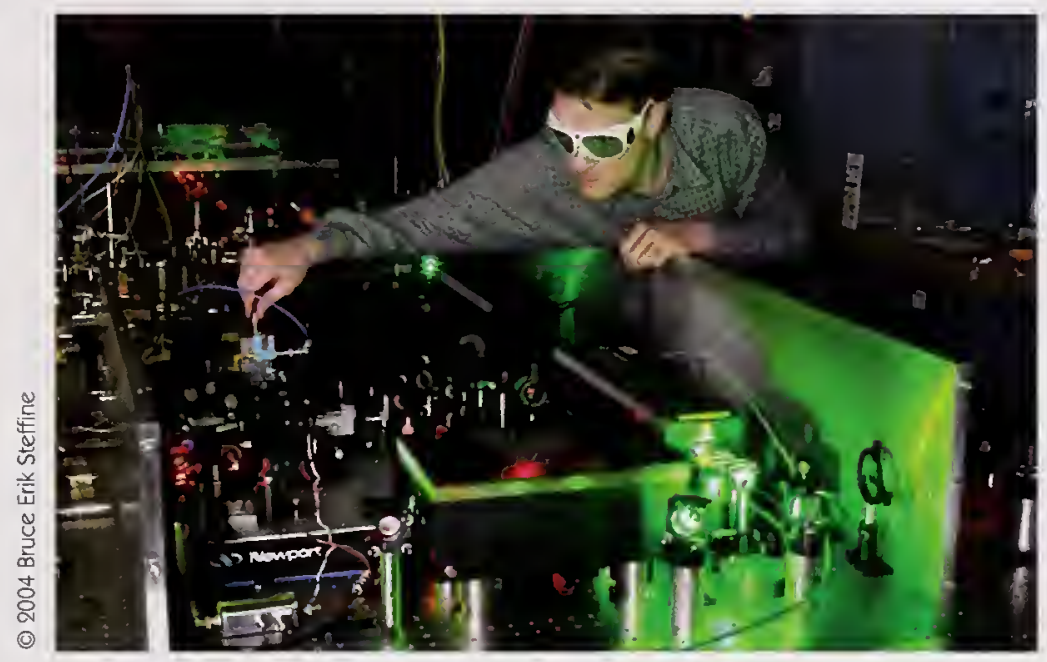

Figure 5

Scott Diddams with a femtosecond laser-based optical frequency synthesizer system. precise timekeeping and frequency control to small, battery-powered electronic devices.

DARPA and other funding agencies support the Division's participation in government-industry-university collaborations, recognizing that our core expertise in research and metrology accelerates the development of commercial and military products and services with strategic national economic and security impacts. This support is one important way the Division ensures that programs are well aligned with high-priority industrial and national needs.

\section{ACCOMPLISHMENTS}

\section{Improvements in Frequency combs}

A key application of frequency combs based on femtosecond lasers is to generate an arbitrary optical or microwave frequency output given an optical frequency reference input. This remarkable capability is crucial to the development and dissemination of useful optical frequency standards. As mentioned, the Division and other laboratories have used optical frequency combs to directly compare the cesium fountain microwave frequency $(9.2 \mathrm{GHz})$ with optical fre- quencies from the calcium atom standard $(456 \mathrm{THz})$ and the mercury ion standard (doubled $532 \mathrm{THz}$ ).

The Division has been continually improving the performance and versarility of frequency combs by exploring new ways to broaden the femtosecond laser output without use of microstructured optical fibers, which are susceptible to damage. (See Fig. 5.) The Division also collaborates with the NIST Electronics and Electrical Engineering Laboratory to develop Cr:fosterite femtosecond lasers, operating in the nearinfrared from about $1.0 \mu \mathrm{m}$ to $2.2 \mu \mathrm{m}$, including the important $1.4 \mu \mathrm{m}$ to $1.6 \mu \mathrm{m}$ optical telecommunications band.

An indication of progress is the recent Division-led frequency intercomparison of four different femtosecond laser frequency synthesizers, from three laboratories, using two fundamentally different comb-generation techniques, broadband operation and nonlinear microstructure fiber. Three frequency synthesizers—one each from the International Bureau of Weights and Measures, East China Normal University, and the Division-were compared to a second Division one, 
referenced to a $456 \mathrm{THz}$ optical frequency standard. Comparisons were conducted for a total of six days spread across several months.

The frequency differences, determined by optical heterodyne techniques, were measured to a relative uncertainty of $1.4 \times 10^{-19}$, with the uncertainty arising primarily from mechanical and thermal effects and limits on integration time. The results suggest optical frequency combs can be reliably used for frequency comparisons and synthesis to at least a fractional uncertainty of $10^{-19}$, and likely better when technical noise (mechanical and thermal fluctuations) are better controlled and longer integration times are used.

CONTACT: DR. SCOTT A. DIDDAMS (303) 497-7459

sdiddams@boulder.nist.gov

\section{Chip-Scale Atomic Clock}

Division research on a miniature, alloptical atomic clock, based on coherent population trapping, stimulated DARPA interest in developing a chip-scale atomic clock (CSAC). Program goals are to bring atomically precise timing and frequency control to applications in portable electronic devices, such as enhanced GPS receivers and better performing wireless communications devices for improved communications security. The overall goals of the DARPA program are to develop a CSAC of $1 \mathrm{~cm}^{3}$ total volume, consuming no more than $30 \mathrm{~mW}$ of power with a fractional frequency stability of about $1 \times 10^{-11}$ over one hour.

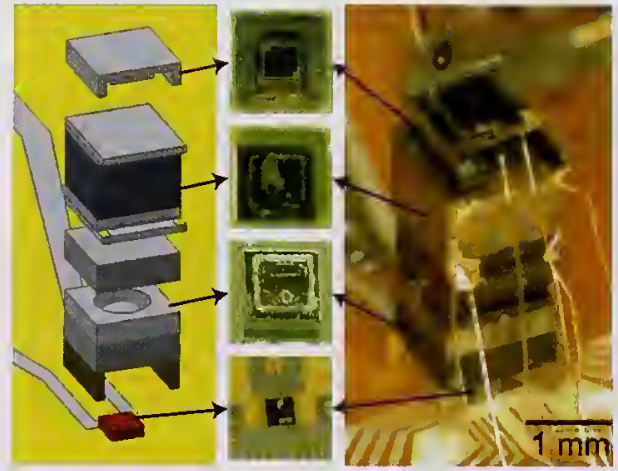

Figure 6. Photomicrograph of the physics package of a NIST chip-scale atomic clock, with a schematic diagram.

NIST is part of a DARPA-funded CSAC consortium of eight companies and universities. The Division is charged with providing the fundamental research and metrology, with the expectation that companies will develop the commercial products.

The Division has recently demonstrated a prototype CSAC with a physics package smaller than $0.01 \mathrm{~cm}^{3}$, a fractional frequency instability of about $2.5 \times 10^{-11}$ at 250 seconds integration, and a power consumption of about $75 \mathrm{~mW}$ (Fig. 6). These parameters are believed to be best in the world, and are approaching the stringent DARPA goals. The Division understands what modifications are needed to significantly improve the frequency stability and reduce the power consumption, and is optimistic that the DARPA goals can be met relatively soon.

The Division has collaborated with the NIST Electronics and Electrical Engineering Laboratory to use standard MEMS fabrication techniques in making the CSAC physics package, suggesting that CSACs based on the Division model could be mass-produced at relatively low cost, using wafer-level assembly techniques. Such a process would enable the extremely broad application of CSACs in portable electronic devices, for many applications.
The Division is also exploring the possibility of using CSAC technology to make tiny magnetometers approaching the sensitivity of SQUIDs, without the need for cryogens, and for other applications.

CONTACT: DR. JOHN KITCHING

(303) 497-4083

kitching@boulder.nist.gov

he fourth strategic focus is to develop quantum-logic components and quantum information systems based on trapped ions, in support of new atomic frequency standards and a national program aimed at advancing computation and communication.

\section{Quantum Information Processing Using Trapped lons}

\section{INTENDED OUTCOME AND \\ BACKGROUND}

We conduct research on the development and properties of prototype quantum-logic devices consisting of small numbers of electromagnetically trapped and laser-cooled ions serving as quantum bits (qubits). This research comprises quantum computing, quantum measurement (including noise reduction in frequency standards), and develop- 
ment of new classes of quantum-logicbased frequency standards.

This project arose as part of a long-term research program on ion-based frequency standards. In particular, the goal of reducing fundamental quantum projection noise suggested the possibility of using similar approaches for quantum computing and quantum metrology. Division researchers soon became leaders in quantum computing research, and NIST-wide programs in quantum computing and quantum communications rapidly developed and demonstrated significant success.

Our focus on quantum computing meets two primary needs. First, quantum computing research is a national priority to ensure economic and physical security, with substantial investment by both defense and civilian funding agencies. Our unique expertise in quantum state engineering has made the trapped-ion quantum computing program a world-leading effort.

But the Division work on quantum state engineering also directly serves our time and frequency metrology mission. For example, we recently demonstrated Heisenberg-limited spectroscopy with three entangled ions, in a scheme that could be scaled to an arbitrary number of ions or atoms. In principle, this could dramatically reduce the averaging time required for a frequency standard to reach its statistical uncertainty limit, substantially improving the performance, and broadening the applications, of atomic clocks.
ACCOMPLISHMENTS

\section{Progress in Quantum State Manipulation for Quantum Computing and Quantum}

\section{Measurement}

The Division's quantum computing and quantum measurement program continues to make strong progress.

Division researchers demonstrated the ability to sympathetically laser-cool ions of different species in a trap. In one experiment, a two-ion crystal consisting of $\mathrm{a}^{9} \mathrm{Be}^{+}$ion and $\mathrm{a}^{24} \mathrm{Mg}^{+}$ion was cooled by laser irradiation on only one ion. The laser-cooled "refrigerant" ion sympathetically cooled the other ion through the Coulomb interaction, but because transitions in the two ions were separated by some $30 \mathrm{~nm}$ in wavelength, irradiation of one ion had no direct effect on the other.

This property enables an entangled pair of ions of different species to be laser cooled without perturbing the qubit information. Such a process could be very useful in a complex, multi-ion quantum computing architecture, where motional heating from quantum state manipulations must be removed without perturbing qubit states.

Sympathetic cooling also broadens the range of ions that can be used as potential frequency standards. For example, ${ }^{27} \mathrm{Al}^{+}$has potentially good clock transitions but no easily accessible laser cooling transitions. The Division is investigating the potential of $\mathrm{a}^{27} \mathrm{Al}^{+}$frequency standard using ${ }^{9} \mathrm{Be}^{+}$sympathetic cooling and entangled state spectroscopy.

Division researchers have conducted many experiments demonstrating the effectiveness of various logic gates and demonstrating quantum computing architectures that are, in principle, completely scalable-based on ions manipulated by lasers and multi-zone traps. Division researchers also demonstrated a robust, high-fidelity logic gate based only on changes of phase in a two-ion ${ }^{9} \mathrm{Be}^{+}$system. (See Fig. 7.)

Recently, Division researchers demonstrated deterministic quantum teleportation of qubits in a three-ion trap. A coherent superposition of two internal states was generated in one of the ions, and then the quantum states were teleported to a second ion through a third, intermediary ion. This experiment and a similar one simultaneously reported by a group from Innsbruck were the world's first demonstrations of teleportation of massive particle qubits, rather than pho-

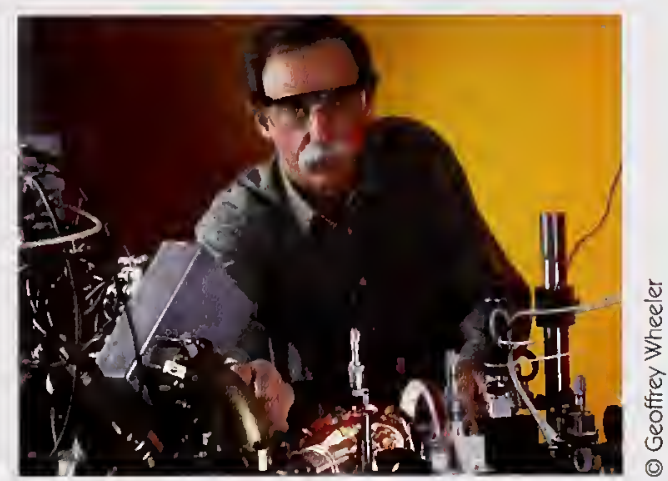

Figure 7. David Wineland adjusting one of the systems used for studying quantum-logic gates.

ton qubits. Quantum teleportation could be crucial to realization of a largescale quantum computer, enabling rapid transmission of qubit information throughout the computer without the need to physically move qubits.

CONTACT: DR. DAVID J. WINELAND (303) 497-5286

wineland@boulder.nist.gov 
GOAL

TO MAKE

TRANSFORMATIONAL

ADVANCES AT THE FRONTIERS

OF MEASUREMENT SCIENCE,

IN PARTNERSHIP WITH THE

UNIVERSITY OF COLORADO

AT JILA

\section{QUANTUM PHYSICS DIVISION}

The strategy of the Quantum Physics Division is to belp produce the next generation of scientists and to investigate new ways of precisely directing and controlling light, atoms, and molecules; measuring electronic, chemical, and biological processes at the nanoscale; and manipulating ultrashort light pulses.

\section{he first strategic element}

is to develop precision

measurement tools and

applications.

\section{Precision}

\section{Measurement}

\section{INTENDED OUTCOME AND \\ BACKGROUND}

The Quantum Physics Division continues a leadership role in developing new precision-measurement techniques and applications. Precision measurement is used for many industrial and scientific purposes, and is central to many activities of NIST and other standards laboratories. Applications range from providing the length scale for mechanical measurements to providing a direct connection between optical and radio frequencies.
The highly stabilized laser, a Division specialty, is the workhorse of precision measurement. Traditionally, this meant continuous wave (CW) lasers that generated light with a very precise and stable single frequency. Recently, the Division became an international leader in adapting CW techniques to the stabilization of mode-locked lasers. These generate wideband "comb" spectra, consisting of long series of sharp spectral lines. Each such line can have individually a very precise and stable frequency, with their frequency separation constrained to be the repetition rate of the laser. This rate may be locked to a radio frequency $(R F)$ reference (e.g., microwave).

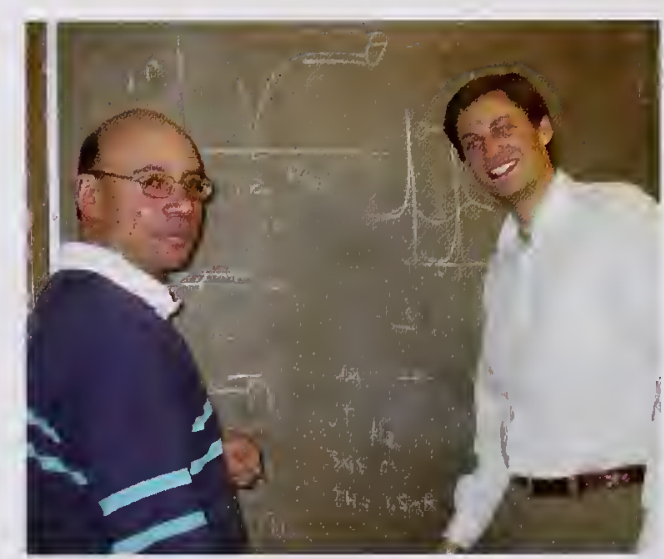

Figure 1. The Quantum Physics Division's newest hires, Ralph Jiminez (1) and Konrad Lehnert ( $r$ )
Frequency combs significantly advance the art of measuring optical frequencies and establish a direct connection between optical and radio frequencies. This connection enables optical atomic clocks and absolute optical frequency metrology.

\section{ACCOMPLISHMENTS}

\section{Advances in Frequency Comb Technology}

Early progress in comb techniques was enabled by the development of microstructure optical fiber, which combines high nonlinearity with low dispersion to allow strong nonlinear broadening of weak pulses. It broadened the output of mode-locked lasers to the point where the spectra included comb lines that were twice the frequency of other comb lines-a full octave, in musical terms. However, microstructure fiber has significant drawbacks, including coupling into its $2 \mu \mathrm{m}$ core, drift of the coupling over time, facet damage, and amplitude-to-phasenoise conversion. 
To eliminate the need for microstructure fiber, we built a mode-locked Ti:sapphire laser that directly generates an octave-spanning spectrum. We demonstrated that its offset frequency could be locked using self-referencing.

Self-referencing has been the principal technique used to control the offset frequency of the comb. One compares the high- and low-frequency wings of an octave-spanning comb, and frequencylocks a line with its double. It is typically implemented as a bulk-optic interferometer.

Recently, we have measured and controlled the offset frequency using quantum interference of injected photocurrents in a semiconductor. This technique uses quantum rather than optical interference. It replaces a bulk optical interferometer with a single semiconductor chip, which is smaller, less expensive, and less complicated. The phase stability, as measured by an outof-loop, standard self-referencing interferometer, was as good, or better, than that achieved using the standard selfreferencing technique.

CONTACT: DR. STEVEN T. CUNDIFF (303) 492-7858 cundiffs@jila.colorado.edu
New Implementation of an Optical Molecular Clock

An optical "atomic" clock uses as its reference an optical frequency transition that has a lower relative frequency uncertainty than does the standard, cesium microwave frequency transition. An optical clock phase-coherently derives an RF signal from the optical frequency standard, which can be done with a mode-locked laser.

We have built an optical molecular clock, based on difference-frequency generation that does not rely on an octave-spanning spectrum, microstructured fiber, additional CW lasers, or stabilization of the offser frequency. We stabilize a HeNe laser at $3.39 \mu \mathrm{m}$ wavelength using the methane F2(2) line. This laser serves as the infrared optical frequency standard to which the repetition rate of a Ti:sapphire laser is phase-coherently locked. We use the mode-locked laser's frequency comb for difference-frequency generation to provide an infrared comb at $3.39 \mu \mathrm{m}$ with a null carrier-envelope offset. This infrared comb phase-coherently links the $88 \mathrm{THz}$ optical reference and the repetition rate.

Comparison of the repetition rate signal with a second frequency comb stabilized to molecular iodine shows an instability of $0.12 \mathrm{pHz} / \mathrm{Hz}$ at $1 \mathrm{~s}$, limited by microwave detection of the repetition rates. The single sideband phase noise of the microwave signal, for a $1 \mathrm{GHz}$ carrier frequency, is below $-93 \mathrm{dBc} / \mathrm{Hz}$ at $1 \mathrm{~Hz}$ offset.

CONTACT: DR. JUN YE

(303) 735-3171

ye@jila.colorado.edu
Novel Spectroscopy of Cold Atoms

Historically, stabilized CW lasers have been integral to spectroscopy; frequency combs from mode-locked lasers served as reference rulers for wavelength.

However, frequency combs also enable vastly improved measurement of atomic and molecular structural information. The combination of frequency domain precision with time domain dynamics establishes a new paradigm for spectroscopy, connecting the fields of precision measurement and ultrafast science.

We recently demonstrated direct, precision spectroscopy of global atomic structure using a single, stabilized optical frequency comb. This approach exploits massively parallel spectral probing in the frequency domain. The widebandwidth, phase-coherent, absolutefrequency-referenced comb allowed us to precisely measure atomic energy level structure in the optical, terahertz, and radio frequency domains at the same time, with a systematic-error-free connection among all spectral features. Furthermore, the pulsed excitation permits time-resolved studies of dynamics and real-time monitoring and control of both optical and quantum-coherent interactions and state transfer.

In a real sense, we have merged the fields of precision spectroscopy and coherent control. At short times, we monitor and control coherent accumulation and population transfer. At long times, we recover all the information pertinent to atomic level structure at a resolution limited only by the atomic linewidth. The spectroscopic precision we have achieved with a frequency comb rivals that of the state-of-the-art CW laser-based approaches, even 

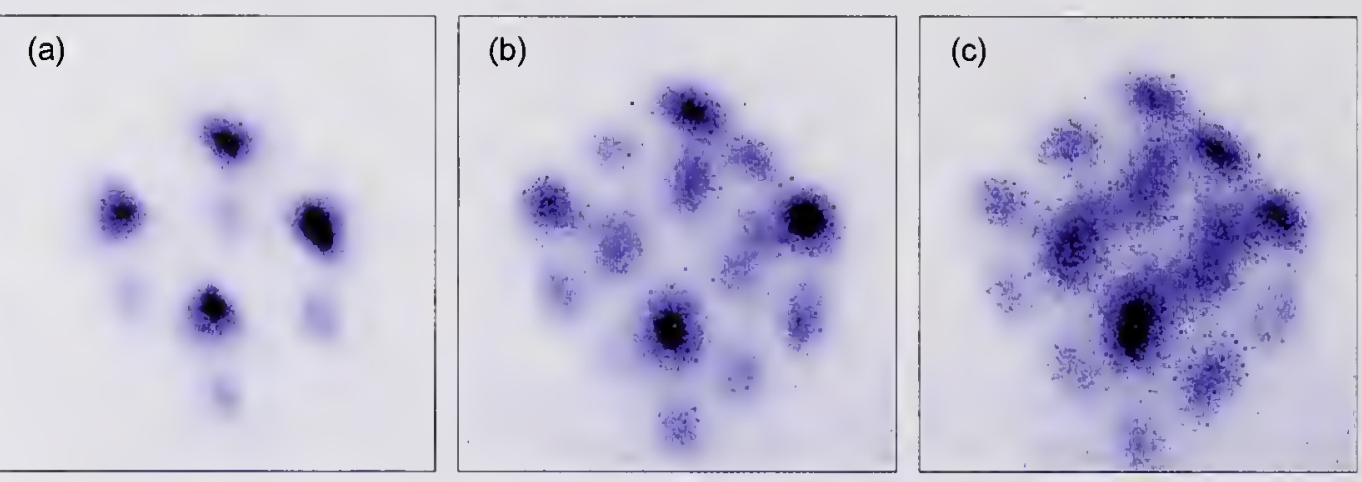

Figure 2. Images showing strontium atoms forming a "cube" as the frequency of the laser light used to manipulate them changes. (a) Atoms become visible at the eight corners of a cube. (b) Atoms also appear at the midpoints of the cube edges and begin appearing at the center of each face. (c) Atoms appear more clearly at the centers of each cube face.

though our measurement covers a frequency range of hundreds of terahertz with a single laser.

Additionally, we have experimentally demonstrated the frequency comb's mechanical action on cold atoms. Precise control of the frequency and phase of the entire comb spectrum allows us to apply a light force on probed atoms without accumulating significant effects from heating or Doppler shifts. We exploit this mechanical cooling for spectroscopy, significantly reducing systematic errors. The flexibility in the comb structure also allows us to carefully measure and control the AC Stark shift inevitably present in light-atom interactions.

Our research on ultracold strontium atoms has three main thrusts: optical atomic clocks, ultracold collisions, and quantum degeneracy. Thus far, we have obtained new insights to laser-cooling mechanisms by using the nuclear-spin magnetic degeneracy of cold $\mathrm{Sr}$ atoms to obtain strong sub-Doppler cooling, despite a closely overlapped excited state manifold.
We have demonstrated several unique aspects of a magneto-optic trap supported by narrow-line cooling, including achieving a subrecoil temperature of $250 \mathrm{nK}$ (the recoil temperature is $450 \mathrm{nK})$ for the first time without needing atomic coherence in the ground state. At such low temperatures, the atom cloud displays interesting dynamics. For example, if we tune the trapping laser frequency above the atomic resonance, the cloud divides into discrete momentum packets resembling lattice sites on a face-centered-cubic crystal. (See Fig. 2.)

Precision spectroscopy on ultracold Sr has also begun. We have determined the absolute frequency of the $\mathrm{Sr}$ transition to a $25 \mathrm{~Hz}$ uncertainty. We have also observed a densiry-related frequency shift and linewidth broadening for alkaline earth atoms. This will be valuable information for understanding the collision dynamics of these atoms in a quantitative manner.

CONTACT: DR. JUN YE

(303) 735-3171

ye@jila.colorado.edu

\section{Fast, Ultrasensitive}

\section{Bolometers and Calorimeters}

The emerging fields of quantum computing and quantum cryptography require fast, energy-resolving photon counters (calorimeters). At the same time, future space-based observatories will require imaging arrays of thousands of ultrasensitive bolometers. It is impractical to read-out each of these sensors with its own amplifier. Thus, the ability to multiplex the sensors, i.e., to read-out many sensors with one amplifier, is paramount.

We have built sensors that can be configured as either fast calorimeters or sensitive, multiplexible bolometers. The incident power or energy is inferred from the resistance of a nanoscale thermistor formed from normal metal-insulator-superconductor junctions.

Recent measurements show that our sensor is fast enough to count photons in submicrosecond times and sensitive enough to detect an incident power of $7 \times 10^{-17}$ watts in one second, when operated at $270 \mathrm{mK}$.

The key innovation relies on measuring the temperature-dependent resistance using a microwave technique compatible with subkelvin operating temperatures. The sensors can be configured either for maximum speed, in which case they are calorimeters, or for slower, multiplexible operation, in which case they are sensitive bolometers. By optimizing the sensors and operating them at a temperature of $50 \mathrm{mK}$, we anticipate bolometric sensitivities of $10^{-20} \mathrm{~W} / \mathrm{Hz}^{1 / 2}$, meeting the requirement of the most demanding future space missions. 


\section{Gravity Measurements}

We measure the Newtonian constant of gravity, $G$, and the acceleration of gravity, $g$. To measure $G$, we directly compare the attraction of a laboratory mass to the attraction of the Earth. Our experiment translates length changes due to gravity into measurable beat frequency changes between two lasers. We expect our final measurements to yield a relative uncertainty for $G$ of about $4 \times 10^{-5}$.

We are also developing a new, compact instrument to measure the acceleration of gravity, $g$. The instrument employs a novel cam-based mechanism to obtain extremely fast and quiet control of the entire release, freefall (measure), catch, and lift cycle. It can be used for volcanology and precision-measurement studies, and has an uncertainty of approximately $3 \mu \mathrm{Gal}$ (relative uncertainty of $3 \times 10^{-9}$ ).

CONTACT: DR. JAMES E. FALLER

(303) 492-8509

fallerj@jila.colorado.edu

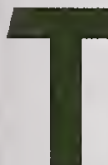

he second strategic element is to exploit BoseEinstein condensation, quantum degenerate Fermi gases, and cold molecules for metrology and ultralowtemperature physics.

\section{Ultracold Atoms and Molecules}

\section{INTENDED OUTCOME AND \\ BACKGROUND}

The Quantum Physics Division and JILA are world renown for studies of Bose-Einstein condensates and quantum degenerate Fermi gases. The exemplary JILA collaboration between NIST and the University of Colorado (CU) led to the achievement of the first BoseEinstein condensate by Eric Cornell (NIST) and Carl Wieman (CU), who together received the 2001 Nobel Prize in Physics. This achievement, coupled with the creation of the first quantum degenerate Fermi gas and the first Fermi condensate by MacArthur Prize-winner Deborah Jin (NIST), places the Quantum Physics Division and JILA at the forefront of studies of macroscopic quantum mechanical systems.

A better understanding of these systems is critical as the miniaturization of electronic components pushes in to the size region where quantum mechanical effects play a significant role in their operation. Additionally, these systems provide unique opportunities for metrology and for gaining insights into analogous transitions in technologically important solid-state systems. We plan to continue to explore the new quantum mechanical systems that these discoveries have made accessible and to maintain our leadership position. The development of techniques to produce ultracold molecules also promises important advances in chemical physics.

\section{ACCOMPLISHMENTS}

\section{BEC on a Chip}

We have succeeded in creating an atom interferometer on a lithographically patterned microstructure, an "atom chip." The long-term technical goal of this work is to develop supersensitive and compact inertial sensors, e.g., gyroscopes and gravity gradiometers.

A condensate of ultracold atoms is guided along an invisible magnetic track generated by currents carried in tiny wires. The condensate is split coherently into two parts that travel a short distance in opposite directions along the track. Then the directions are reversed, the two parts of the condensate recombine, and we observe patterns of high and low density-atom-wave interference fringes. The patterns of these fringes are sensitive to any acceleration felt by the chip during the period when the two parts of the condensate were separated.

In this first realization, the sensitivity is limited by the relatively short time the condensate parts remain separated. Future work will concentrate on improving performance.

CONTACT: DR. ERIC A. CORNELL (303) 492-6281 cornell@jila.colorado.edu 


\section{Destroying BEC}

Ironically, one of the more important scientific advances in our recent ultracold research is progress towards destroying a Bose-Einstein condensate. As a ball of Bose-condensed gas starts to rotate, it becomes pierced by progressively more tiny tornadoes known as quantized vortices. When the rotation rate is very high, these vortices organize themselves into a tightly packed triangular array. At the highest level of rotation, the size of each vortex becomes large compared to the spacing between them.

Theory predicts a reordering transition in which the condensate is destroyed and replaced by a highly correlated gas, reminiscent of the quantum Hall state in solids. Achieving and characterizing this transition is a prime scientific goal because we believe it will generate important insights into analogous transitions in technologically important, condensed-matter systems, such as the giant-magnetoresistance phenomenon that is used in disk-drive read-sensor technology.

In the last two years we have come much closer to achieving our goal. We have installed high-intensity lasers that we hope will soon bring us even closer.

CONTACT: DR. ERIC A. CORNELL

(303) 492-6281

cornell@jila.colorado.edu

\section{Cold Fermions and}

\section{Resonance Superfluidity}

Our work on cold Fermi gases has progressed at a rapid pace. In January 2004, we reported the first observation of a "Fermi condensate." This novel phase of matter was predicted in 2001 by JILA's Murray Holland $(\mathrm{CU})$, who coined the term "resonance superfluidity" because of the key role of a magnetic-field Feshbach resonance in creating these condensates of fermionic-atom pairs.

Our experimental observation, which included a measurement of the temperature and magnetic-field phase diagram, revealed a surprisingly high transition temperature (relative to the Fermi temperature). It triggered a great deal of experimental and theoretical work exploring this new Fermi superfluid phase. Because of the unique control we have in ultracold gas systems, future studies of the Fermi condensate have the potential for dramatically enhancing our understanding of the connection between superconductivity and Bose-Einstein condensation.

Progress leading up to the Fermi-condensate observation yielded a number of important advances, including the first spectroscopic measurements of the interaction energy in a Fermi gas near a Feshbach resonance, the first reversible conversion (pairwise) of the majority of trapped ultracold atoms into ultracold molecules, and a direct measurement of the binding energy of these molecules with the introduction of RF photodissociation.

CONTACT: DR. DEBORAH JIN

(303) 492-0256

jind@jila.colorado.edu

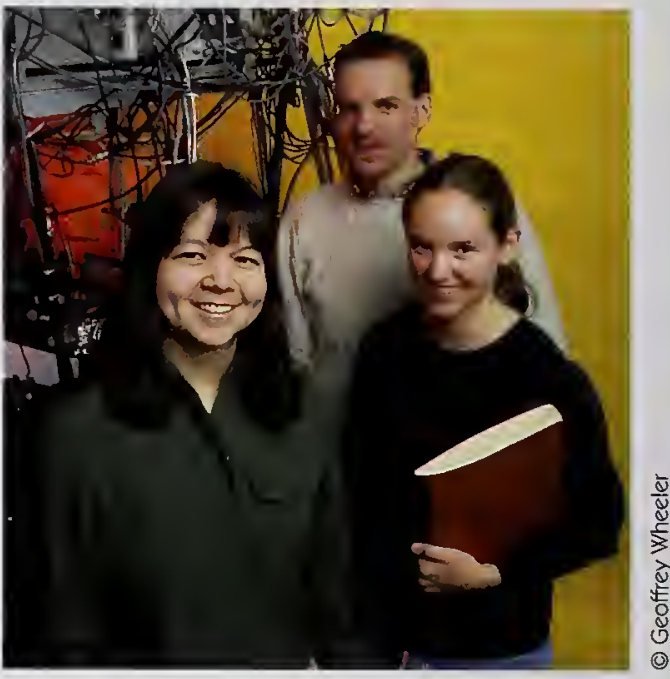

Figure 3. MacArthur Prize-winner Deborah Jin, with her CU graduate student Cindy Regal and CU postdoctoral research associate Marcus Greiner.

\section{Bose-Fermi Mixtures}

We are now exploring Bose-Fermi mixtures. We employ sympathetic cooling to produce Bose-Einstein condensates of ${ }^{87} \mathrm{Rb}$ atoms simultaneously trapped with a Fermi gas of ${ }^{40} \mathrm{~K}$ atoms.

Focusing on interactions in the gas mixture, we perform collision studies to measure the magnitude of the interspecies scattering length between the ${ }^{87} \mathrm{Rb}$ and ${ }^{40} \mathrm{~K}$ atoms. This single parameter characterizes the interactions between the boson and fermion atoms and thus controls many of the equilibrium and dynamic properties of the ultracold gas mixture. By using an optical dipole trap and manipulating the atoms' spin states, we recently observed four heteronuclear Feshbach resonances.

These resonances open up the possibility for controlling the interactions in the gas. They also provide a means for creating ultracold, weakly bound heteronuclear molecules.

CONTACT: DR. DEBORAH JIN

(303) 492-0256

jind@jila.colorado.edu 
he third strategic

element is to advance

ultrafast science.

\section{Ultrafast Science}

\section{INTENDED OUTCOME AND}

BACKGROUND

Ultrafast science has traditionally exploited ultrashort optical pulses with durations as short as a few femtoseconds. These pulses provide precise time resolution and/or high peak powers. Because they are produced by modelocked lasers, their frequency comb spectra can be controlled with the techniques described in the first strategic element. In ultrafast science, the control of the comb spectrum corresponds to control of the electric field of the pulses in the time domain. Control of the electric field of the pulses enables the observation of unique physical phenomena and the ability to coherently synthesize new pulse shapes.

Traditional ultrafast techniques are used in the Division to study the dynamics of electron spins in semiconductors and of biomolecules, providing technologically important information for both optoelectronics and biotechnology. We use phase stabilization to improve measurement techniques and to develop radical new ultrafast technologies, such as "gainless" amplification of femtosecond pulses in a buildup cavity.

\section{AC COMPLISHMENTS}

\section{Passive Optical Cavity \\ Interactions with a \\ Femtosecond Comb}

We have directly stabilized a modelocked laser's optical frequency comb to a high-finesse passive optical cavity. We made detailed comparisons of two stabilization schemes to optimize cavity stabilization and to develop approaches for overcoming limitations on transfer of the cavity's frequency stability to the microwave domain. We explored the stability of the frequency comb in both the optical and the radio frequency domains.

With an independent, stable CW laser, we verified that the linewidth and stability of the frequency comb components are $<300 \mathrm{~Hz}$ and $50 \mathrm{fHz} / \mathrm{Hz}$ at $1 \mathrm{~s}$ averaging time, respectively, both limited by the CW laser. Such performance represents the state of the art in frequency and phase stabilization of a modelocked laser. It confirms that a highly stable, passive optical cavity can directly stabilize the repetition frequency and carrier-envelope phase of ultrashort pulses to a level rivaling that achieved by CW lasers.

We also demonstrated a technique for enhancing femtosecond pulses from a pulse train via coherent buildup in a high-finesse cavity. By periodically extracting the intracavity pulse, we increased the net pulse energy 42 -fold to 70 -fold. Pulse energies of $>200 \mathrm{~nJ}$ were demonstrated. Greater single-pulse amplification is achieved using higher finesse cavities at the expense of a reduced repetition rate.
We also developed a measurement protocol for mirror dispersion. The measurement accuracy is more than ten times better than the previous state-ofthe-art, white light interferometry technique. We provided these precise measurements to the mirror manufacturer, enabling them to significantly improve their ability to make large-bandwidth, low-loss, and low-dispersion mirrors.

CONTACT: DR, JUN YE

(303) 735-3171

ye@jila.colorado.edu

\section{Spin Coherence in}

\section{Semiconductors}

The possibility of using electrons' spin degree-of-freedom for encoding information has attracted significant attention. This technology could lead to devices analogous to traditional microelectronics or to others based on quantum information concepts.

Optical techniques are currently the best way to prepare and probe spin-coherent states, as true "spintronic" devices are still very primitive. Optical preparation and probing are also likely to be preferred for quantum information applications.

We are acquiring a basic understanding of how optical excitations in semiconductors manifest themselves in traditional optical techniques that excel at measuring the $g$-factor, which determines how the electron precesses and its spin coherence time, which limits duration of any operation. However, interpretation of the results requires knowledge of the different optical excitations created by the incident pulses. In semi- 
conductors, these include free electronhole pairs, excitons (bound electron-hole pairs), biexcitons (two excitons bound together, analogous to a hydrogen molecule), and trions (an exciton bound to an electron). Trions are particularly important because they occur in materials containing excess electrons, which can have long spin coherence times.

We are exploring ways to control the number of excess electrons to determine why their presence increases the spin coherence time. The most promising technique is the use of special quantum wells, where the spatial separation of the electrons and holes results in a very long recombination time, which means that significant densities can be achieved with modest powers.

CONTACT: DR. STEVEN T. CUNDIFF (303) 492-7858 cundiffs@jila.colorado.edu

\section{Optical Two-Dimensional}

\section{Fourier-Transform}

\section{Spectroscopy of Excitons}

The concept of multidimensional Fourier-transform spectroscopy was originally developed in nuclear magnetic resonance. This powerful technique excels at elucidating coupling between resonances.

We have developed an optical twodimensional Fourier-transform spectrometer to study coupling between optical excitation in semiconductors, specifically between excitons and electron-hole pairs. The results are providing insight into the many-body physics that underlies the interactions between the carriers. Specifically, it allows a direct mapping of the real and imaginary parts of the self-energy. The selfenergy is a fundamental quantity that can be directly calculated using manybody theory, which, in turn, is used to model optoelectronic devices such as diode lasers.

CONTACT: DR. STEVEN T. CUNDIFF (303) 492-7858

cundiffs@jila.colorado.edu

he fourth strategic

element is to investigate

biological systems at the

single-molecule level.

\section{Biophysics}

\section{INTENDED OUTCOME AND BACKGROUND}

The Quantum Physics Division investigates important biological systems at the single-molecule level, thus leveraging our measurement expertise and experience with atomic and quantum systems. Accordingly, we are evolving a part of our research program in this direction to help NIST contribute to the scientific revolution taking place in biophysics.

The Division's strengths are the foundation of our efforts to contribute to groundbreaking research on single biomolecules. These strengths include our ability to build institutional bridges to renowned university departments, a superlative infrastructure, experience in manipulating and measuring atomic and quantum mechanical systems, and a reputation that attracts the best and brightest of today's young scientists. Our new biophysics program is being implemented in close collaboration with the Department of Molecular, Cellular, and Developmental Biology, and the Biochemistry Division of the Department of Chemistry, at the University of Colorado. By integrating additional departments into JILA, we will enhance the very productive, interdisciplinary character of the institute. Most importantly, because of our existing expertise, our biophysics program should rapidly build on the successes achieved by our other programs.

\section{ACCOMPLISHMENTS}

\section{Single-Molecule Fluorescence Microscopy: Biomolecular Conformational Dynamics}

This biometrology project probes conformational dynamics (e.g., folding and unfolding) of biomolecules in chemically active states. We focus on simplifying complex RNA structures to understand the mechanisms that stabilize specific structural folds. This information is crucial to understanding RNA-based enzymes, or ribozymes.

We use ultrasensitive time-, color-, and polarization-resolved fluorescence detection of single RNA molecules in a confocal microscope. A mode-locked laser is focused into an aqueous, dilute sample of RNA molecules, with less than a single molecule in the detection region. The resulting weak fluorescence is sorted by both polarization and color, and is imaged onto single-photon-counting avalanche photodiodes. The individual photon events are recorded as a function 
of time after the incident laser pulse, achieving an 11 order-of-magnitude range of kinetic time scales.

The fast-fluorescence behavior monitors the local environment of the RNA and can be extracted via time-correlated, single-photon counting. Using fluorescence resonant energy transfer (FRET), we can measure distances $(2 \mathrm{~nm}$ to $8 \mathrm{~nm}$ ) between specifically labeled sites on the RNA. This allows us to investigate the folding kinetics for RNA in real time at the single-molecule level.

Thus far, studies have required tethering the RNA to a glass coverslip. To eliminate possible surface effects, we are developing methods for studying "free" RNA by exploiting "burst-mode" singlemolecule microscopy. This technique detects species diffusing into and out of the confocal region, but it limits the time that we can probe single biomolecules. Consequently, we are also working on methods to slow down diffusion. We plan to isolate single RNA molecules in liposomes, which could be manipulated by optical tweezers and viewed microscopically for arbitrary lengths of time.

CONTACT: DR. DAVID NESBITT

(303) 492-8857

djn@jila.colorado.edu

\section{Single-Biomolecule}

\section{Electrophoresis}

We have begun exploring single-molecule electrophoresis. The apparatus for these studies is based on wide-field microscopy through a thin gel-electrophoresis cell. In this technique, weak electric fields are used to coax single DNA molecules through a micrometer-scale field of view.
The DNA motion can be studied by labeling it with highly fluorescent dyes that are illuminated by a laser and detected by imaging on an intensified $\mathrm{CCD}$-array camera. This method is sensitive enough to image at a $10 \mathrm{~Hz}$ frame repetition rate, and allows us to monitor single-DNA electrophoresis dynamics in real time.

We can automatically determine the location of the DNA as a function of time, and thereby track its progress and mobility during electrophoresis. These studies permit direct visualization and detailed tests of biopolymer percolation dynamics. In the future, they will help us to learn more about improving separation efficiency and the kinetics/ dynamics of protein-DNA binding. The latter are relevant to cell regulatory processes and would be amenable to study at the single-molecule level.

\section{CONTACT: DR. DAVID NESBITT \\ (303) 492-8857 \\ djn@jila.colorado.edu}

\section{Single-Molecule \\ Measurement with \\ Nanometer Resolution}

We are studying molecular motors at the level of single molecules. Our efforts are prompted by the insight that motor proteins generate measurable force and displacement.

Determining the elementary step size of motor proteins is crucial to understanding their mechanisms. The enzymes involved in DNA replication, DNA transcription, and RNA translation work, in a literal sense, as molecular motors; once bound to their nucleic acid substrates, they translocate in a more-or-less unidirectional fashion,

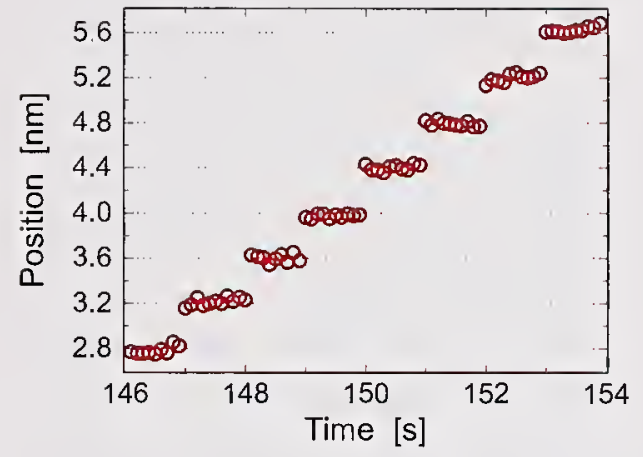

Figure 4. Individual $0.4 \mathrm{~nm}$ steps measured in a new differential back-focal-plane detection, optical-trapping microscope. The fact that the data points lie on lines demonstrates that $0.1 \mathrm{~nm}$ stability is achieved over several seconds.

carrying out hundreds to millions of enzymatic cycles.

We have recently developed an instrument with subnanometer spatial resolution that has significantly improved the displacements that optical trapping experiments can detect. (See Fig. 4.) Besides studying DNA-based molecular motors, such as helicases, we are using our new subnanometer technology to study transcription factors, which bend DNA rather than moving along it. Transcription factors turn on and off the production of messenger RNA and thereby protein expression.

We are also starting a new program to study the unfolding of single molecules of RNA. This work will bring together JILA's expertise in laser and precision measurement with CU's expertise in RNA research.

CONTACT: DR. THOMAS T. PERKINS (303) 492-5291

tperkins@jilau1.colorado.edu 


\section{$(3)$ \\ TO COORDINATE \\ AND FACILLATE THE \\ ELECTRONIC \\ DISSEMINATION OF \\ INFORMATION VIA THE \\ INTERNET}

\section{OFFICE OF ELECTRONIC COMMERCE IN SCIENTIFIC AND ENGINEERING DATA}

The strategy for meeting this goal is to publish Physics Laboratory informa-

tion on the World Wide Web, to develop web-accessible databases of physical

reference data, and to evolve protocols to ensure interoperability in the

exchange of scientific and engineering data.

\section{Dissemination of Information}

\section{INTENDED OUTCOME AND}

\section{BACKGROUND}

The Office of Electronic Commerce in Scientific and Engineering Data (ECSED) is responsible for the Physics Laboratory (PL) World Wide Web pages, at physics.nist.gov. We produce material for Web publication, encourage and support the production of material by others, and ensure the high quality of disseminated information. We are also engaged with PL Divisions and the NIST Standard Reference Data Program in developing physical reference databases for Web dissemination. We design and develop effective Web database interfaces to facilitate access to the data, providing data in multiple formats suitable for customer needs.

Since June 1994, we have provided a wide array of information ranging from physical reference data, technical activities, research and calibration facilities, technical contacts, publication lists, general interest, and news items. For the most recent six months, there was an average of $1 \frac{1}{2}$ million requests for web pages per month from the Gaithersburg server (nearly half from about 30 online databases containing physical reference data). A complete list of our databases is available at http://physics.nist.gov/data.

Recent work includes the development of new (1-5) and updated (6-11) Web databases:

1. Handbook of Basic Atomic Spectroscopic Data

2. Energy Levels of Hydrogen and Deuterium

3. Spectral Data for the Chandra X-Ray Observatory

4. X-Ray Transition Energies

5. Potential Energy Surface Database of Group II Dimer Molecules

6. Fundamental Physical Constants

7. Searchable Bibliography on the Constants

8. NIST Atomic Spectra Database

9. Electron-Impact Cross Sections for Ionization and Excitation Database

10. X-Ray Form Factor, Attenuation, and Scattering Tables

11. Frequencies for Interstellar Molecular Microwave Transitions
ACCOMPLISHMENTS

\section{Units Markup Language}

We have continued to develop an XML (eXtensible Markup Language) schema for encoding scientific measurement units. Adoption of this schema will allow the unambiguous exchange of numerical data over the Internet. We have established collaborations with Lawrence Berkeley National Laboratory, Mathsoft, an engineering software company, and SEMATECH, a consortium of the semiconductor industry.

To complement the UnitsML schema, we are in the process of developing an XML repository containing detailed information on both SI and non-SI scientific units. We anticipate this repository will be used by our customers to download industry-specific dictionaries of scientific units. Development of the UnitsML schema, repository, and a general ontology for scientific units is supported in part by the NIST Systems Integration for Manufacturing Applications (SIMA) program.

CONTACT: DR. ROBERT DRAGOSET

(301) $975-3718$

robert.dragoset@nist.gov 


\section{Support for ITL on the DLMF}

We are providing support to the Mathematical and Computational Sciences Division of the NIST Information Technology Laboratory (ITL) to develop the Digital Library of Mathematical Functions (DLMF). Abramowitz and Stegun's Handbook of Mathematical Functions (with Formulas, Graphs, and Mathematical Tables) was first published by the National Bureau of Standards in 1964. It remains a technical bestseller and is among the most widely cited of all math reference compendia. But the Handbook is increasingly out-of-date, especially its numerical tables which account for over half its length. It also lacks the many recent developments in the theory and computation of functions that find essential use in physics and other sciences. A project is underway to develop a replacement, which should become a major resource of math reference data for special functions and their applications. The new $D L M F$ will appear in a hardcover edition and as a free electronic publication on the Web.

The Office of ECSED has a strong capability in LaTeX, a powerful, complex typesetting language particularly useful for representing mathematical

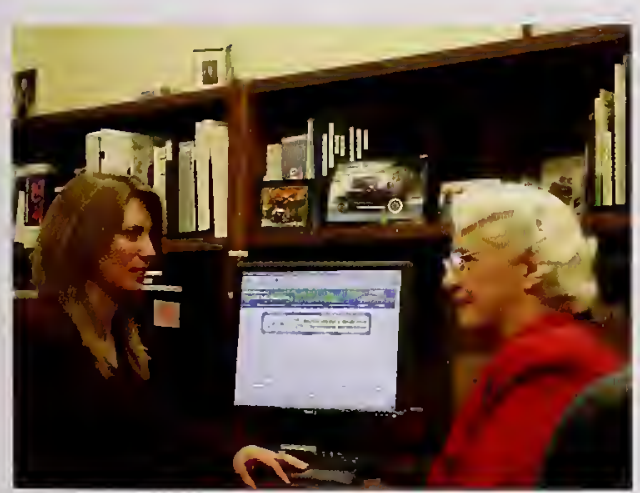

Figure 1. Karen Olsen and Gloria Wiersma discuss the design of an online database expressions. Since the $D L M F$ is being prepared in LaTeX, we are providing support to ITL on the preparation of this publication. This entails editing existing chapters with extensive handwritten corrections, in addition to adding new sections from handwritten text. We began working on the DLMF in August 2003 (providing up to $40 \%$ of one staff person per year) and have thus far worked on 23 of the 40 chapters. The hardcover edition will be over 1000 pages in length and is scheduled to be printed in 2005 .

\section{CONTACT: GLORIA WIERSMA} (301) 975-5547

gloria.wiersma@nist.gov

\section{New Capabilities for On-Line Databases}

The Electron-Impact Cross Sections for Ionization and Excitation Database has been available on the Web since August 1997. We recently collaborated with the Atomic Physics Division to update the database and release Version 3.0.

Additions to the database include: excitation cross sections for atoms $\mathrm{H}, \mathrm{He}$, and $\mathrm{Li}$; and ionization cross sections for atoms $\mathrm{B}, \mathrm{C}, \mathrm{N}, \mathrm{O}, \mathrm{Al}, \mathrm{Ga}$, and $\mathrm{In}$, and for molecules $\mathrm{C}_{2} \mathrm{H}_{3}, \mathrm{C}_{3} \mathrm{H}_{3}, \mathrm{C}_{3} \mathrm{H}_{4}$ (allene and propyne), $\mathrm{C}_{3} \mathrm{H}_{5}, \mathrm{C}_{3} \mathrm{H}_{6}$, $\mathrm{C}_{4} \mathrm{H}_{2}, \mathrm{C}_{4} \mathrm{H}_{4}, \mathrm{C}_{4} \mathrm{H}_{6}, \mathrm{C}_{4} \mathrm{H}_{8}$ (1-butene, trans-2-butene, and isobutene), $\mathrm{C}_{6} \mathrm{H}_{2}$, $\mathrm{C}_{6} \mathrm{H}_{6}$ (fulvene), $\mathrm{SiF}_{4}, \mathrm{HO}_{2}$, and six chlorofluorocarbons $\left(\mathrm{CClF}_{3}, \mathrm{CCl}_{2} \mathrm{~F}_{2}\right.$, $\left.\mathrm{CCl}_{3} \mathrm{~F}, \mathrm{CHClF}_{2}, \mathrm{CHCl}_{2} \mathrm{~F}, \mathrm{CH}_{2} \mathrm{ClF}\right)$. The user interface has been significantly enhanced, and, to accommodate the extensive additions of data, atomic and molecular data are now accessible through separate interfaces.

With the addition of cross section data for atoms and the molecules, we are reaching a much wider customer base. The database will benefit those working in the areas of fusion plasma modeling, plasma processing of materials, modeling of semiconductor fabrication, and modeling of combustion chemistry useful to the automotive industry. The Electron-Impact Cross Sections for Ionization and Excitation Database can be accessed on the Web at http://physics.nist.gov/ionxsec

This updated database was developed using two popular Web development tools: MySQL and PHP. MySQL is a relational database management system. PHP, a widely used general-purpose scripting language, provides interfaces to many different database systems and has built-in libraries for many common Web tasks. Both PHP and MySQL have the strengths of high performance, low cost, ease of use, efficiency and portability; and they are Open Source solutions.

Using MySQL and PHP, we have created a Web-based data entry form so that scientists can now add data to the database. The data retrieval interface (a periodic table and various selection forms) is dynamically generated based on the current contents of the MySQL database. Thus, when data is entered into the MySQL database, it is immediately viewable.

\section{CONTACT: KAREN OLSEN \\ (301) 975-3286 \\ karen.olsen@nist.sov}


Service to America

Medal

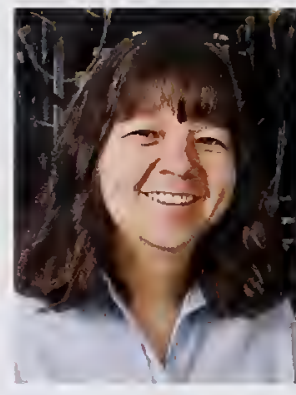

Deborah S. Jin,

Quantum Physics

Division, was

awarded a 2004

Service to America

Medal by Govern-

ment Executive

Magazine and the

Partnership for Public Service. She was recognized for significant contributions to the nation in activities related to science and the environment. The award was for creating "a new form of matter which could potentially unlock the key to superconductivity, a phenomenon with the potential for improving energy efficiency dramatically across a broad range of applications."

\section{MacArthur Fellowship}

Deborah S. Jin, Quantum Physics Division, was also a 2003 winner of a MacArthur Fellowship, commonly known as the "genius grant." The MacArthur Fellows Program awards unrestricted fellowships to individuals who have shown extraordinary originality and dedication in their creative pursuits and a marked capacity for self-direction.

\section{Academician of the Pontifical Academy of Sciences}

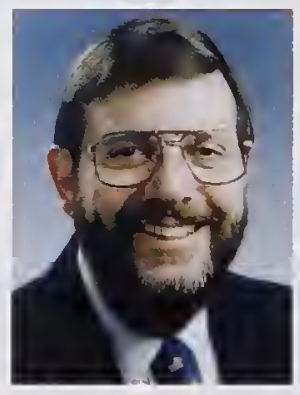

William D. Phillips, Atomic Physics Division, was appointed a 2004 Academician of the Pontifical Academy of Sciences. He was recognized for his contributions to science and for his Christian character, dedication, and example. The Pontifical Academy of Sciences was founded in Rome in 1603 with the name Academy of the Lynxes and led by Galileo Galilei. It is currently made up of 80 pontifical academicians named for life by the Pope after having been proposed by the academicians themselves.

\section{Davisson-Germer Prize}

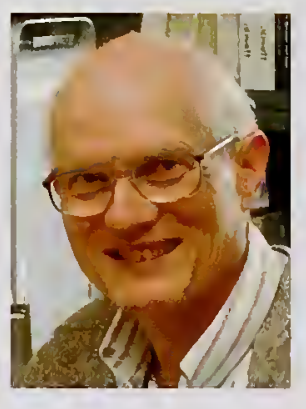

Paul S. Julienne,

Atomic Physics

Division, received

the 2004 Davisson-

Germer Prize in

Atomic or Surface

Physics from the

American Physical

Society. He was recognized for his pioneering studies of the theory of ultracold atomic collisions and its applications to precision metrology and quantum gas dynamics. The Prize was established in 1965 by AT\& $\&$ T Bell Laboratories (now Lucent Technologies) as a means of recognizing outstanding scientific work in America.

\section{Frederick Ives Award /Jarus W. Quinn Endowment}

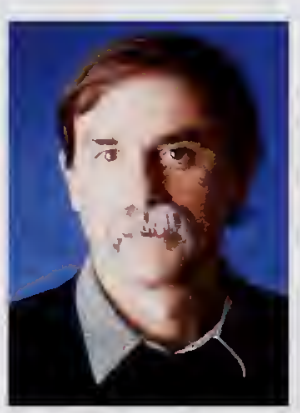

David J. Wineland, Time and Frequency Division, received the 2004 Frederick Ives Award/Jarus W. Quinn Endowment of the Optical Society of America, "for development of laser-manipulated quantum engineering at the single-atom level and application of these methods to quantum logic systems, atomic frequency standards, and fundamental tests of quantum mechanics." The award is the highest honor bestowed by the Optical Society of America, recognizing overall distinction in optics. Dr. Wineland was recognized for his group's world-leading research in quantum state engineering of trapped ions and repeated breakthroughs in quantum computing, precision frequency standards, and fundamental tests of quantum mechanics. These include the first demonstration of the Schrödinger cat superposition of states in a single ion, the first demonstration of entanglement of four ions, the first experimental demonstration of all the components required for a scalable quantum computing architecture, and many other firsts required for quantum computation. 


\section{I. Rabi Award}

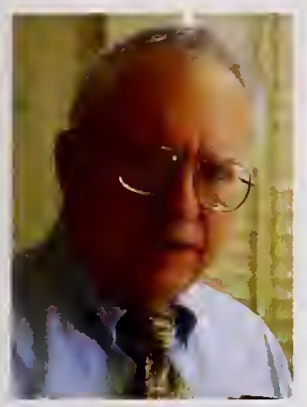

John L. Hall, Quantum Physics Division, received the 2004 I. I. Rabi Award of the IEEE Society for Ultrasonics, Ferroelectricity, and Frequency Control. Presented at the 2004 IEEE International Frequency Control Symposium, the award was for his long-term research and achievements in the area of optical frequency standards.

\section{Légion d'Honneur Award}

John L. Hall, Quantum Physics Division, was also selected for membership in 2004 in the Légion d'Honneur, the highest award that the Republic of France makes to foreigners with a distinction level of "Chevalier" ("Knight").

\section{E. Bright Wilson Award in Spectroscopy}

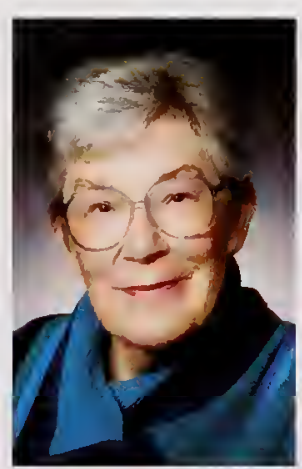

Marilyn E. Jacox, Scientist Emeritus, Optical Technology Division, received the 2003 E. Bright Wilson Award in Spectroscopy from the American Chemical Society for her outstanding contributions to fundamental spectroscopy of chemical reaction intermediates. She pioneered matrix isolation spectroscopy - a technique that involves snaring unstable molecules in an inert chemical framework so they can be studied-working from theory through novel experimental design to compilations of data on more than 3,400 neutral and ionic transient small molecules.

\section{Presidential Rank Award}

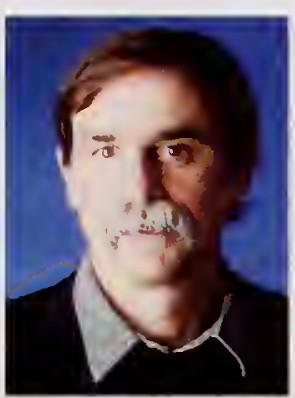

David J. Wineland, Time and Frequency Division, received a 2004 Presidential Rank Award for Distinguished Senior Executives and Professionals from the U.S. Office of Personnel Management for exceptional technical accomplishments, including development of optical frequency standards, fundamental tests of quantum mechanics, and research on quantum computing.

\section{Presidential Early Career Award}

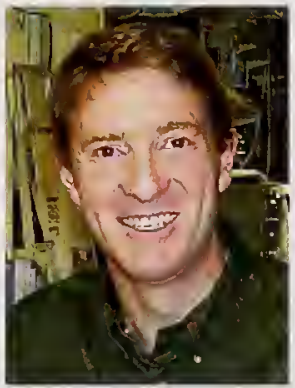

Scott A. Diddams, Time and Frequency Division, received a 2003 Presidential Early Career Award for Scientists and Engineers (PECASE) for leadership in developing and applying new laser techniques to measure the frequency of electromagnetic radiation to unprecedented accuracy and to support development of advanced standards for time and length. Dr. Diddams was recognized for his outstanding work on optical frequency synthesis based on femtosecond laser frequency combs. The PECASE award is the highest honor bestowed by the United States Government on scientists and engineers beginning their independent careers. Sixty awards were given nationally and presented in a White House ceremony. 


\section{Arthur S. Flemming Awards}

\section{Established in 1948, the Flemming Awards honor outstanding Federal employees with no more than fifteen years of government service. Recognized by the President of the United States, agency heads, and the private sector, about ten winners are selected each year from all areas of the Federal service.}

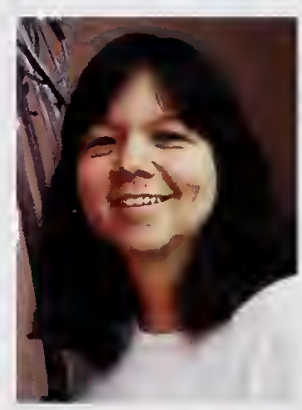

Deborah S. Jin, Quantum Physics Division, received a 2003 Flemming Award, for breakthrough achievements in coaxing collections of fermionic atoms into a single quantum state, analogous to the Bose-Einstein Condensate. In 1999, she produced the first degenerate Fermi gas of atoms in a tiny cloud of potassium atoms in a magnetic trap. In 2003 she created the first Bose-Einstein "Super Molecule," a Bose-Einstein Condensate of fermionic molecules.

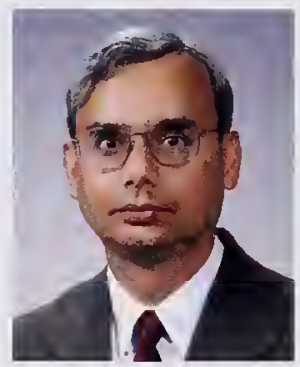

Muhammad Arif, Ionizing Radiation Division, received a 2002 Flemming Award for his accomplishments in the development of two major neutron research facilities and for major research accomplishments in the fields of neutron interferometry, neutron optics, and neutron imaging.

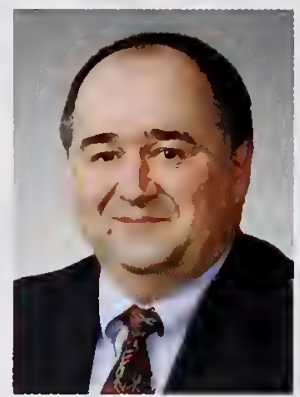

Marc F. Desrosiers, Ionizing Radiation Division, received a 2002 Flemming Award for developing and establishing alanine/EPR technology as the dosimeter of the highest metrological quality for measuring the absorbed dose from ionizing radiation beams used in radiation processing, and for extending its use and meeting the needs of industry through an innovative e-Certification system he developed for remote, internet-based, on-demand calibrations.

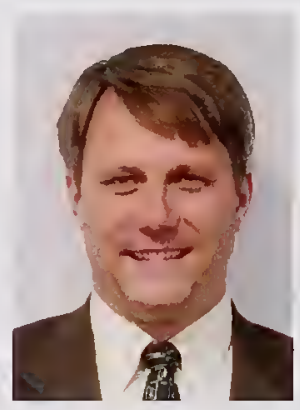

Mark D. Stiles,

Electron and Optical Physics Division, received a 2002 Flemming Award for his leadership in the exploration and exploitation of electronic and magnetic properties of nanometer-scale devices. His research, based on first-principles quantum mechanical calculations, has guided seminal experiments on electron flow in transistors, single-electron charging effects in tunnel junctions, spintronic devices, and magnetic multilayer structures. In particular, this body of work has had a significant impact on the understanding, development, and commercialization of "giant magnetoresistive" devices, a breakthrough technology for the data storage industry.

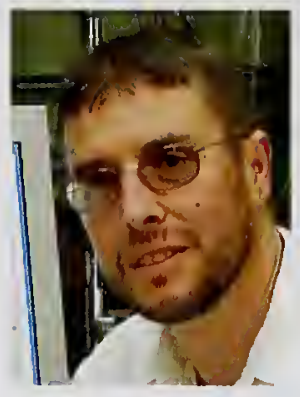

Charles S. Tarrio, Electron and Optical Physics Division, received a 2003 Flemming Award for pioneering contributions to the measurement of extreme ultraviolet (EUV) light, and its applications in semiconductor lithography, astronomy, and physical sciences. Dr. Tarrio established an EUV metrology facility at the NIST SURF synchrotron light source that has become the leading provider of measurement and characterization services to the international EUV optics community. During a decade of service this facility has performed well over 1000 tests for the user community, all on components that have been fabricated with tolerances at the cutting edge of measurement science. These include EUV telescopes that have been flown in satellite missions, optics for EUV semiconductor lithography systems, mirrors for $\mathrm{x}$-ray lasers, and a variety of new devices for the generation, control and measurement of EUV light. Since EUV light has a wavelength of 10 nanometers, its optics must be formed to an accuracy of 1 nanometer, the size of 10 atoms. His focus on accurate metrology has led Dr. Tarrio to make a number of discoveries that have advanced the nanometer-scale technology of EUV optics, ranging from basic optical properties of materials to improved methods of nanofabrication. 


\section{Fellowship of the American Association for}

\section{the Advancement of Science}

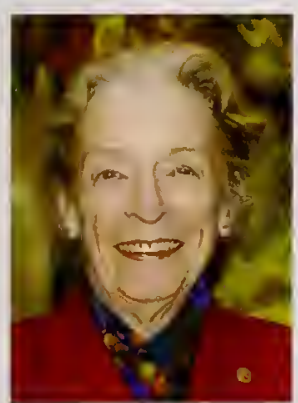

Katharine B. Gebbie, Director of the Physics Laboratory, was elected a 2004 Fellow of the American Association for the Advancement of Science "for her inspired directorship of the NIST Physics Laboratory, which is a world leader in the fields of atom cooling and trapping, nanotechnology, quantum metrology, and quantum computation."

\section{Fellowship of the American Physical Society}

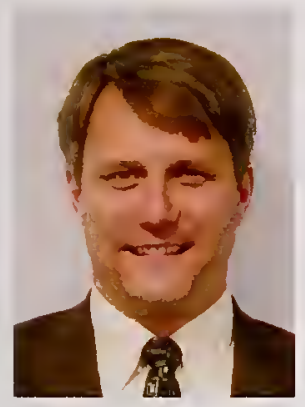

\author{
Mark D. Stiles, \\ Electron and \\ Optical Physics \\ Division, was elect- \\ ed a 2004 Fellow of \\ the American \\ Physical Society, \\ "for his creative and
} skillful use of first principles calculations and phenomenological models that have substantially contributed to our understanding of the physics of magnetic heterostructures."

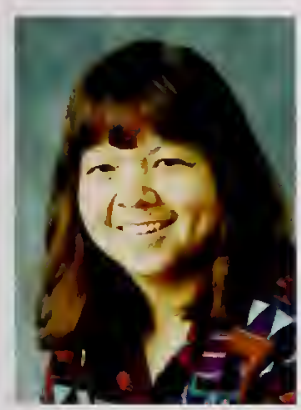

Deborah S. Jin, Quantum Physics Division, was elected a 2003 Fellow of the American Physical Society, for her innovative realization and exploration of a novel quantum system, the degenerate Fermi atomic gas.

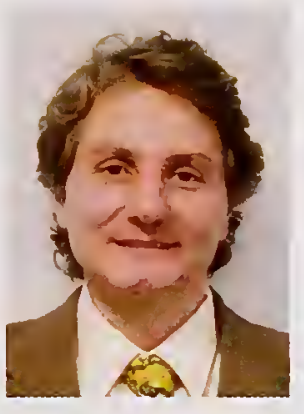

Joseph A. Stroscio, Electron and Optical Physics Division, was elected a 2002 Fellow of the American Physical Society, "for advancing our fundamental knowledge of semiconductor and metal surfaces and the innovative development, application, and dissemination of advanced methods of scanning tunneling microscopy and spectroscopy."
Washington Academy of Sciences Physical Science Award

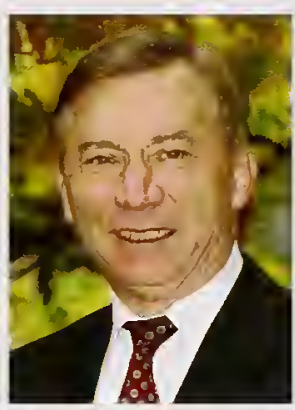

William R. Ott, Deputy Director of the Physics Laboratory, received the 2004 Physical Sciences Award from the Washington Academy of Sciences and was elected a Fellow for his leadership of the NIST Physics Laboratory in supporting industry, government, and the scientific community with measurement research and services in electronic, optical, and ionizing radiation technologies.

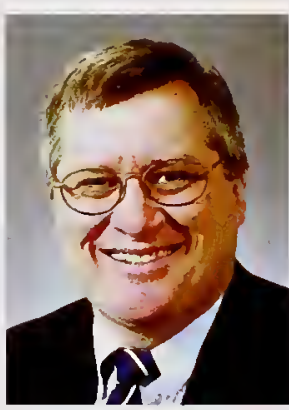

\section{Charles W. Clark} Electron and Optical Physics Division, received the 2003 Physical Sciences Award of the Washington Academy of Sciences, and was elected a Fellow for his contributions to the physics of quantum manybody systems.

\section{Fellowship of the Optical Society of America}

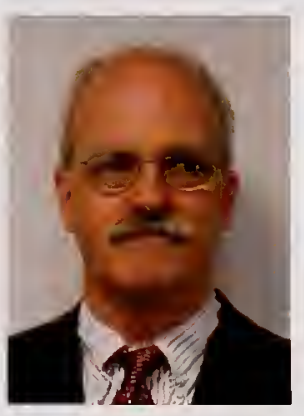

Jabez J. McClelland, Electron and Optical Physics Division, was elected a 2004 Fellow of the Optical Society of America, for contributions to atom optics, including the fabrication of stable structures by direct-write atomic lithography.

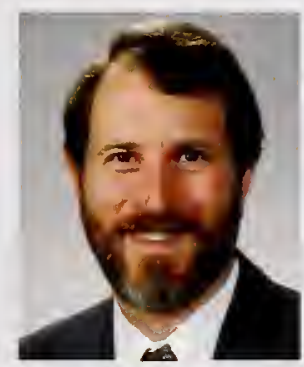

Paul D. Lett, Atomic Physics Division, was elected a 2003 Fellow of the Optical Society of America in recognition of his development of photoassociative spectroscopy as a tool for studying interactions between ultracold atoms. 


\section{Distinguished Visiting Fellow Award}

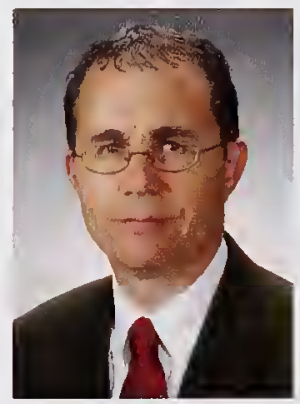

John D. Gillaspy, Atomic Physics Division, received the 2004 Distinguished Visiting Fellow Award at the new International Research Centre for Experimental Physics at the Queen's University of Belfast. He was recognized for his expertise in the physics and applications of highly charged ions, the focus of research in the "Ion Hall" that comprises most of one floor of the new Centre. The competitive award was designed to bring in internationally recognized physicists to enhance interdisciplinary research activity.

\section{Bourke Lectureship Award}

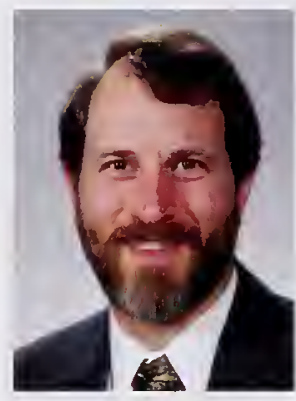

Paul D. Lett, Atomic Physics Division, received the 2004 Bourke Lectureship Award. He was recognized for his distinguished work on cooling and trapping of atoms, on ultralow energy atomic collisions and on photoassisted association at ultralow temperatures. Dr. Lett was selected by the Faraday Division of the Royal Society of Chemistry. The award was presented by the Imperial College, United Kingdom.

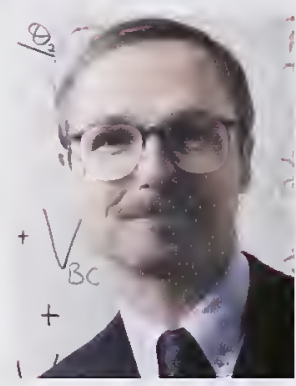

David J. Nesbitt, Quantum Physics Division, was awarded the 2002 Bourke Lectureship of the Faraday Division of the Royal Society of Chemistry. The Faraday Society instituted the lectureship in 1954 as a means to bring distinguished scientists from overseas to lecture in Britain.

\section{IEEE Ferroelectrics and Frequency Control Society}

\section{Distinguished Lecturer}

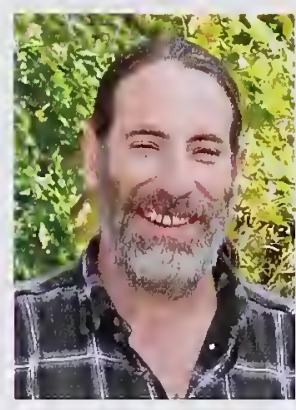

Steven Jefferts, Time and Frequency Division, was selected as the IEEE Ferroelectrics and Frequency Control Society Distinguished Lecturer for 2003-2004. Dr. Jefferts was selected because of his international prominence in the field of frequency standards as the leader of the NIST-F1 primary frequency standard team. Through the Distinguished Lecturer program, Dr. Jefferts gave many lectures on "Atomic Clocks-Past, Present, and Future" in Europe, Asia, and the United States.

\section{Tom L. Popejoy Dissertation Prize}

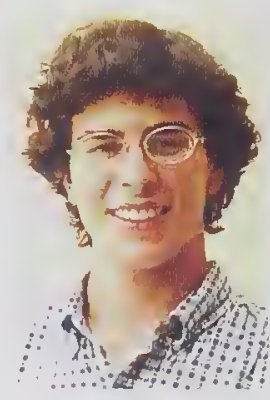

Joshua C. Bienfang, Electron and Optical Physics Division, received the 2003 Tom L. Popejoy Dissertation Prize of the University of New Mexico. Dr. Bienfang was recognized for his 2001 physics Ph.D. dissertation, Laser Frequency Stabilization and Nonlinear Conversion. The award citation describes this dissertation as "a rare combination of sophisticated experiments and theory."

\section{Sigma Xi (NIST Chapter) Young Scientist Award}

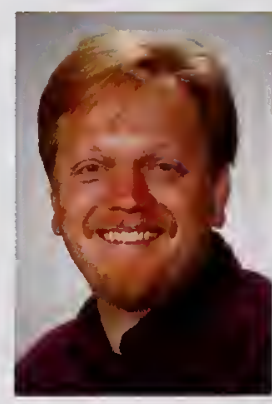

James Porto,

Atomic Physics Division, received the 2004 Young Scientist Award from the NIST Chapter of Sigma Xi. He was recognized for excellence in quantum engineering research using ion and atom traps.

\section{W. J. Youden Award}

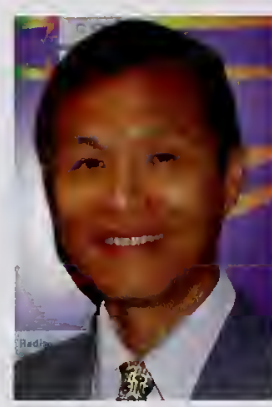

Kenneth G. W. Inn, lonizing Radiation Division, received the 2003 W. J. Youden Award in Interlaboratory Testing for recognition of the paper, An Alternative Statistical Approach for Interlaboratory Comparison Data Evaluation, published in the Journal of Radioanalytical and Nuclear Chemistry, 248, 163-173 (2001). 
James $F$.

\section{Gibbons Award}

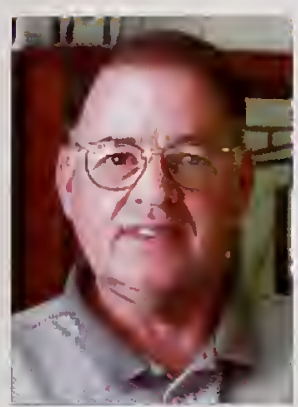

\section{David DeWitt,}

Faculty Appointment, Optical Technology Division, received the 2004 James F. Gibbons Award from the IEEE, for pioneering work in the field of advanced thermal processing of semiconductor materials.

\section{Best Paper Award in Applied Metrology}

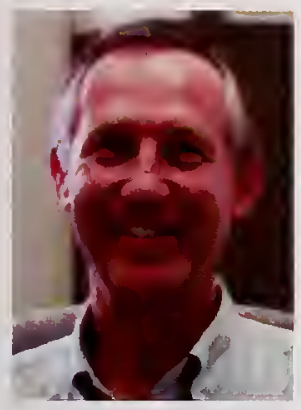

\section{George P. Eppeldauer, Optical Technology Division, received the Best Paper Award in the Applied Metrology Category of the} 2004 NCSL International Workshop and Symposium. The award was given for Irradiance Responsivity Scale Realization between $1 \mu \mathrm{m}$ and $2.5 \mu \mathrm{m}$, a paper describing the extension of the NIST reference detector responsivity scale from the visible to the infrared range. The paper also describes a newly developed extended-InGaAs radiometer that can propagate the new scale to calibrate infrared devices and systems with low measurement uncertainty.

\section{Gold Medal (DoC)}

The Gold Medal is the highest honor award conferred upon an employee by the Department of Commerce, for "distinguished performance characterized by extraordinary, notable, or prestigious contributions that impact the mission of the Department of Commerce andlor operating unit and which reflect favorably on the Department."

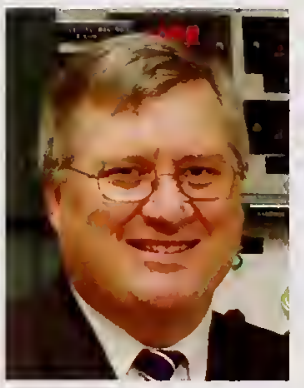

Charles W. Clark, Electron and Optical Physics Division, received a 2004 Gold Medal, "for leadership in establishing NIST as the world's foremost center of excellence in quantum computing and quantum information science and engineering."

Subsequent to major breakthroughs at NIST in quantum physics in 1995, Dr. Clark established a Quantum Information/Bose-Einstein Condensation center of excellence where scientists from around the world could work together on the latest ideas in quantum engineering. QIBEC has been immensely successful, due primarily to Dr. Clark's dynamic leadership. He created a forum for new developments in quantum communication, cryptography, and information processing, areas where NIST is now preeminent, and has promoted innovation and excellence through his leadership and personal technical excellence.

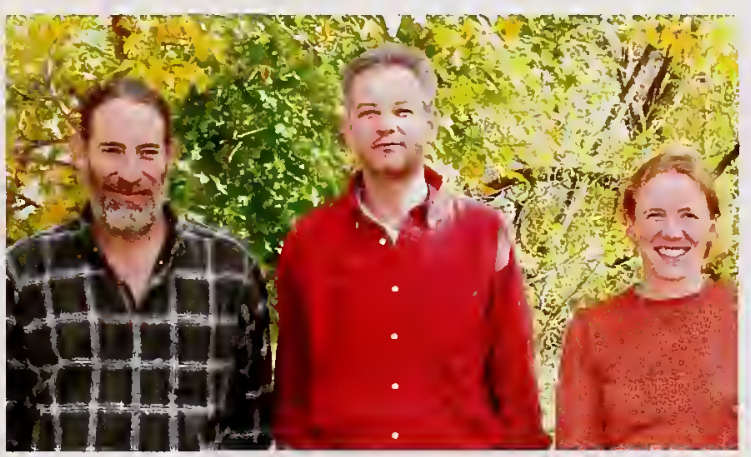

Steven Jefferts, Thomas Heavner, and Elizabeth Donley, Time and Frequency Division, received a 2004 Gold Medal for designing, constructing, operating, and continually improving the world's most accurate atomic clock, the NIST-F1 laser-cooled cesium fountain primary frequency standard. NIST-F1 is the U.S. national standard for frequency and the SI second, with a fractional frequency uncertainty of about $6 \times 10^{-16}$ as of December, 2004. NIST-F1 is the calibration source for the NIST time scale, which underpins NIST's most heavily used services, including the Internet Time Service and NIST radio broadcasts.

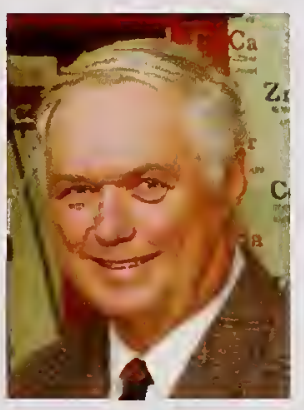

Albert C. Parr, Optical Technology Division, received a 2003 Gold Medal, "for strategic leadership in building major technical programs responsive to measurement needs of the Nation." These include the needs of the military for effective night vision sensors and missile detection systems and the needs of remote sensing networks for high accuracy optical radiation measurements to monitor global climate change. In addition, he addressed the needs of the microelectronics industry for noncontact optical probes of manufacturing processes and the needs of major manufacturing sectors for sophisticated color and appearance standards to ensure quality of consumer products. 


\section{Silver Medal (DoC)}

The Silver Metal is the second highest honor awarded by the Department of Commerce, for "exceptional performance characterized by noteworthy or superlative contributions that have a direct and lasting impact."

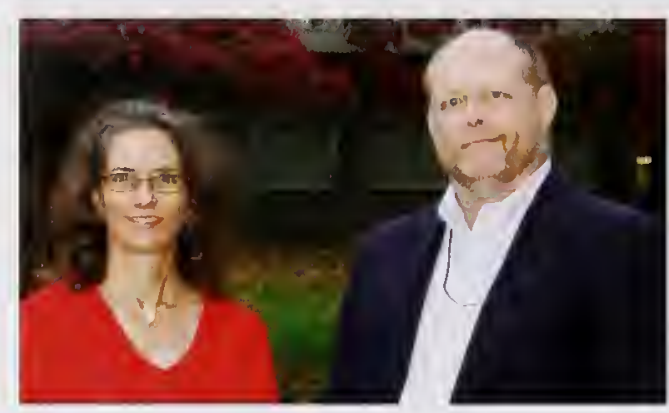

B. Carol Johnson and Steven W. Brown, Optical Technology Division, received a 2004 Silver Medal, "for their innovative approach to optical sensor calibration which resulted in a 6 percent decrease in the satellite-derived ocean chlorophyll a level, a critical measure of carbon dioxide intake by the oceans

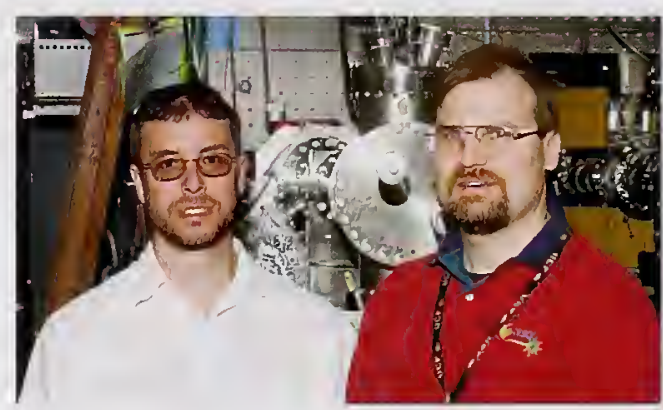

\section{Charles S. Tarrio and Steven Grantham,} Electron and Optical Physics Division, received a 2004 Silver Medal, "for establishing a measurement science program that has brought NIST to a position of world leadership in the emerging technology of extreme ultraviolet optics." Drs. Tarrio and Grantham combined basic measurement science with high responsiveness to customer needs, in an area of rapid technological change and key economic importance. They built a major national facility for the optical characterization of extreme ultraviolet (EUV) imaging systems based at the NIST Synchrotron Ultraviolet Research Facility (SURF), and established NIST as the clear international leader in EUV metrology. In light of their work, NIST has led the effort to standardize EUV optical measurements worldwide.

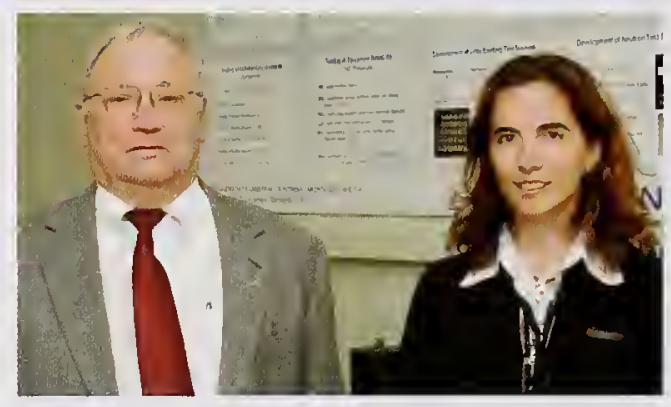

Michael P. Unterweger Jr. and Leticia Pibida, Ionizing Radiation Division, received a 2004 Silver Medal for leadership in the development and implementation of radiation detector standards for homeland security. These standards address the performance of radiological detection equipment to be used by personnel involved in border control, customs, coast guard, and postal services requiring the detection, monitoring, and interdiction of illicit radiological material entering and transported through the country. These standards will provide crucial support for first responders needing reliable measurements of radioactive material in homeland security situations.

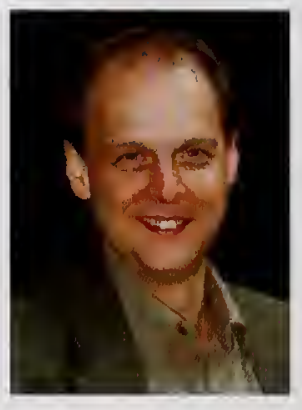

Carl J. Williams, Atomic Physics Division, received a 2003 Silver Medal, for leadership of the NIST Quantum Information Program. The

Program combines NIST laboratory research efforts in Boulder, $\mathrm{CO}$, and Gaithersburg, MD, in a coordinated effort towards early implementation of quantum information processing technology. It has grown into a multidisciplinary effort that is internationally recognized for its accomplishments, and is a primary provider of quantum information technology and expertise to the defense, intelligence, and scientific communities. 


\section{Bronze Medal (NIST)}

The Bronze Medal is the highest honorary recognition available for Institute presentation. The award recognizes work that has resulted in more effective and efficient management systems, as well as the demonstration of unusual initiative or creative ability in the development and improvement of methods and procedures. It is also given for significant contributions affecting major programs, scientific accomplishment within the Institute, and superior performance of assigned tasks for at least five consecutive years.

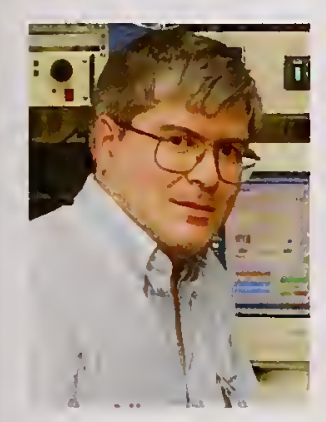

Michael G. Mitch, Ionizing Radiation Division, received a 2004 Bronze Medal for providing measurement services to enable the consistent, safe, and efficacious use of brachytherapy radiation sources, such as prostate seeds. The implantation of tiny radioactive seeds in the prostate to kill diseased tissue is being increasingly used for cancer therapy. This has stimulated a ten-fold growth in manufacturers of such seeds. Since 1999 , when NIST introduced a primary standard for measuring seed strength with high accuracy regardless of design, Dr. Mitch worked with manufacturers, secondary calibration labs, and clinics to disseminate this unique capability. His services are important for ensuring stability and consistency of manufacturing processes, necessary for FDA and NRC approval and the eventual use of the seeds in patients. He works closely with a national network of accredited calibration labs in transferring seed calibrations and providing proficiency testing.
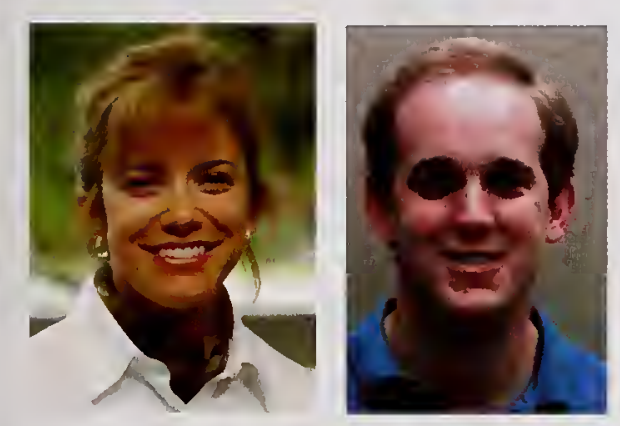

Maria E. Nadal and Edward A. Early,

Optical Technology Division, received a 2004

Bronze Medal for developing a state-of-the-art instrument to meet industry's needs for improved color measurements and standards. Incorrect color measurements waste $2 \%$ to $3 \%$ of manufacturing costs and affect virtually every commercial product and all tiers of the supply chain due to the wide use of plastics, paints, coatings, inks, pigments, and dyes by manufacturers. The team also developed the statistical analysis tools to help industry determine their color measurement uncertainties. By allowing U.S. products to meet stringent color specifications, independent of manufacturing locale, these new NIST capabilities and measurement services have strengthened U.S. industrial comperitiveness.

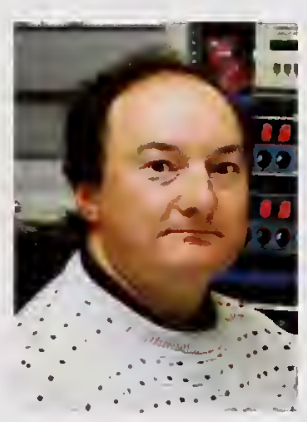

Joseph P. Rice, Optical Technology Division, received a 2004 Bronze Medal for developing an innovative radiometer to link infrared measurements from U.S. satellites to NIST optical radiation scales. Satellite sensors provide information about land and sea-surface temperatures, cloud heights, humidity profiles, concentration and distributions of atmospheric molecules and pollutants, and forest fires. 


\section{Bronze Medal (NIST) (continued)}

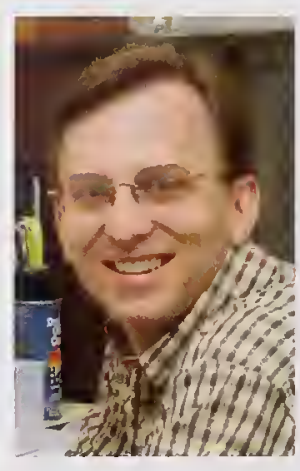

Robert E. Vest, Electron and Optical Physics Division, received a 2004 Bronze Medal for providing high quality calibration services for far ultraviolet detectors and working with customers to improve the measurement technology. Photodetectors calibrated on his beamline at the NIST Synchrotron Ultraviolet Radiation Facility are found in virtually every major UV radiation measurement laboratory in the world. With his collaborators, he has devised several new detectors for applications of growing industrial importance, such as $193 \mathrm{~nm}$ and $157 \mathrm{~nm}$ lithography.

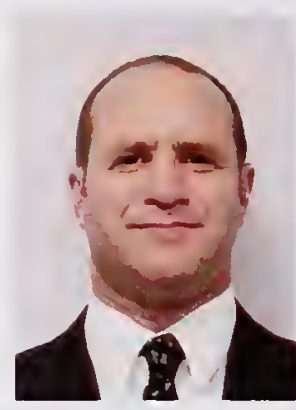

Alan H. Band, Electron and Optical Physics Division, received a 2003 Bronze Medal for his outstanding success designing and fabricating electronic instrumentation. Mr. Band designed and supervised the construction of the Electron Physics Group's scanning tunneling microscope, autonomous atom assembler, scanning electron microscope with polarization analysis, and single atom-ondemand source. Mr. Band has frequently been sought out for collaborative projects with other Laboratories within NIST. Because of his skill, industry, and creativity, he was instrumental in the success of several highly important NIST programs. In addition, the Smithsonian currently features a display he designed for their "Science in American Life" exhibit.

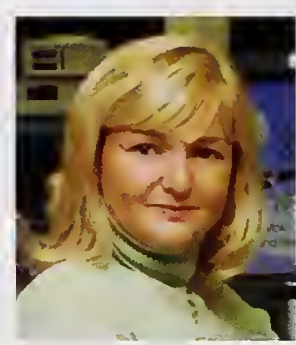

Angela R. Hight Walker, Optical Technology Division, received a 2003 Bronze Medal, for leadership in developing stronger programmatic ties between NIST and NIH and in increasing the opportunities for scientific collaboration and institutional cooperation with $\mathrm{NIH}$.

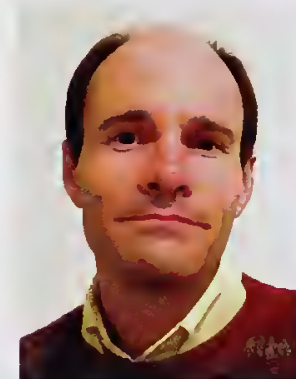

Lawrence T. Hudson, Ionizing Radiation Division, received a 2003 Bronze Medal "for outstanding response to a national emergency by coordinating and implementing a protocol for the decontamination of the mail by irradiation." His design and validation of an $x$-ray imaging process permitted parcels that had been quarantined to be sanitized and released after the widely publicized anthrax terror attacks using the U.S. mail system.
Samuel Wesley Stratton Award (NIST)

\author{
The Samuel Wesley Stratton Award \\ is granted for outstanding scientific \\ or engineering achievements in \\ support of NIST objectives.
}

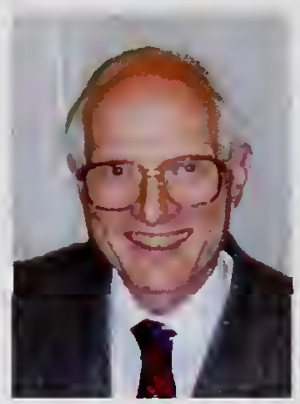

Paul S. Julienne, Atomic Physics Division, received the 2004 Stratton Award for worldleading theoretical physics research in ultracold collisions fundamental to the laser cooling of atoms and to Bose-Einstein condensation.

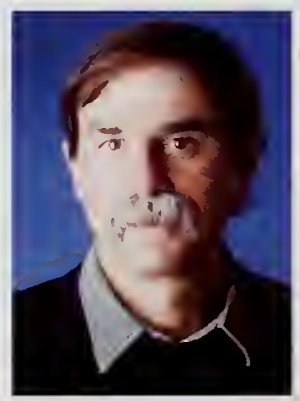

David J. Wineland, Time and Frequency Division, received the 2003 Stratton Award for pioneering research in quantum engineering for quantum computing, precision frequency standards, and fundamental tests of quantum mechanics. Dr. Wineland was recognized for his Ion Storage Group's world-leading research in quantum state engineering of trapped ions with repeated breakthroughs in quantum computing, precision frequency standards, and fundamental tests of quantum mechanics. 


\section{Key to Table}

\section{Abbreviations}

STRS Congressionally appropriated funds for NIST's Scientific and Technical Research and Services

ATP Intramural research funds provided to support the goals of the NIST Advanced Technology Program

OA Funds provided by other agencies in support of needed research and measurement services

Other Other sources of funding, including calibration fees

\section{Federal Agencies Supporting} Physics Laboratory Research

- Department of Commerce

- Department of Defense

- Department of Energy

- Department of Health and Human Services

- Department of Homeland Security

- Department of Justice

- Environmental Protection Agency

- National Aeronautics and Space Administration

- National Science Foundation

\begin{tabular}{|c|c|c|c|c|c|}
\hline \multicolumn{6}{|c|}{$\begin{array}{l}\text { PHYSICS LABORATORY RESOURCES } 2000-2004 \\
\text { (\$MLLLIONS) }\end{array}$} \\
\hline & 2000 & 2001 & 2002 & 2003 & 2004 \\
\hline STRS & 34.8 & 37.1 & 39.9 & 43.1 & 40.3 \\
\hline ATP & 1.8 & 2.1 & 2.9 & 2.7 & 0.6 \\
\hline$O A$ & 10.6 & 11.9 & 13.6 & 14.0 & 20.8 \\
\hline Other & 4.4 & 4.5 & 5.0 & 5.2 & 5.2 \\
\hline TOTAL & 51.6 & 55.6 & 61.4 & 65.0 & 66.9 \\
\hline
\end{tabular}

\section{Representative Private Sector Collaborators}

- American Association of Physicists in Medicine

- American National Standards Institute

- American Physical Society

- American Society for Testing and Materials

- Commission Internationale de l'Éclairage

- Council for Optical Radiation Measurements

- Council on Ionizing Radiation Measurements and Standards

- Electric Power Research Institute

- EUV LLC

- Illuminating Engineering Society of North America
- Institute of Electrical and Electronics Engineers

- International Electrotechnical Commission

- International Organization for Standardization

- National Council on Radiation Protection and Measurements

- NCSL International

- Nuclear Energy Institute

- Optical Society of America

- Optoelectronics Industry Development Association

- International SEMATECH

- SAE International

- SPIE-The International Society for Optical Engineering 
PHYSICS LABORATORY

Katharine Gebbie, Director - William Ott, Deputy Director

Electron and Optical Physics Division

Charles Clark, Chief

Atomic Physics Division

Carl Williams, Chief

Optical Technology Division

Albert Parr, Chief

Lisa Karam, Acting Chief

Time and Frequency Division

Thomas O'Brian, Chief

Quantum Physics Division

Steven Cundiff, Chief 


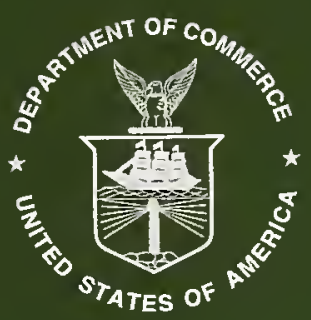

EDITORS:

Jonathan E. Hardis and William R. Ott

Physics Laboratory, National Institute of Standards and Technology

U.S. Department of Commerce Donald L. Evans, Secretary

Technology Administration Phillip J. Bond, Under Secretary for Technology

National Institute of Standards and Technology Hratch G. Semerjian, Acting Director

NIST SP 1033

February 2005

Disclaimer: any mention of commercial products is for information only; it does not imply NIST recommendation or endorsement, nor does it imply that the products mentioned are necessarily the best available for the purpose. 
NIST PHYSICS LABORATORY

100 Bureau Drive, Stop 8400

Gaithersburg, MD 20899-8400

wuw@physics.nist.gov 